\title{
ZT-P: An Advanced Air Core Reversed Field Pinch Prototype
}

Kurt F. Schoenberg
C. J. Bucheriauer
L. C. Burkhardt
L. D. Caudill
R. S. Dike*
T. Dominguez
J. N. Downing
P. R. Forman
J. A. Garcia
A. J. Giger
R. F Gribble
A. Haberstich
C. F. Hammer
L. J. Iko
J. C. Ingraham
R. W. Kewish

P. L. Klingner

T. W. Liniton**

E. M. Little ${ }^{\dagger}$

J. G. Melton

T. L. Petersen

W. A. Reass

N. L. Salazar

J.D. Sandoval

J. S. Sandovai

A. E. Schofield

J. D. Smith

E. K. Spanos

P. G. Weber

R. W. Wilkins

G. A. Wurden

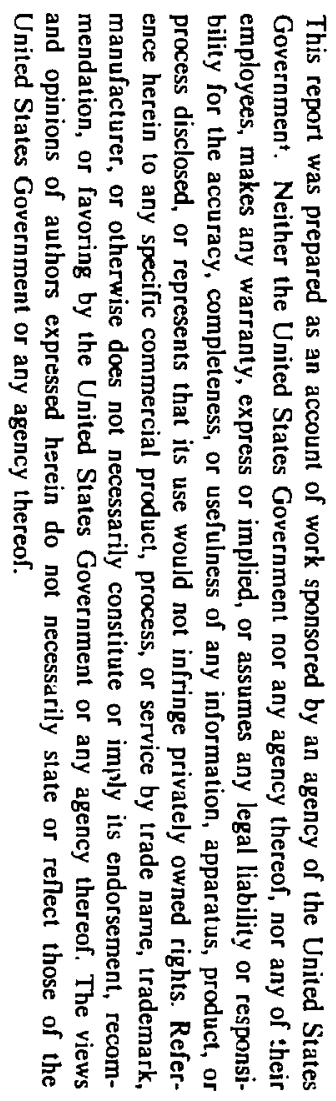




\section{ZT-P: AN ADVANCED AIR CORE REVERSED FIELD PINCH PROTOTYPE}

by

Kurt F. Schoenberg, C. J. Buchenauer, L. C. Burkhardt, L. D. Caudill, R. S. Dike, T. Dominguez, J. N. Downing, P. R. Forlian, J. A. Garcia, A. J. Giger, F. F. Gribble, A. Haberstich, C. F. Hammer, L. J. Iko, J. C. Ingraham, R. W. Kewish, P. L. Klingner, T. W. Linton, E. M. Little, J. G. Melton, T. L. Petersen, W. A. Reass, N. L. Salazar, J. D. Sandoval, J. S. Sandoval, A. E. Schofield, J. D. Smith, E. K. Spanos, P. G. Weber, R. W. Wilkins, and G. A. Wurden.

\section{ABSTRACT}

The ZT-P experiment, with a major radius of $0.45 \mathrm{~m}$ and a minor radius of $0.07 \mathrm{~m}$, was designed to prototype the next generation of reversea field pinch (RFP) machines at Los Alamos. ZT-P utilizes an air-core poloidal field system, with precisely wound and positioned rigid copper coils, to drive the plasma current and provide plasma equilibrium with intrinsically low magnetic field errors. ZT-P's compact configuration is adaptable to test various first wall and limiter designs at reactor-relevant current densities in the range of 5-20 MA/ $\mathrm{m}^{2}$. In addition, the load assembly design allows for the installation of torcidal field divertors. Design of ZT-P began in October 1983 and assembly was completed in octooer 1984. This report describes the magnetic, electrical, mechanical, vacuum, diagnostic, data acquisition and control aspects of the machine design. In addition, preliminary data from initial ZT-P operation are presented. Because of $2 T-P^{\prime}$ s prototypical function, many of its design aspects and experimental results are directly applicable to the design of a next generation RFP.

\section{INTRODUCTION}

The reversed field pinch (RFP) is an axisymmetric toroidal confinement device in which plasma is confined by a poloidal magnetic field generated by toroidal plasma current, and a toroidal magnetic field generated by poloidal plasma current and external windings. In contrast to the Tokamak, the mean toroidal and poloidal magnetic fields in the RFP plasma are of comparable 
strength resulting in high magnetic shear. In addition, the toroidal field at the plasma edge is weak compared to the poloidal field, and is reversed with respect to its direction on the magnetic axis. A sketch of RFP field topology is shown in Fig. I.1.

The internal RFP magnetic fields are largely generated by currents flowing within the plasma, and hence good confinement requires only modest fields at the magnet coils. That is, the engineering beta, defined as the ratio of the average plasma pressure to the magnetic field pressure at the coils can be high, allowing energetic plasmas to be confined by external coils of modest size and current. These confinement advantages esta sh the RFP as a strong candidate for a compact ohmically-heated fusion reactor.1

The promising advances in plasma performance, seen in the present generation of RFP experiments, have enhanced the RFP's reactor potential. However, design and operating limitations in present devices have clearly indicated the necessity for an advanced, next generation experiment to continue studies of RFP confinement at higher reactor-relevant currents and temperatures. The ZT-P experiment was designed to prototype such an advanced next-generation device.

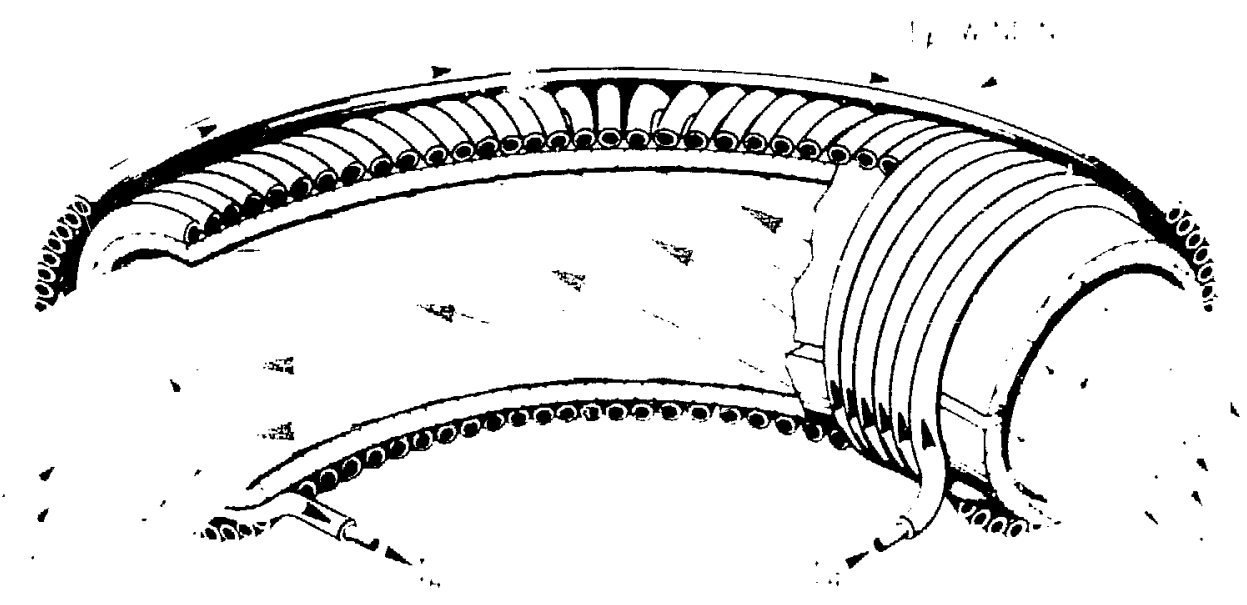

Fig. I. 1.

A conceptual representation of RFP magnetic field topology. 
A sumnary of the ZT-P operating parameters is listed in Table 1 . ZT-P utilizes an air-core poloidal field system with precisely wound and positioned rigid copper coils to drive the plasma current and provide plasma equilibrium with intrinsically low magnetic field errors. ZT-P also incorporates a modular design to facilitate assembly and disassembly, allowing for rapid replacement of various machine components. A photograph of the ZT-P experiment is shown in Fig. I.2.

\section{TABLE 1}

\section{ZT-P Parameters}

$\begin{array}{lc}\text { Minor Radius (m) } & 0.07 \\ \text { Major Radius (m) } & 0.45 \\ \text { Design Curtent (kA) } & 300 \\ \text { Design Current Density }\left(\mathrm{MA} / \mathrm{m}^{2}\right) & 20 \\ \text { Design Current Duration (ms) } & 3-5 \\ \text { Design Field Error Spec }\left(\delta_{\mathrm{B}} / \mathrm{B}_{\Theta}(\mathrm{a})\right) & 0.003 \\ \text { Magnetizing Coil Volt Seconds (Unipolar) } & 0.4 \\ \text { Magnetizing Coil Ampere-Turns } & 2.3 \times 10^{6} \\ \text { Poloidal Field System Energy (MJ) } & 1\end{array}$

The primary mission of ZT-P is to aid in the engineering design of future high current RFP devices, and to complement the RFP physics research presently under way on existing experiments. ZT-P will provide a test bed for many engineering aspects of large air-core machine design. These include, but are not limited to, plasma formation and equilibrium control with an air-core system, performance and reliability of the air-core poloidal and toroidal field circuits, and data acquisition and experimental control in the presence of high ambient magnetic fields and transient voltages. 


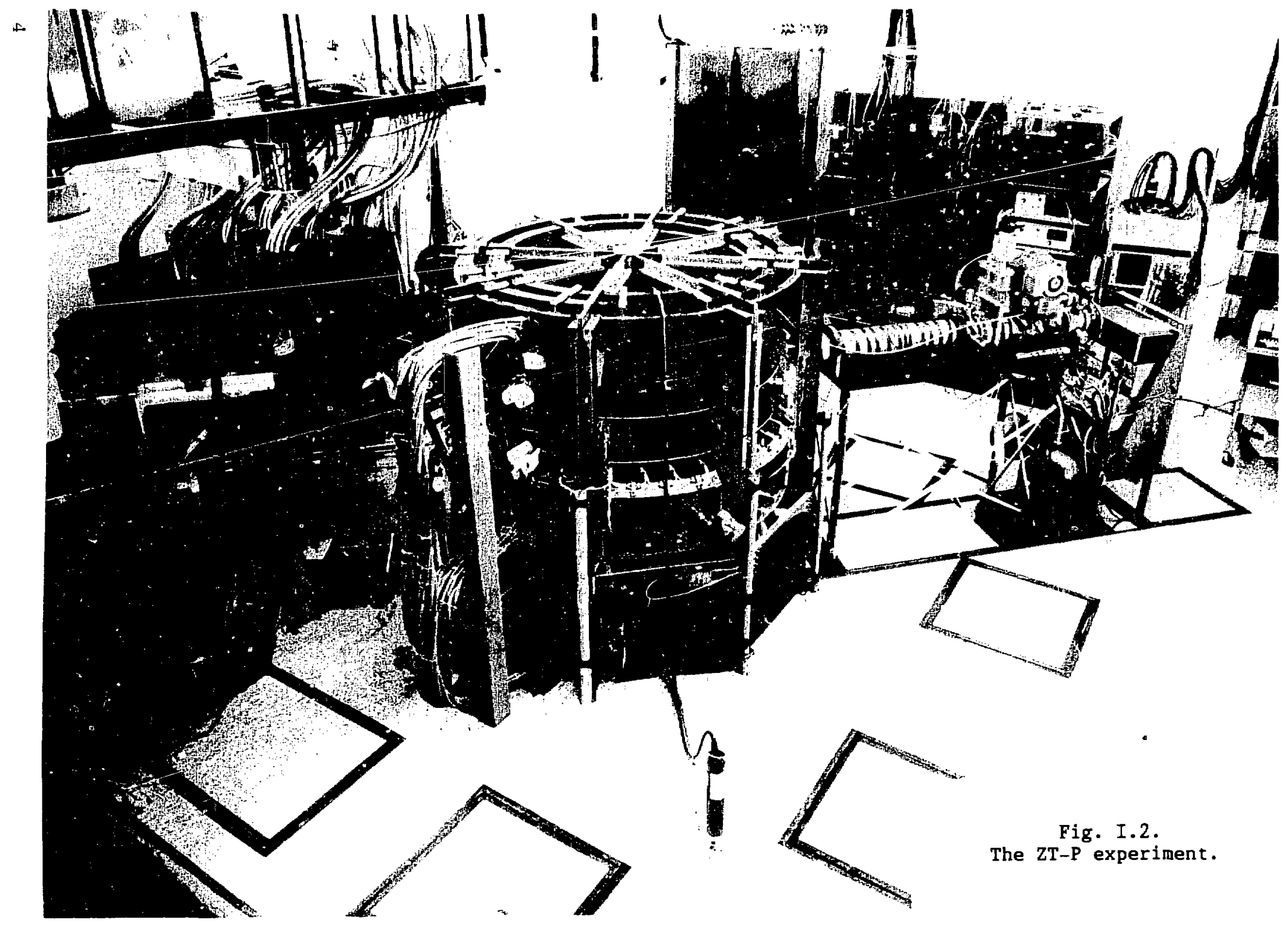


Determining the effect of magnetic field errors on RFP plasma confinement and the role of a conducting shell on RFP stability is essential to understanding and quantifying RFP performance. ZT-P is, therefore, designed for low error field operation and fast equilibrium control in the absence of a conducting shell. ZT-P's compact configuration is also adaptable to test various first wall and limiter designs at reactor-relevant current densities in the range of 10 to $20 \mathrm{MA} / \mathrm{m}^{2}$, and the load assembly design allows for the installation of toroidal field divertors.

Design of ZT-P began in 0ctober 1983 and assembly was completed in 0ctober 1984. This report describes the magnetic, electrical, mechanical, vacuum, diagnostic, data acquisition, and control aspects of the machine design. In addition, some preliminary data from initial ZT-P operation are presented. Because of ZT-P's prototypical function, many of its design aspects and experimental results are directly applicable to the design of a next generation RFP.

\section{MAGNETICS DESIGN}

\section{Poloidal Field System}

The poloidal field system in an RFP serves two important functions. It induces the toroidal electric field that drives the plasma current over the duration of the discharge, and it provides the magnetic boundary conditions necessary for plasma equilibrium. In ZT-P this functional dichotomy is achieved by using two separate systems of coils. The equilibrium coils provide the vertical field and field index necessary for plasma equilibrium, and the magnetizing coils provide the flux swing necessary to set up and maintain the discharge. A cross-sectional drawing of the poloidal field system is shown in Fig. II.1. 

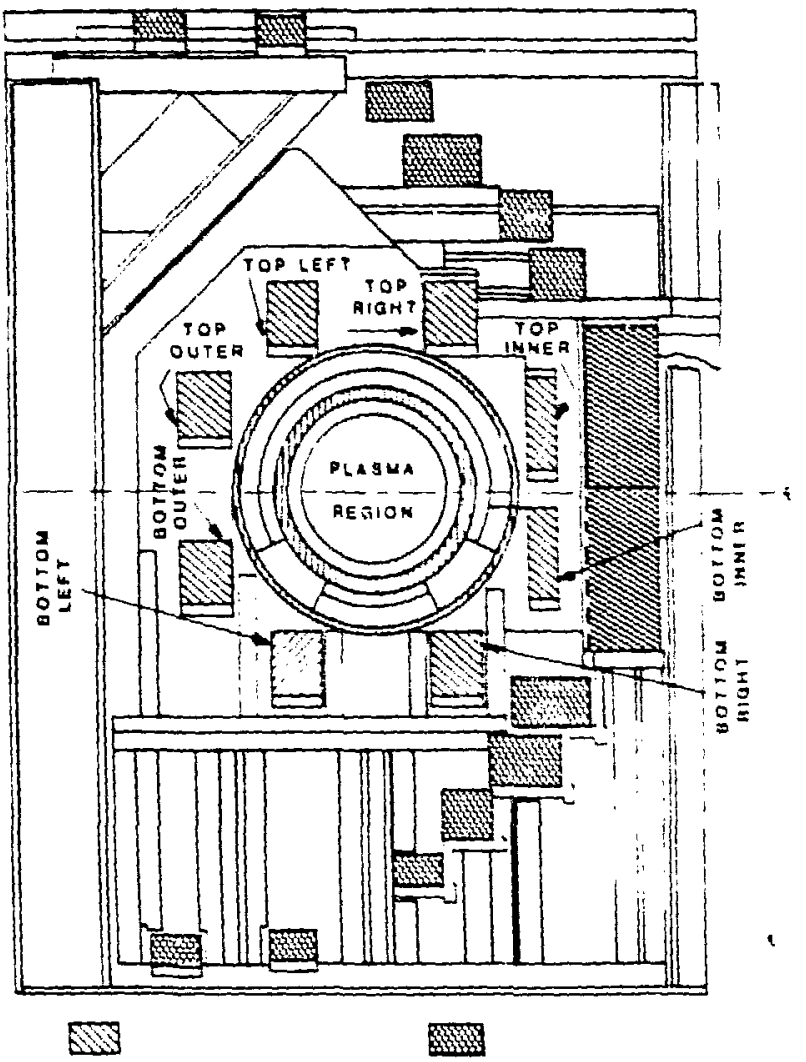

EQUILIBRIUM COHS MAGNETIZING COILS

Fig. II.I.

Cross-sectional representation of the ZT-P poloidal field system.

The main motivation behind the two coil system resides in the ability to inductively decouple the magnetizing and equilibrium coils. In principle, the decoupling greatly zimplifies plasma equilibrium control since the equilibrium coil current and corresponding equilibrium vertical field are inductively driven by the plasma, and ience proportional to the plasma current. Corrections to the vertical field for fine tuning of the plasma equilibrium may then be accomplished by using trimming supplies. Thus, the experimental control of plasma curient and plasma equilibrium are separable functions. Physically, the coil decoupling requires all magnetizing flux to completely link the equilibrium coil and plasmi. That is, all magnetizing flux is spatially confined outside the equilibrium coil cage. This constraint also insures the minimization of stray magnetic fields and concomitant field errors in the plasma region.

The magnetizing coil is composed of two sets of windings with 40 turns each. The windings are symmetrically arranged with respect to the machine mid-plane to allow series or parallel connection. The winding configuration incorporates design constraints to maximize physical access to the torus, maximize electrical coupling efficiency between the magnetizing winding and the plasma, and minimize axisymmetric field errors in the plasma due to flux leakage. Numerical programs using elliptic integrals were composed to calculate fields, flux surfaces, inductances, coil forces, and coil positions for minimum magnetic error field within the plasma under specified 
constraints. After several sets of iterations, in which the energy coupling efficiency was calculated using the SCEPTRE circuit code and a coupled RFP 0-D model,$^{2,3}$ a coupling eificiency of $12 \%$ was obtained.

The equilibrium winding is responsible for maintaining plasma equilibrium over the duration of the discharge. The equilibrium winding must therefore provide a time-varying vertical field with a proper field index that tracks the plasma equilibrium. ${ }^{4}$ In addition, the equilibrium winding must also satisfy design constraints to maximize physical access to the torus for maintenance, vacuum pumping, and plasma diagnostics; minimize the applied power necessary to control the equilibrium field; and minimize axisymmetric field errors in the discharge chamber.

A cross-sectional diagram of the equilibrium coil system is shown in Fig. II.2. The system consists of eight discrete coils that are each composed of multiple turns and are symmetric about the equatorial plane. As previously noted, most of the equilibrium vertical field is inductively driven by the plasma. However, lecause the spacing between the equilibrium coil and the discharge chamber is large, this inductive drive provides only $70 \%$ of the required equilibrium field during the $400-\mu \mathrm{s}$ plasma current rise. As a

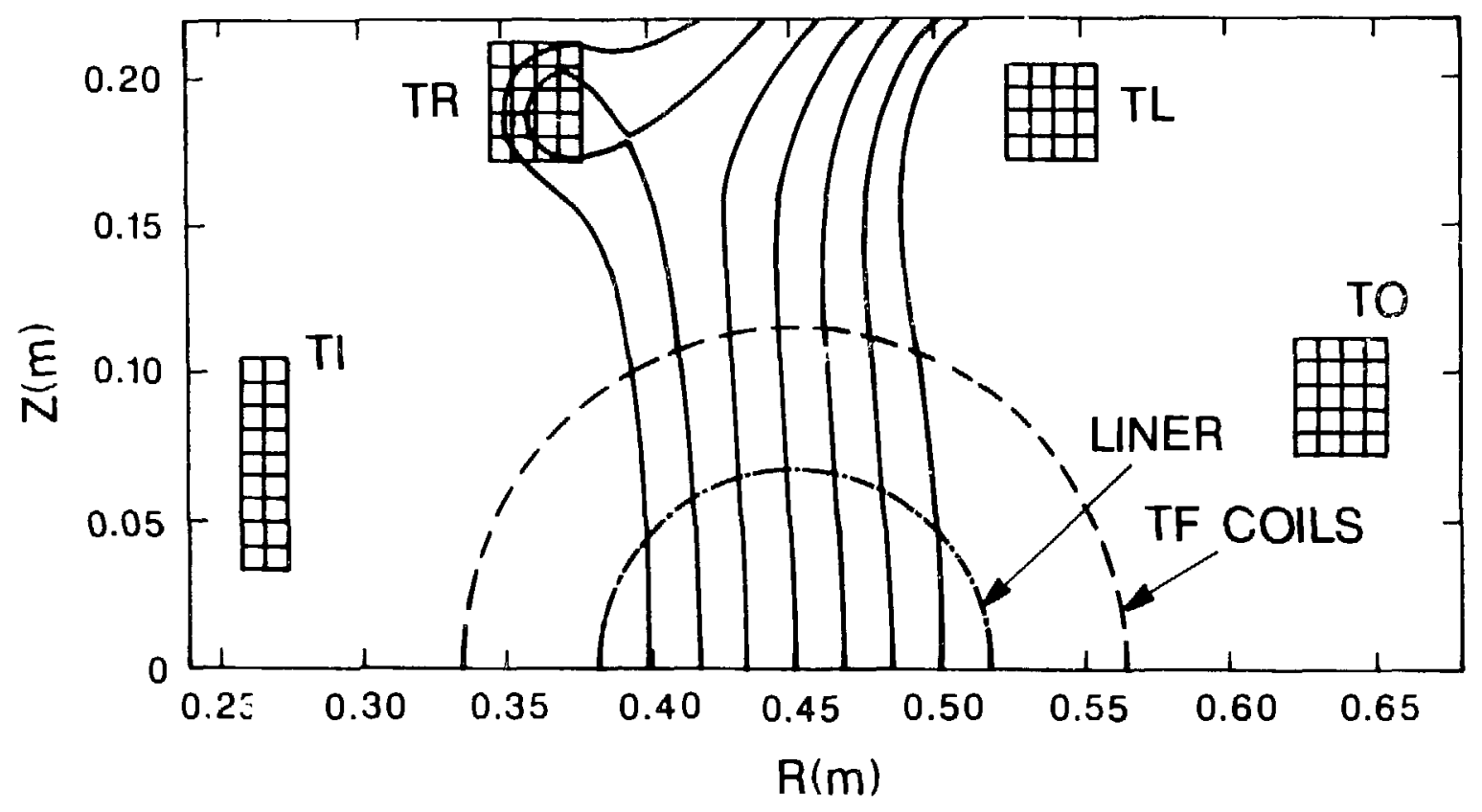

Fig. II. 2 .

Equilibrium coil cross section and vacuum vertical field plot. 
result, the equilibrium coils are configured to aid the inductive drive by utilizing some of the magnetizing flux. In this way, the power required to track plasma equilibrium during the current rise and during flat-topped operation without a conducting shell is maintained at an acceptable level. Figure II.3 illustrates the equilibrium coil cross-connection. Computer simulations have shown that the correct vertical field and field index can be achieved with the use of only two external power supplies if this coil crossconnection is used.

The equilibrium properties of the poloidal field system were checked by a code that numerically solves the Grad-Shafranov equation in the presence of fixed, current-carrying conductors. Code input required the spatial location of each magnetizing and equilibrium coil, the total current in each coil, and a poloidal flux function and pressure profile representative of the predicted ZT-P performance. ${ }^{4}$ The code results are illustrated in Fig. II.4. The computations indicate that winding controlled equilibria are physically viable for the proposed poloidal field system. The equilibrium coils are capable of controlling the plasma equilibrium over the range of machine operation, while

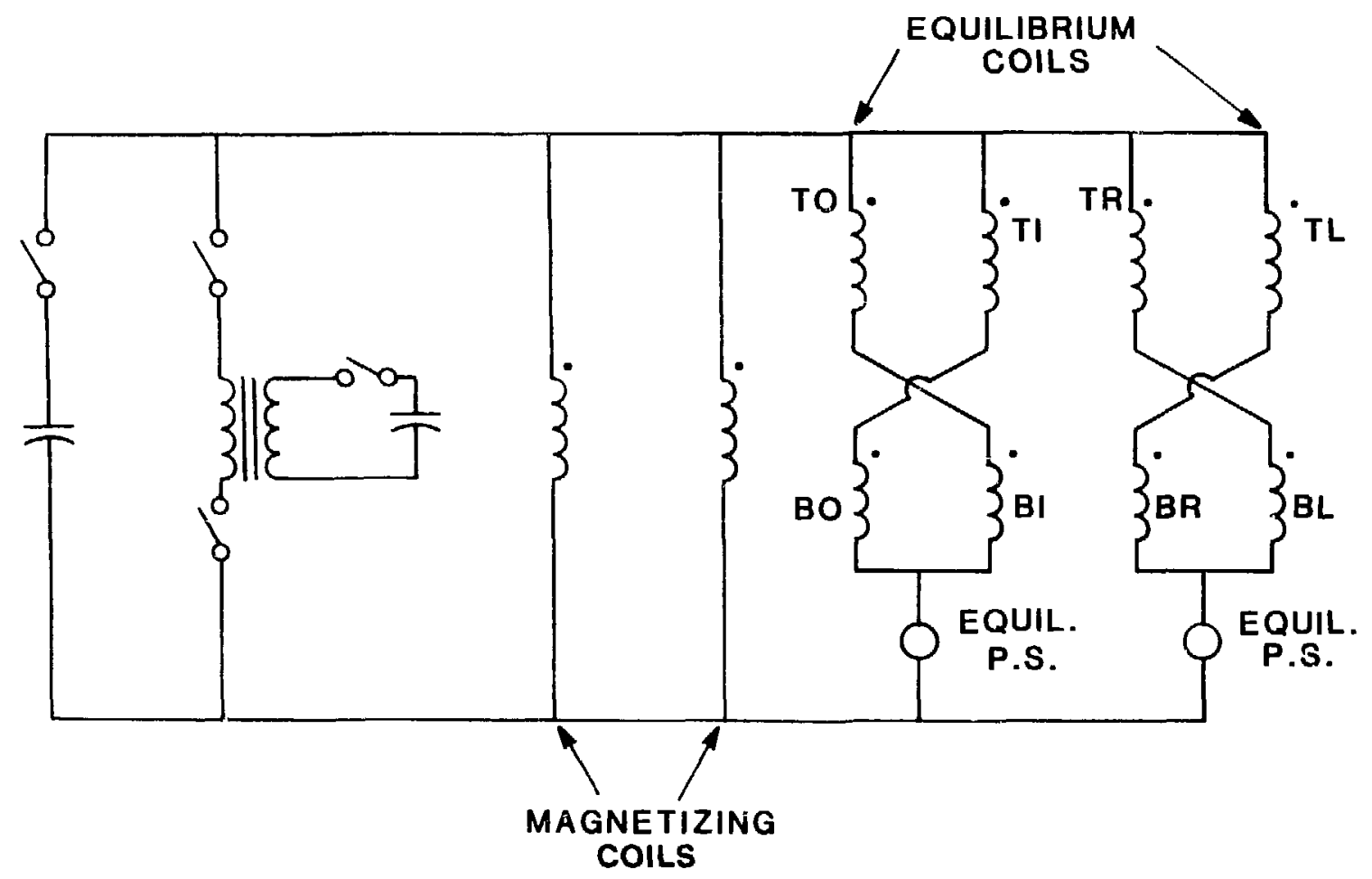

Fig. II.3.

Poloidal field coil connections. 


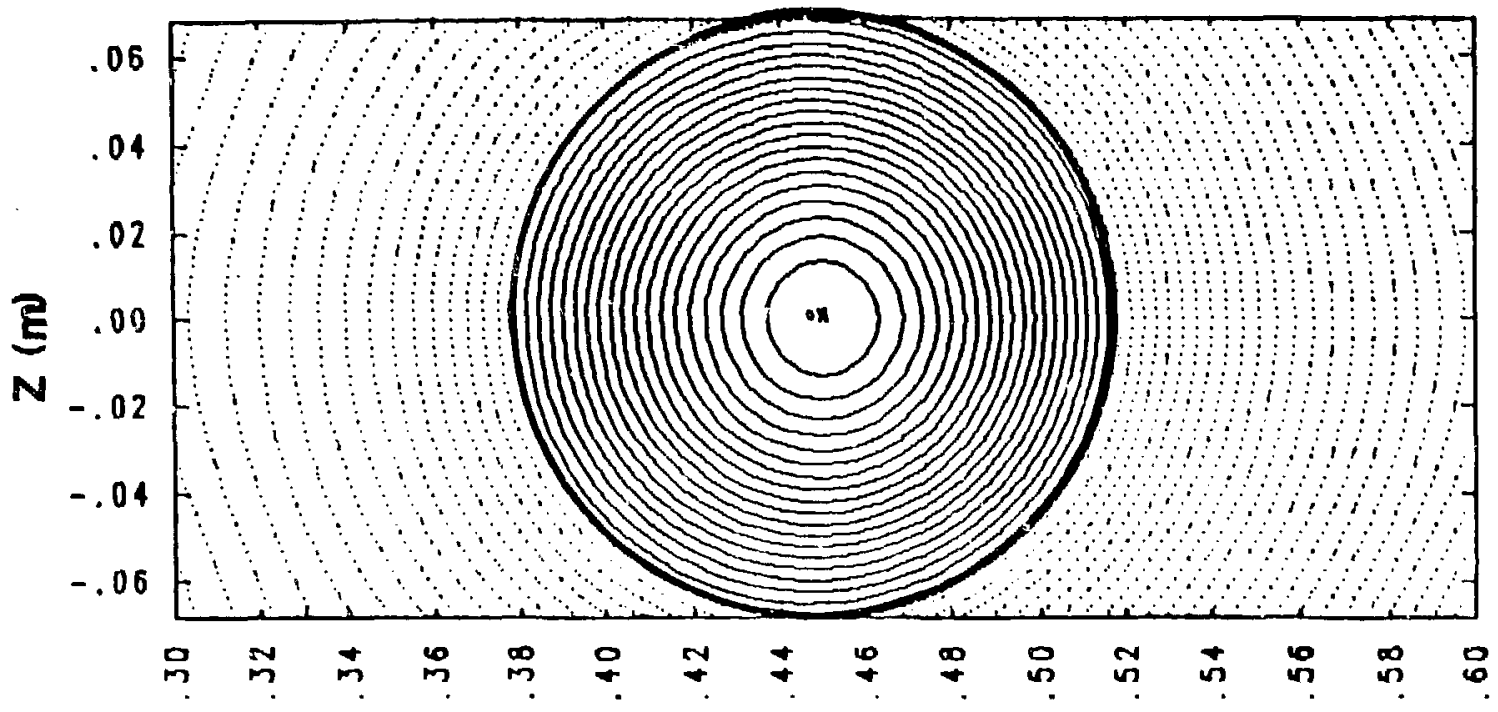

\section{MAJOR RADIUS (m)}

Fig. II.4.

ZT-P poloidal flux plot. The $(\cdot, x)$ denote the positions of the (geomètric, magnetic) axes, respectively.

the total flux surface distortion due to the discrete magnetizing and equilibrium coils is less than $4 \%$ of the minor plasma radius.

Toroidal Field System

Because an RFP operates at a lower value of toroidal magnetic field than a comparable Tokamak for the same plasma current, RFP design is not limited by mechanical stresses on the coils. An important design consideration, however, is the magnetic field errors induced in the plasma region due to field ripple and current feeds. It is hypothesized that asymetric field errors can deleteriously affect RFP discharge performance by both increasing plasma wall interactions in the vicinity of the error, thereby increasing plasma impurity content, and by inducing the formation of magnetic islands in the outer plasma flux surfaces, thereby increasing transport.

An illustration of the toroidal field coil set is shown in Fig. II.5. The set consists of 32 discrete 8 -turn coils. The total numbers of coils, the number of turns per coil, the coil radius, and the design of the coil current feeds were all set by the condition to limit the radial field error due to spatial toroidal field ripple to less than $0.3 \%$ of the poloidal magnetic field at the plasma surface. 


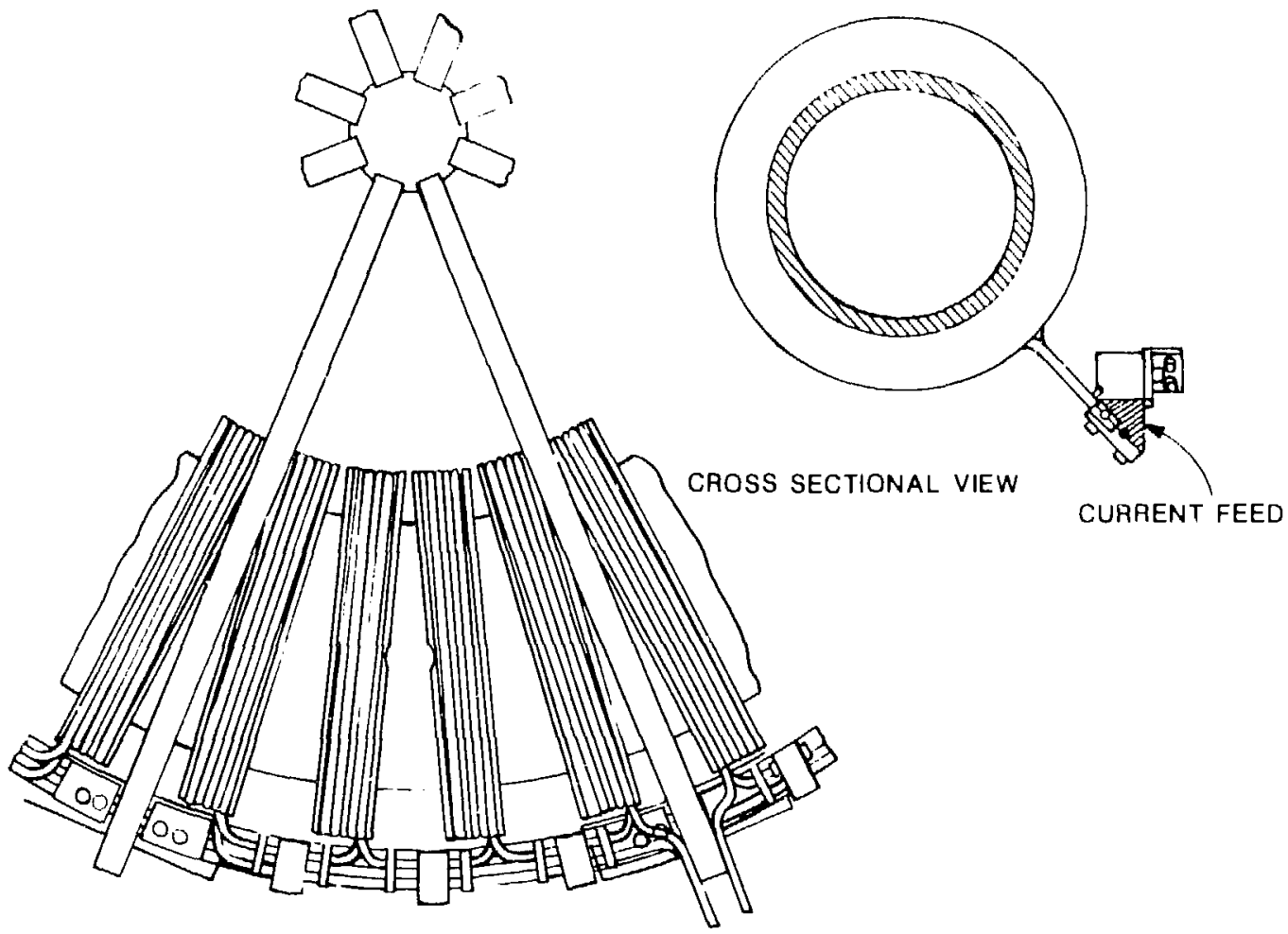

Fig. II. 5 .

ZT-P toroidal field coil set.

\section{ELECTRICAL DESIGN}

The electrical circuits for $\mathrm{ZT}-\mathrm{P}$ are shown in Figs. III.1 and III.2.5 The main energy storage system uses capacitor banks that are switched by ignitrons. The required energy to flat-top the plasma current is supplied by a power crowbar capacitor bank which is coupled to the main circuits through low-leakage-inductance, low-resistance transformers. Two equilibrium power supplies distribute the current in the equilibrium coils to provide the correct time dependent vertical field.

The poloidal field circuit is schematically shown in Fig. III.1. The main capacitor bank used for discharge initiation consists of four sections, each with twelve $170-\mu \mathrm{F}, 10-\mathrm{kV}$ capacitors connected to operate at $\pm 20-\mathrm{kV}$ with respect to ground. The power crowbar capacitor bank used for discharge

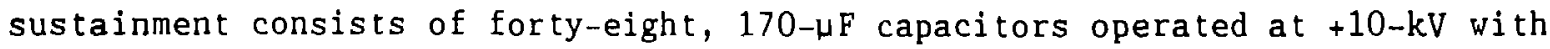
respect to ground. All capacitor banks contain small. damping resistors to 


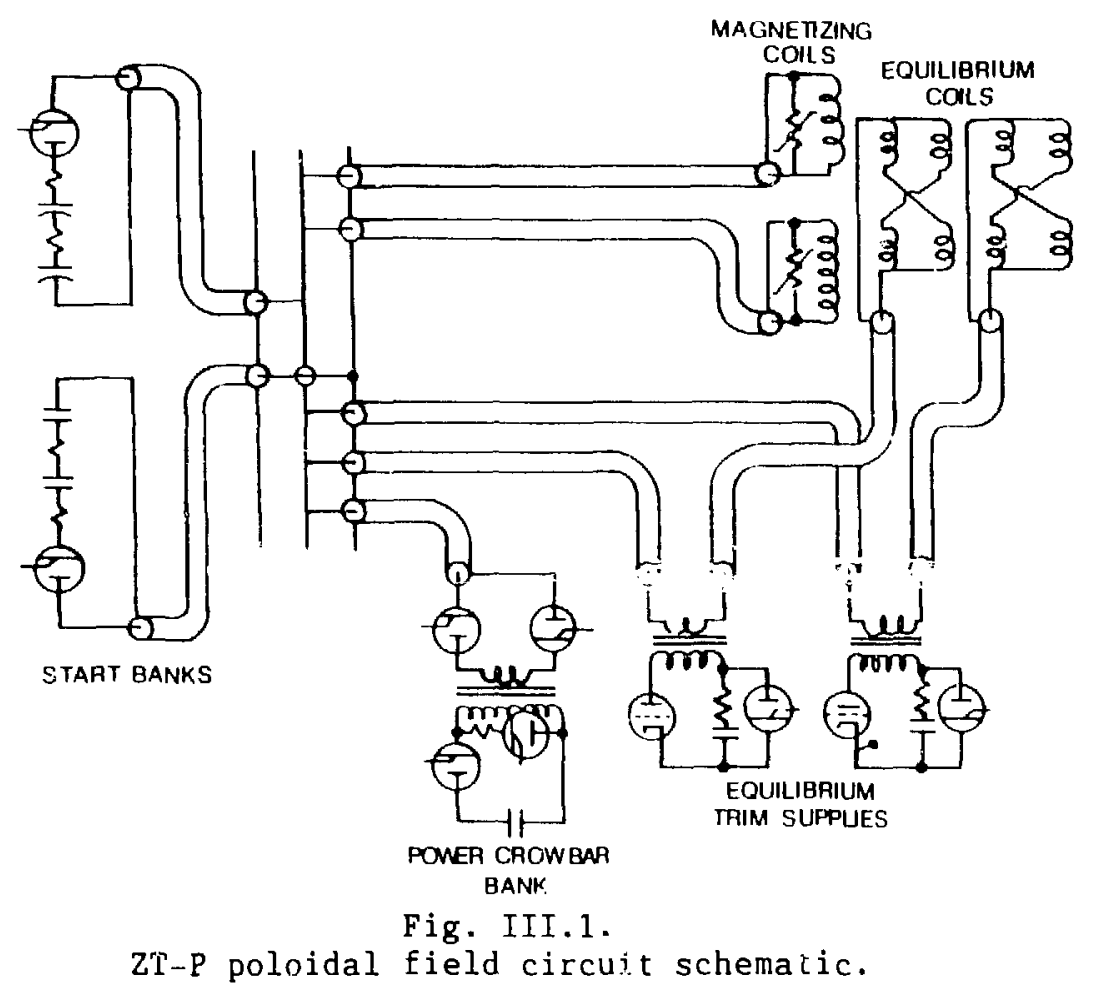

provide a damped waveform in the event of a system dump and to limit fault currents.

The power crowbar transformer, which is used to match the power crowbar bank to the primary circuit, requires a 6 volt-second flux swing and a turns ratio of $4: 1$. A transformer core with a $0.376 \mathrm{~V}-\mathrm{s}$ bipolar flux swing was selected, requiring 72 primary turns and 18 secondary turns.

Protective end-of-shot-dump (EOSD) size-D ignitrons dump the power crowbar energy in the evert of plasma termination or failure to achieve plesma breakdown. They may also be used to terminate a normal shot by ramping the current gradually to zero. The EOSD circuits have resistance values small enough to effectively shunt current from the equilibrium coils and large enough to damp oscillations.

The toroidal field circuit is shown in Fig. III.2. The main capacitor bank consists of twenty-four, $60-\mu \mathrm{F}, 10-\mathrm{kV}$ capacitors that are switched by back-to-back ignitrons. On each capacitor, 0.8-ohm resistors reduce ringing when the crowbar ignitron is closed. The power crowbar bank is a single 60- $\mu \mathrm{F}$ capacitor, coupled through an 80:1 power crowbar transformer. The transformer core has $0.128 \mathrm{~V}-\mathrm{s}$ bipolar, with 240 primary turns and 3 secondary turns. This transformer was designed to have very low secondary leakage inductance. 


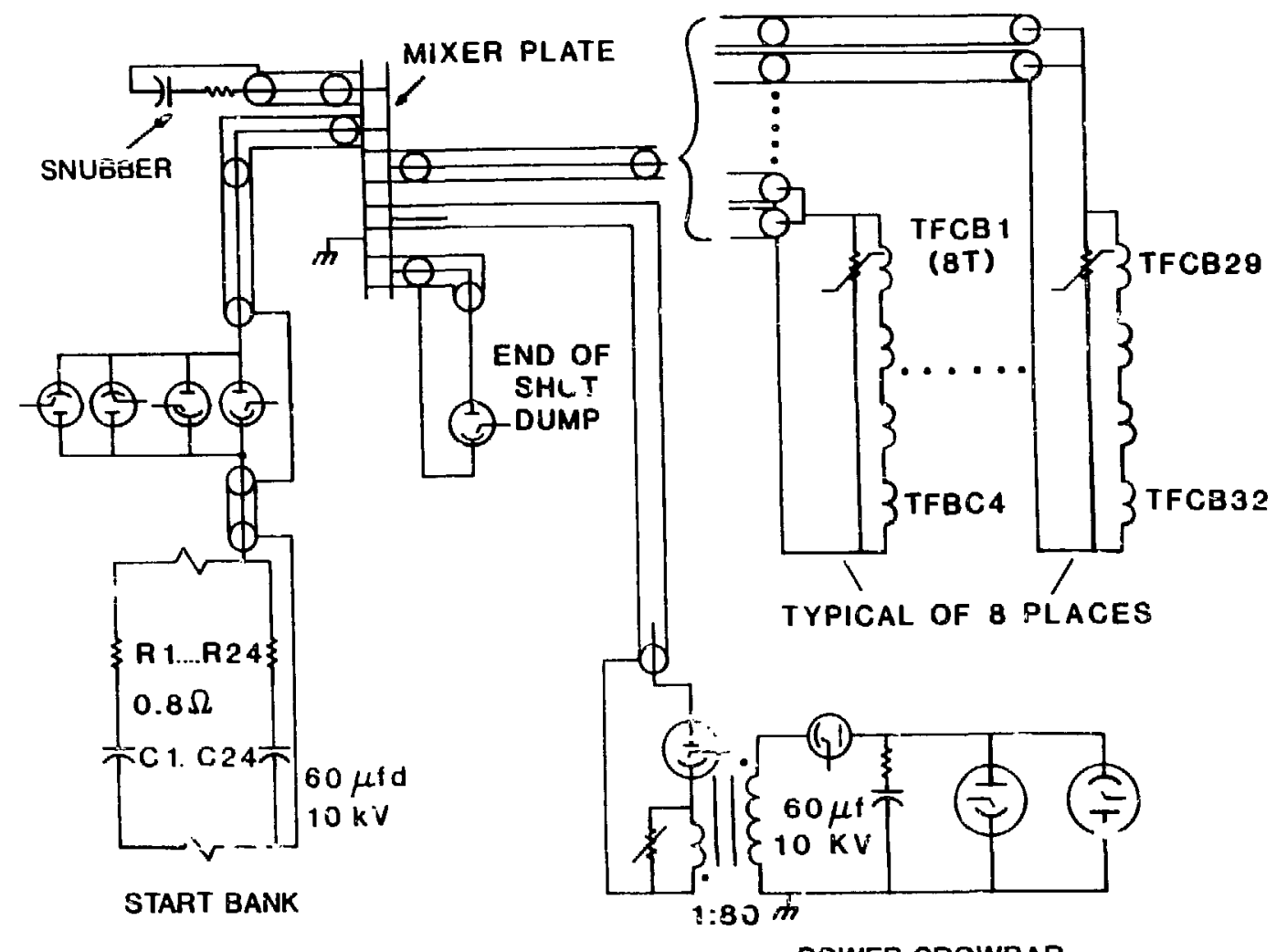

Fig. III.2.

POWER CROWBAR

ZT-P tcrordal field circuit schematic.

In both circuits, coaxial cables (17/14 HR) provide the interconnections between components. The use of coaxial cable allows considerable flexibility in locating the components and in accommodating future circuit changes.

A schematic of the equilibrium feedback system is shown in Fig. III.3. The equilibrium power surolies are transformer-coupled hard-tube amplifiers, with an output stage consisting of six parallel ML8518 magnetic beam focus triodes in a grounder cathode configuration. Each tube has a plate current of approximately $170 \mathrm{~A}$, that is supplied by a common 20-kV, 255- $\mathrm{FF}$ (51 KJ) capacitor bank. The ML8618's are driven by a fiber-optic controlled hot-deck amplifier with a single 3CX20-000A3 output tube. Solid state drive circuitry is direct-coupled to the $3 \mathrm{CX} 20-000 \mathrm{~A} 3$ grid, with fiber-optic links providing voltage isolation from grourd. 


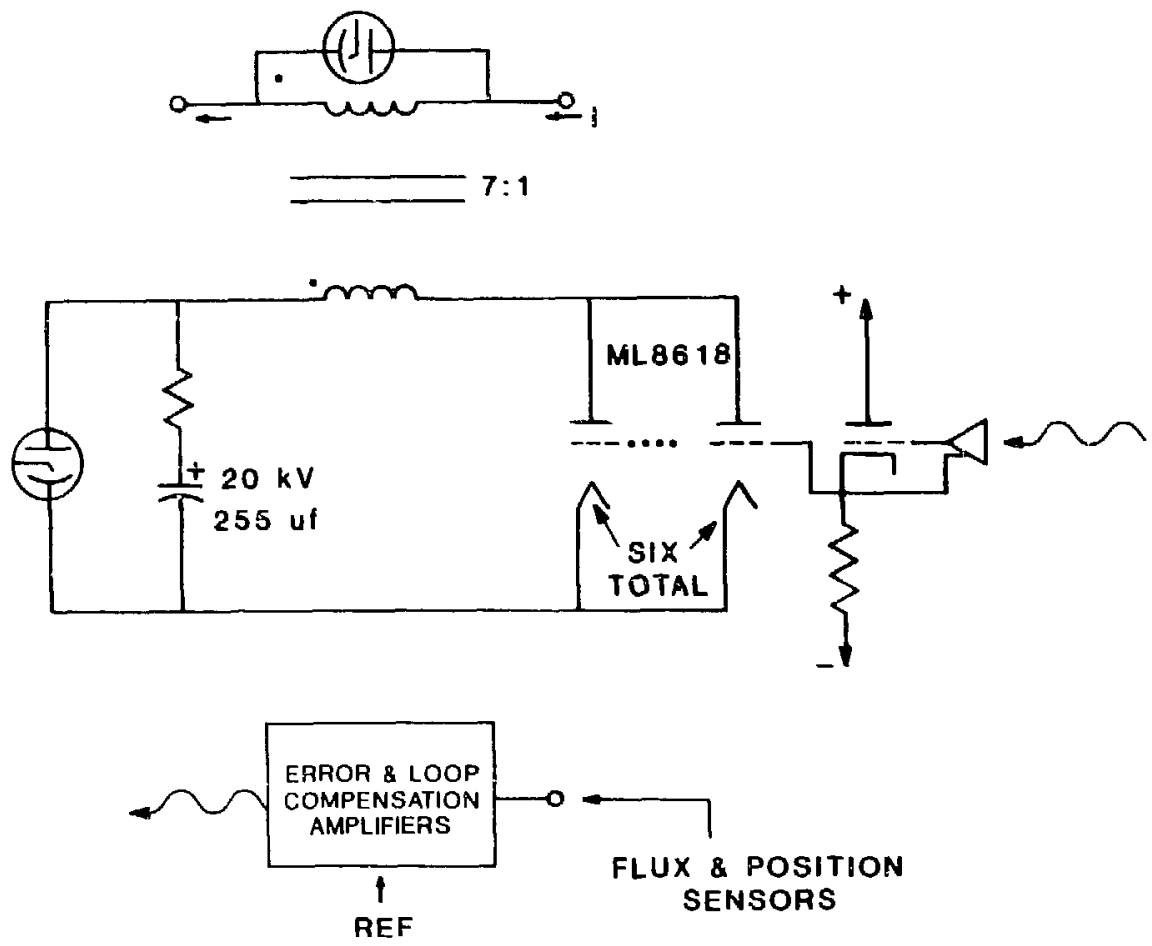

Fig. III.3.

Equilibrium feedback amplifier schematic.

IV. TORUS ASSEMBLY

An important ZT-P charter is to study the effect of boundary conditions on R'P stability. Another is to test various first wall and iimiter designs at ultra high current densities $\left(10-20 \mathrm{MA} / \mathrm{m}^{2}\right)$. In order to efficiently execute these diverse experimental goals, ZT-P was designed to easily accommodate different torus assemblies. Utilizing a modular support structure design, torus assemblies can be interchanged in a few days.

The first torus assembly, designated Phase 1, will be used during machine initiation and prototype testing. It consists of four conceptually discrete components:

1) the vacuum liner, which provides vacuum integrity for the plasma discharge;

2) the limiters, which protect the ziner from high power loading due to plasma-wall interactions; 
3) the conducting shell, which provides both equilibrium and stability to the discharge; and

4) the dielectric shell, which is the outer mechanical reierence surface for the torus assembly and provides mechanical support for the toroidal field coils.

These components are discussed in more detail below.

Vacuum Liner and Limiters

An assembly drawing of the liner is shown in Fig. IV.1. The liner consists of twelve convoluted, $0.254-\mathrm{mm}$-thick stainless steel bellows sections and four $0.450-\mathrm{mm}$-thick diagnostic sections. The thickness of the diagnostic sections was chnsen such that the poloidal resistance per unit of toroidal length was a constant, which results in a uniform poloidal current density in the liner during startup. The assembled liner, shown in Fig. IV.2, has a minor i.d. of $13.6 \mathrm{~cm}$, a minor $0 . d$. of $15.7 \mathrm{~cm}$, and a major radius of $45 \mathrm{~cm}$. The insulation wrapped arcund the liner is composed of high temperature silicone rubber and provides both electrical. insulation and mechanical support betweer the liner and the shell.

Figure IV.3 illustrates the two types of graphite ring limiters used for wall protection. Two sjde-by-side type A limiters straddle each bellows-tobellows weld for a total of 16. In addition, three side-by-side cype A limiters provide protection directly under the conducting shell gap and, for symmetry, toroidally opposite the gap at $180^{\circ}$. Type B limiters are used to protect the diagnostic section to bellows welds, with one limiter over each weld for a total of 8 . The 30 graphite limiters have a total effective area of $4800 \mathrm{~cm}^{2}$ which is approximately $20 \%$ of the total vacuum liner wall area.

The choice of poloidal ring limiters for liner protection in ZT-P was based on the results from previous experiments on ZT-40M.6,7 In these experiments, poloidal rings were located at $90^{\circ}$ toroidal intervals. The rings protruded approximately $1 \mathrm{~cm}$ into the plasma beyond the vacuum bellows and absorbed roughly 50\% of the energy conducted to the wall. Earlier experiments, ${ }^{6}$ with a single poloidal ring and a mushroom shaped diagnostic probe, indicated that approximately $80 \%$ of the energy flow to the diagnostic probe was in the direction of the electron drift. The cumulative effect of four poloidal limiters versus one poloidal limiter was evidenced by a $24 \%$ drop in the energy absorbed by the outboard segment of the ring with four rings in 


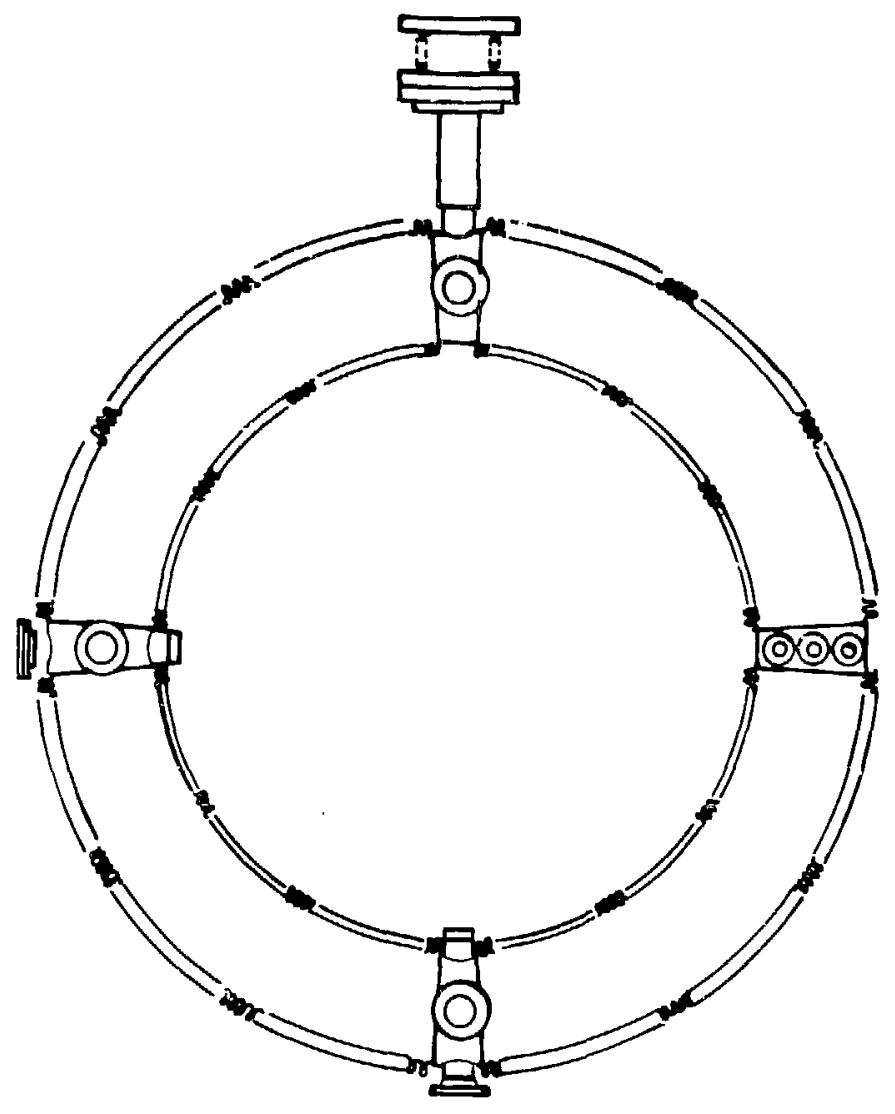

Fig. IV.1.

The ZT-P Phase 1 liner.

place. These two observations suggest the existence of a population of wellconfined electrons with a drift velocity much higher than the thermal velocity characteristic of the edge electron temperature. 7 Damage to the liner is, therefore, hypothesized to result from the interaction of these well-confined electrons with the liner.

ZT-P will operate with essentially the same magnetic field pitch at the liner and edge electron temperature as ZT-40M. These facts, coupled with the low field errors in ZT-P, should result in better confinement properties in the edge plasma than in $2 \mathrm{~T}-40 \mathrm{M}$. Thus, poloidal limiters spaced toroidally at $30^{\circ}$ intervals should provide adequate liner protection during normal oneration. Additional poloidal rings in the area of the poloidal field gap in the conducting shell also provide supplemental local protection. 


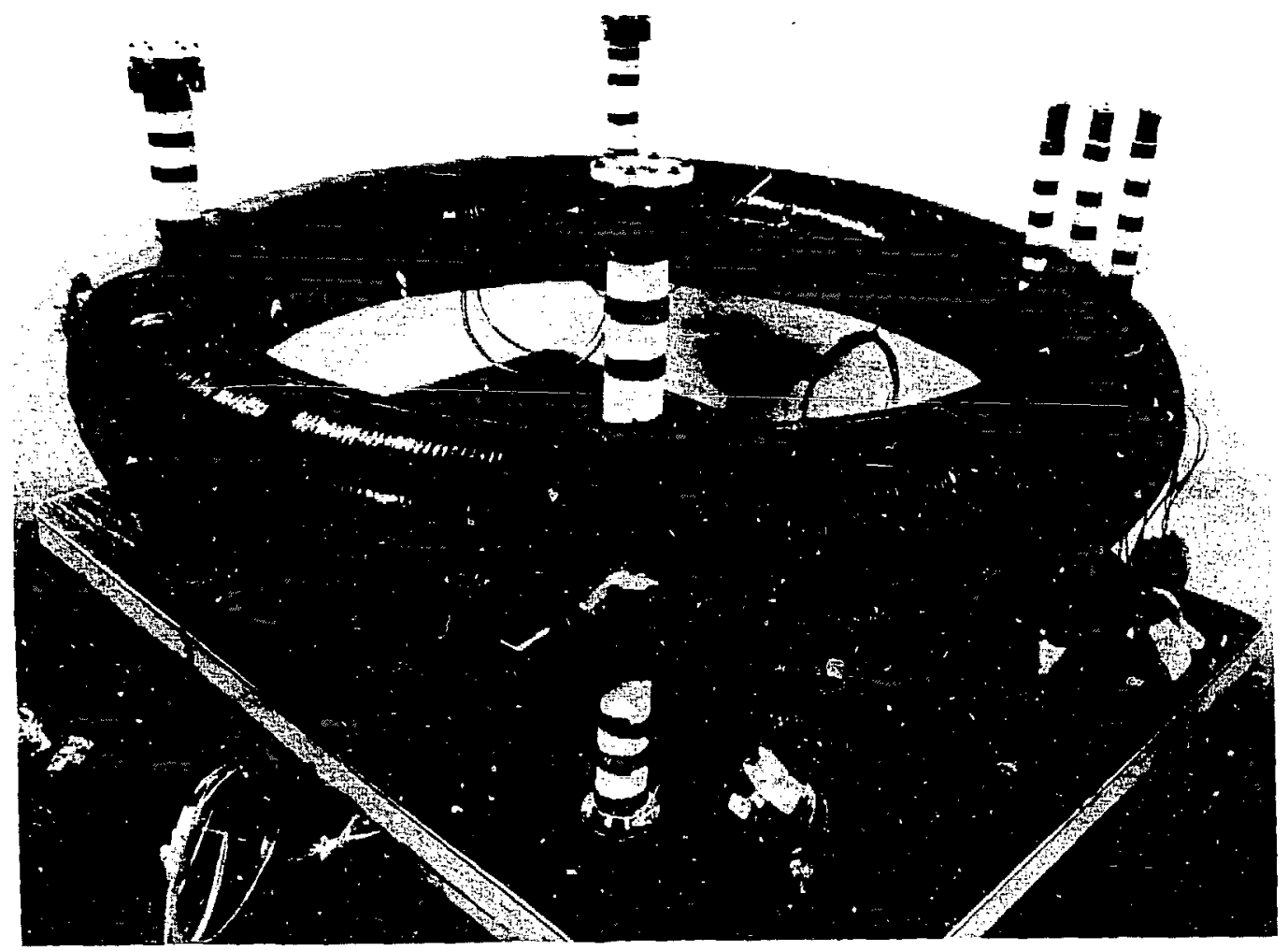

Fig. IV. 2 .

Assembled liner and insulation.

\section{Conducting Shell}

The conducting shell provides both equilibrium and stability to the RFP discharge. In Phase I of ZT-P operation, it is composed of two 1.27-cm-thick cast aluminum halves. A picture of the bottom shell casting after machining is shown in Fig. IV.4. For assembly, the shell halves are joined at the equatorial plane by machine bolts. At the outer major radial joint, a 3.2-mmthick copper gasket provides good electrical continuity between tile top and bottom halves. At the inner major radial joint, a 3.2-mm-thick polyethelyne gasket provides a gap that allows toroidal magnetic flux to enter the discharge.

In addition to the toroidal gap, the shell has a 4.7-mm-wide poloidal gap cut at one toroidal location to allow entry of poloidal magnetic flux. This gap is insulated for up to $5 \mathrm{kV}$ by a polyethelyne gasket to prevent arcing during plasma initiation, sustainment, and termination. 

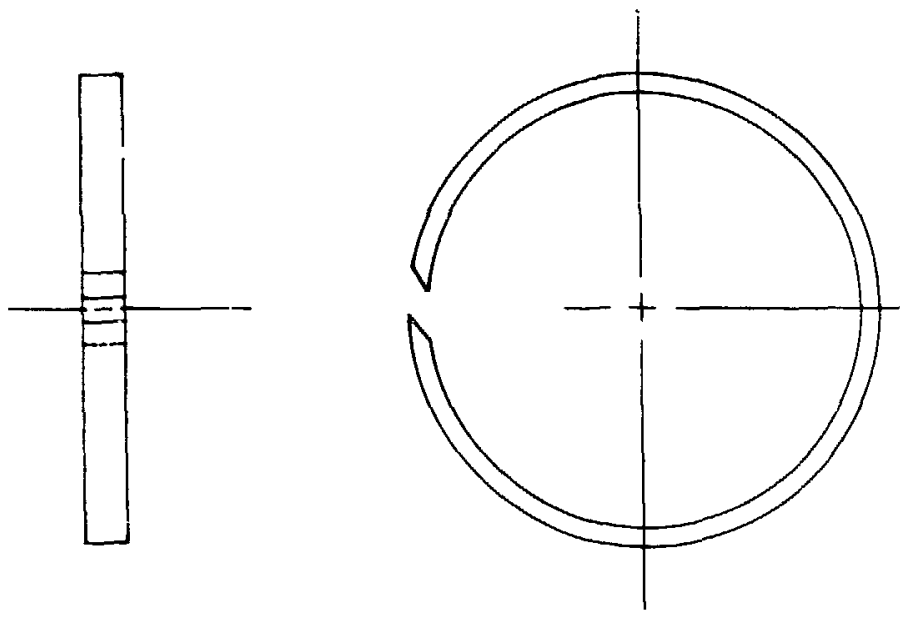

(a)
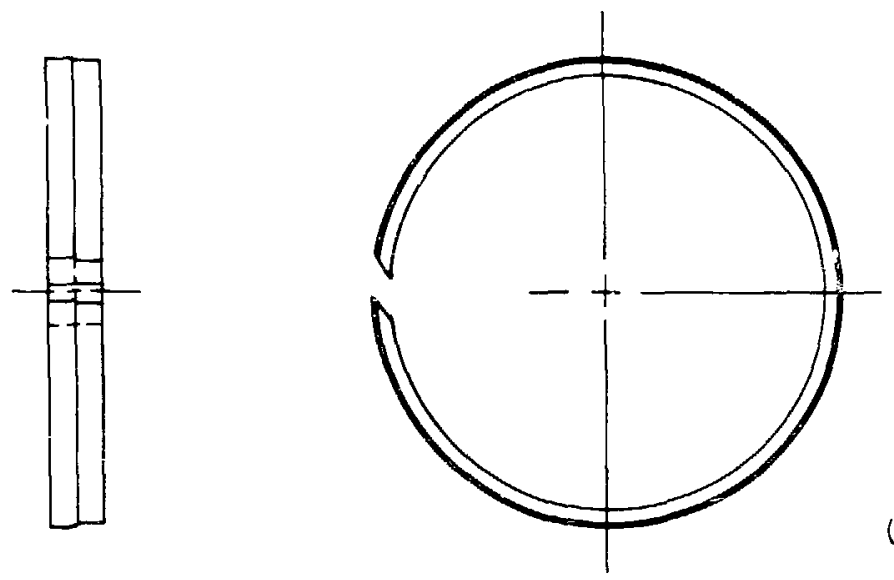

(b)

Fig. IV. 3 .

ZT-P graphite ring limiters. The top (bottom) figures illustrate limiters designated Type A (Type B), respectively. 


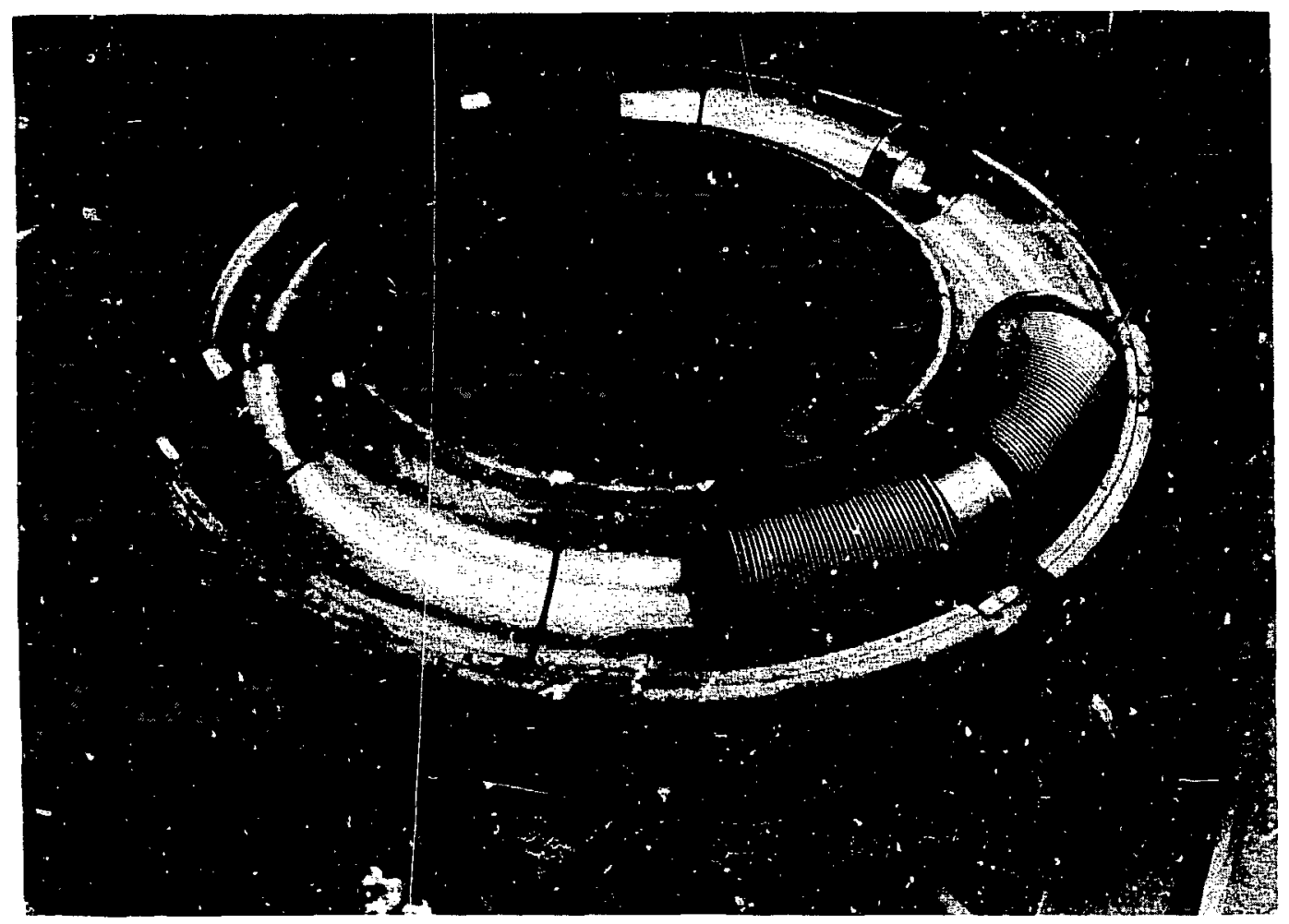

Fig. IV.4.

Phase 1 conducting shell.

\section{Dielectric Shell}

Present generation experiments indicate that RFP plasma equilibrium can probably be maintained without a conducting shell, provided active equilibrium control is utilized. However, the conducting shell's role in providing plasma stability is uncertain and subject to experimental investigation. ZT-P was designed to investigate these boundary condition studies by using a dielectric shell to produce the mechanical reference surface for the torus assembly as well as support the vacuum liner and toroidal field coils. In this way, conducting shells of various thickness can be easily incorporated in the torus assembly without affecting other torus assembly components. The dielectric shell is fabricated from a G-10 figerglass epoxy layup and then machined to the desired tolerances. The resulting shell is nominally $1.27 \mathrm{~cm}$ thick. 


\section{MACHINE STRICTURE}

The ZT-P mechanical support structure is illustrated in Fig. V.1. The structure consists of 8 radial G-10 fiberglass-epoxy bulkheads mounted on a base frame of Extren structural sections, and tied to a vertical center beam that runs along the major axis of the torus. The bulkheads are braced laterally to each other, principally by the 14 magnetizing coils and 8 equilibrium coils that comprise the poloidal field system. Adjacent to the torus, the bulkhead sections are 1 inch thick. Above and below the equilibrium coils, the bulkhead thickness is 0.75 inches. The bulkheads employ a segmented design for easy assembly and disassembly. The individual bulkhead segments are joined by bolts and, in a few cases, by bridging stiffeners.

The poloidal field coils are wound from insulated OFHC CD 104 copper conductor. Turn-to-turn insulation relies on Uni-Scotchply type 1002 over Kapton H-Film. To complete the coils, the windings were wrapped with fiberglass epoxy and cured. The 32 toroidal coils are mounted on the outer fiberglass-epoxy dielectric shell. Supported within the dielectric shell is a 0.5-inch-thick segmented T-356 cast aluminum shell which in turn holds the silicone lubber covered 321 stainless steel vacuum liner. Sector mounts on the dielectric shell ailow boiting of the torus assembly to the bulkhead at the pass-through locations.

During operation, ZT-P is subject to internal loads from coil reactions and from forces transmitted from the torus assembly to the bulklieads ar the at tach points. The latter forces result from vacuum loading of the torus plus toroidal coil effects. The interaction of these forces was analyzed by the finite element method, and a summary of that analysis is discussed below.

\section{FEM Structure/Analysis}

An extensive static linear analysis of the ZT-P support structure was made using ABAQUS, a finite element code. Figure V.2 shows the model mesh which used approximately 900 nodes and represents a $45^{\circ}$ symmetrical section of the machine. The analysis included bulkheads, coils, connecting beams and plates, and loads transmitted from the dielectric shell to the bulkheads. 


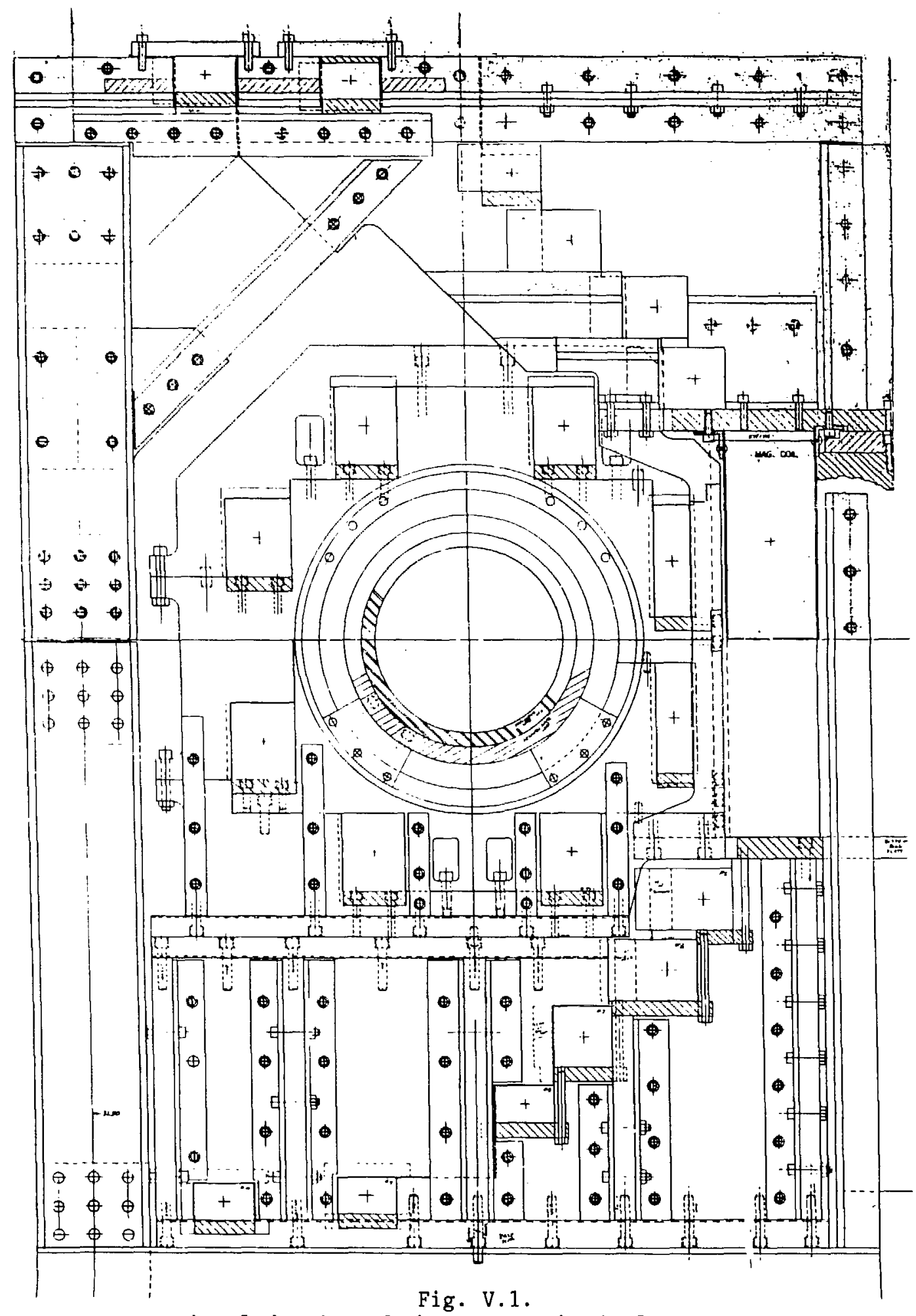

Cross-sectional drawing of the $2 \mathrm{~T}-\mathrm{P}$ mechanical support structure. 

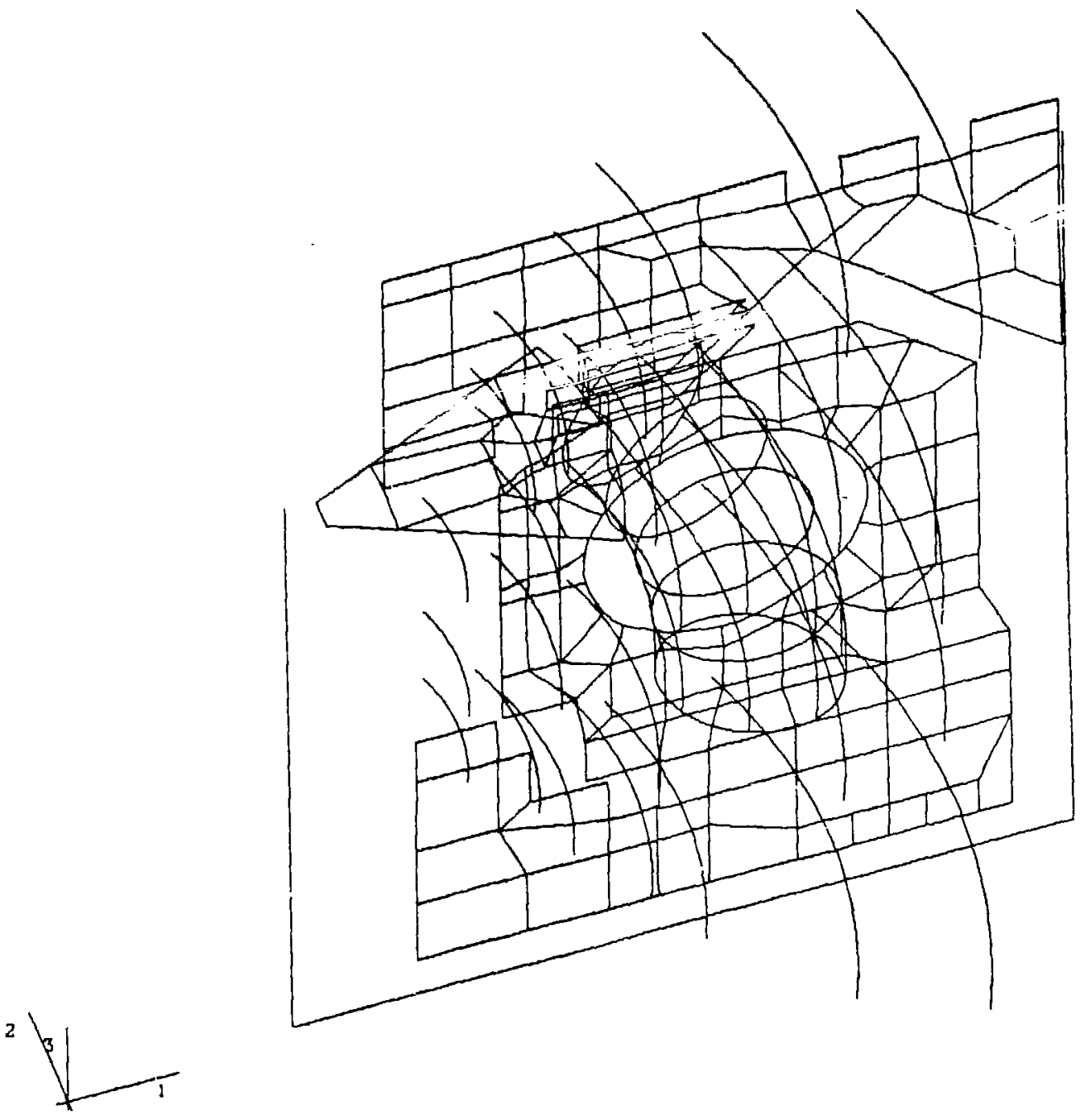

Fig. $V .2$.

Isometric view of the finite element mesh used for the support structure analysis.

Figure V.3 shows the calculated Tresca stresses for the bulkhead. No significant strength problems were uncovered in the analysis, but fairly high deflections (up to 0.051 inches) were encountered in the initial design. Figure V.4 shows, at a magnification of 20 , deflections found in the model. As a result of this analysis, the final design was modified to limit movement of the structure under load, particularly in the upper bulkhead area. 
TRESCA EQUIVALENT STRESS

$\begin{array}{cl}\text { I.D. } & \text { VALUE } \\ 1 & -2000.00 \\ 2 & -857.14 \\ 3 & 285.71 \\ 4 & 1428.57 \\ 5 & 2571.43 \\ 6 & 3714.29 \\ 7 & 4857.14 \\ 8 & 6000.00\end{array}$

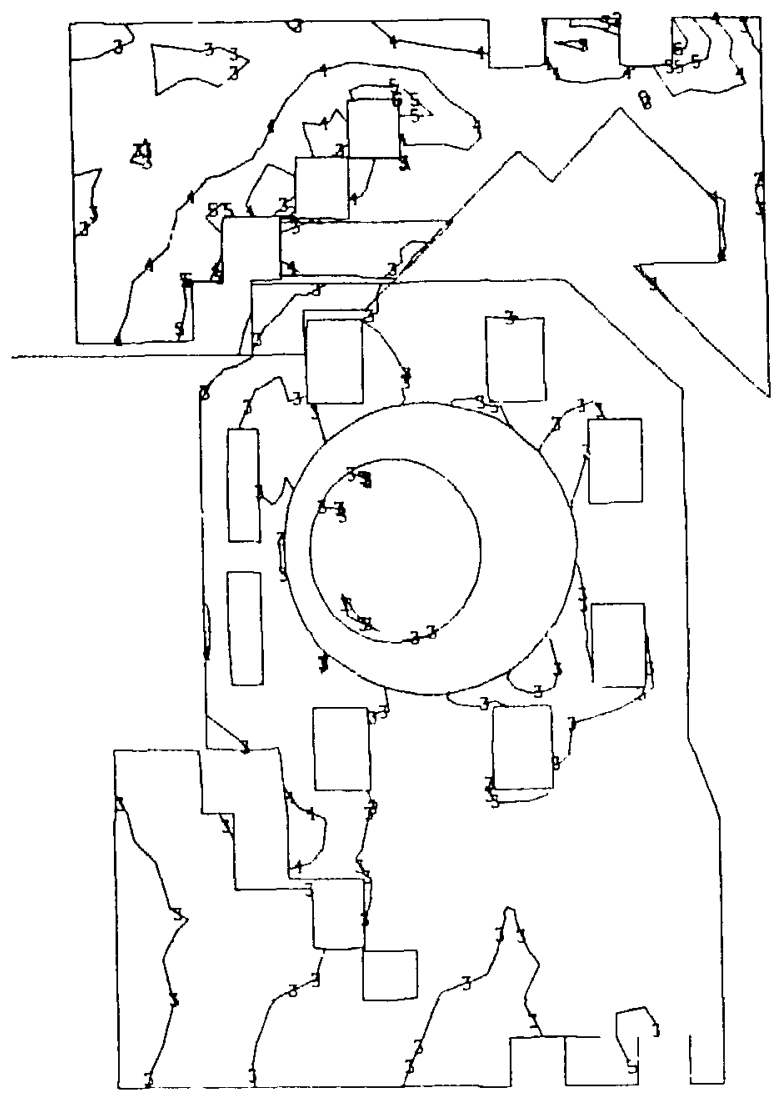

Fig. V.3.

Tresca stress plot of a bulkhead section under full load.

\section{VACUUM SYSTEM}

\section{High Vacuum System}

The ZT-P high vacuum system is illustrated in the top portion of Fig. VI.1. The high-vacuum line consists of a Turbomolecular Pump (TMP) pumping stack interfaced to the torus through a 14.8-cm-i.d., 134-cm-long duct; and a 8.3-cm-i.d., 10.2-cm-long bellows assembly. The vacuum liner is connected to the bellows through a special pumping port. The bellows section mechanically decouples the pumping system from the liner and accommodates the dimensional changes caused by heating the liner and/or the high-vacuum system. 


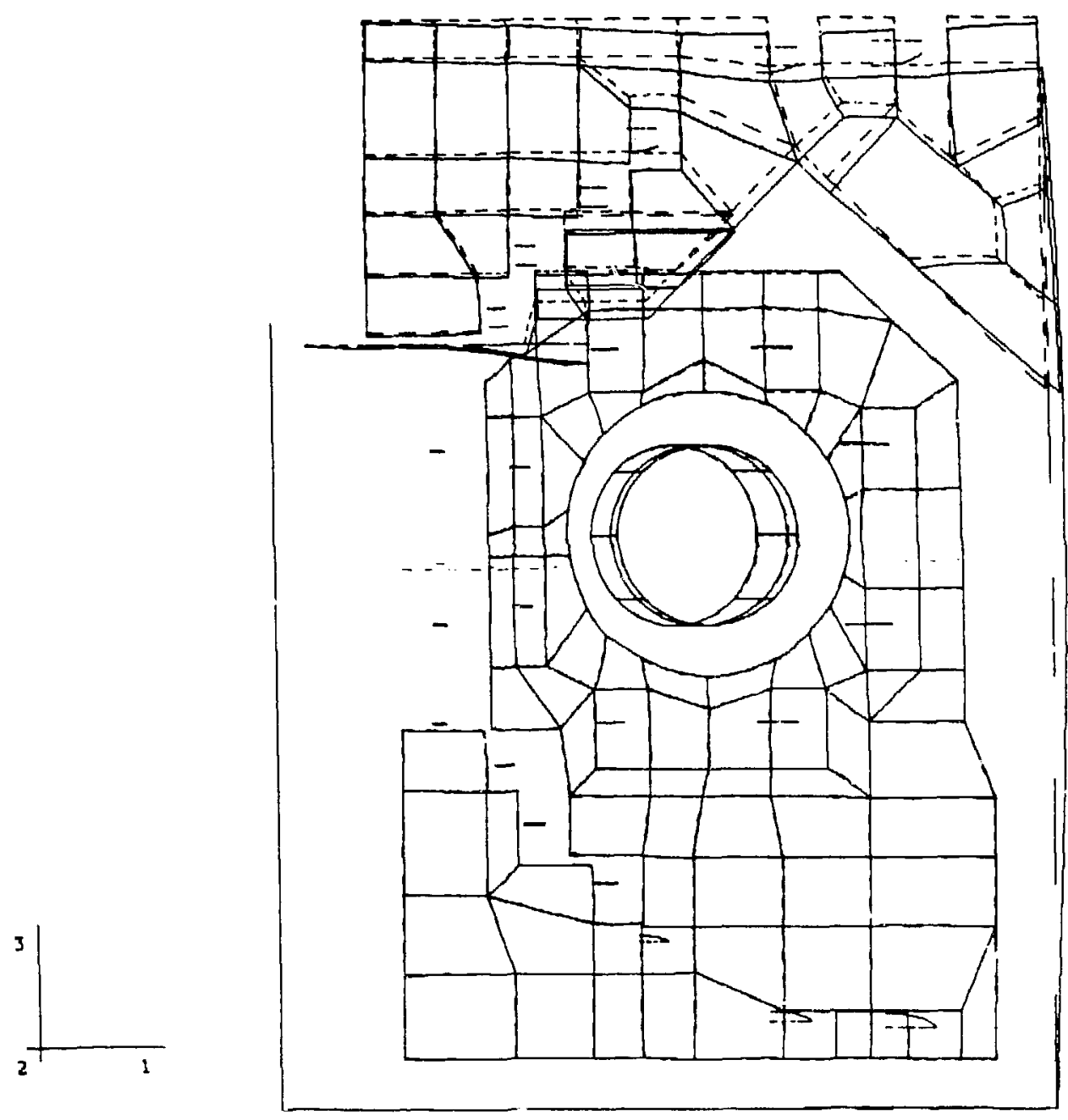

Fig. V.4.

Resulting deformation of structural mesh under full load (20X magnification). The dashed lines mark the original mesh while the solid lines show the displaced mesin.

In order to minimize field errors resulting from a hole in the conducting shell, ZT-P uses a single pump port. This port consists of two sectiuns of stainless steel tubing. Nearest the liner, the port has a $4.0-\mathrm{cm}-\mathrm{i} . \mathrm{d}$. and is 3.5 -cm-long. Joining this tube to the bellows assembly is a $14.6-\mathrm{cm}-$ long section with an i.d. of $5.1 \mathrm{~cm}$. This port/stack configuration results in a conductance of $\sim 30 \mathrm{l} / \mathrm{s}\left(\mathrm{N}_{2}\right.$ at $\left.20^{\circ} \mathrm{C}\right)$ and is the limiting conductance in the system. The total pumping speed $\left(\mathrm{S}_{\mathrm{T}}\right)$, characteristic pumping time $(\tau)$, and the estimated base pressure $\left(P_{U}\right)$ for $\mathrm{N}_{2}$ at $20^{\circ} \mathrm{C}$ with a TMP pumping speed of $500 \mathrm{l} / \mathrm{s}$ are as follows: $\mathrm{S}_{\mathrm{T}} \approx 251 / \mathrm{s}, \tau \approx 2 \mathrm{~s}$, and $\mathrm{P}_{\mathrm{U}} \approx 1.4 \times 10^{-8} \mathrm{mbar}$. 


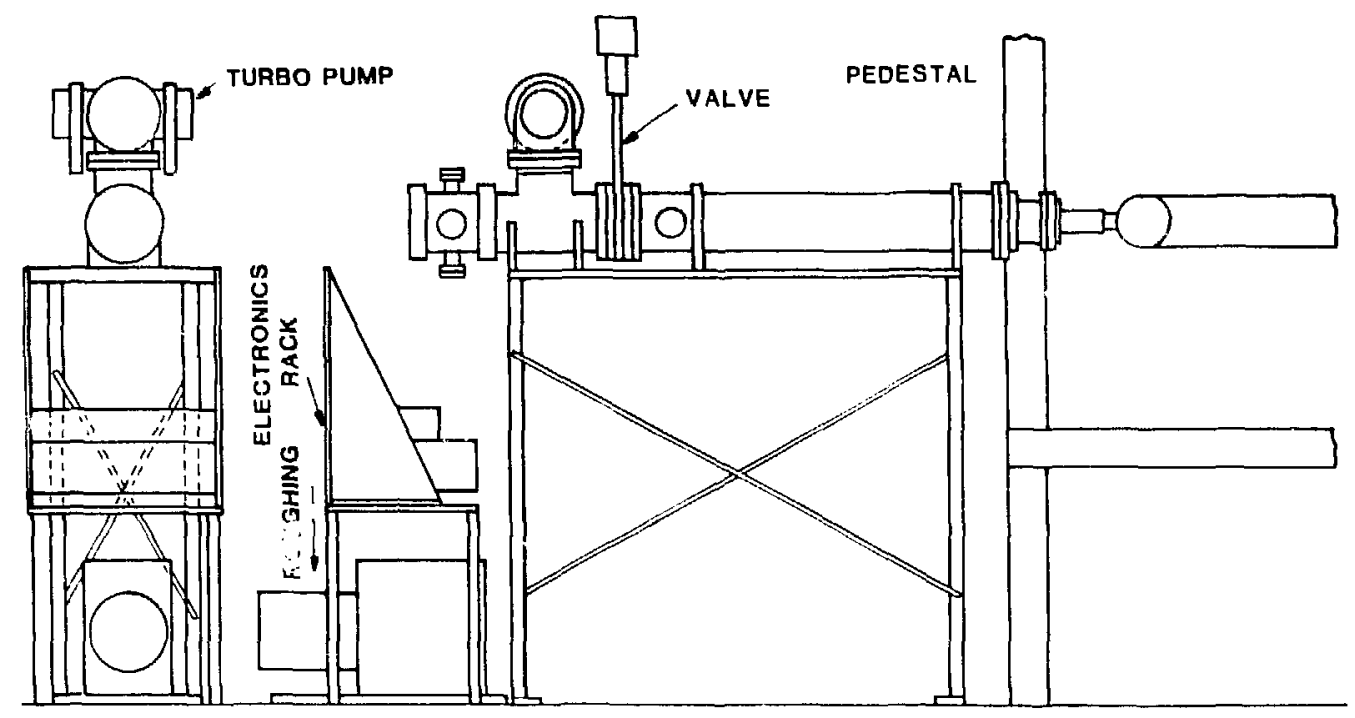

Fig. VI. 1 .

The $\mathrm{ZT}-\mathrm{P}$ vacuum system.

The vacuum liner and pumping station are pumped from atmospheric pressure with the TMP/roughing pump combination. At the start of pumpdown, the TMP and roughing pump are turned on simultaneously. The TMP rotational speed is always sufficient to prevent the backstreaming of oil from the roughing pump. The vacuum liner and pumping station are also designed tc be bakeablr to at least $150^{\circ} \mathrm{C}$. Metal-sealed joints are used throughout the high-vacuum pumping system, and 0-rings are used to seal the vacuum liner ports and the gate seals on the high vacuum valves.

The vacuum liner has an estimaied volume of 49.61 and an area of $4.0 \times 10^{4} \mathrm{~cm}^{2}$. Assuming an outgassing rate of $5.3 \times 10^{-12} \mathrm{mbar}-1 / \mathrm{s}-\mathrm{cm}^{2}$, which is appropriate for stainless steel or Inconel 625 after bakeout at $150^{\circ} \mathrm{C}$ for 24 hours, the load on the pumping system is approximately $2.1 \times 10^{-7} \mathrm{mbar}-1 / \mathrm{s}$. An additional load to the vacuum system is presented by the $4.8 \times 10^{3} \mathrm{~cm}^{2}$ of graphite limiter area in the liner. These limiters will operate at an estimated temperature of $200^{\circ} \mathrm{C}$, with a corresponding outgassing rate cf approximately $5 \times 10^{-11} \mathrm{mbar}-1 / \mathrm{s}-\mathrm{cm}^{2}$. These two major componen on the pumping system of $\sim 4.5 \times 10^{-7} \mathrm{mbar}-1 / \mathrm{s}$.

A continuous flow of hydrogen or deuterium will determine the pressure in the liner during a cischarge. Continuous operation at gas pressures of $0.1-2.0 \times 10^{-2}$ mbar will be required for RFP discharges, pulse discharge cleaning 
(PDC), and glow discharge cleaning (GDC). Provision for operation at higher gas pressures ( $\leq 10^{-1}$ mbar) may be necessary to optimize the GDC procedure. This high gas thr oughput precludes the use of any type of high-vacuum pump other than a TMP. However, the large magnetic fields near ZT-P during operation require that the TMP be located at leas $1.5 \mathrm{~m}$ from the center of the machine. This location will ensure that the magnetic field at the pump location is less than 200 gauss for under five seconds. A duty factor of 0.090 is within acceptable limits for the TMP in this magnetic field. An overall schematic of the vacuum system is shown in Fig. VI.2.

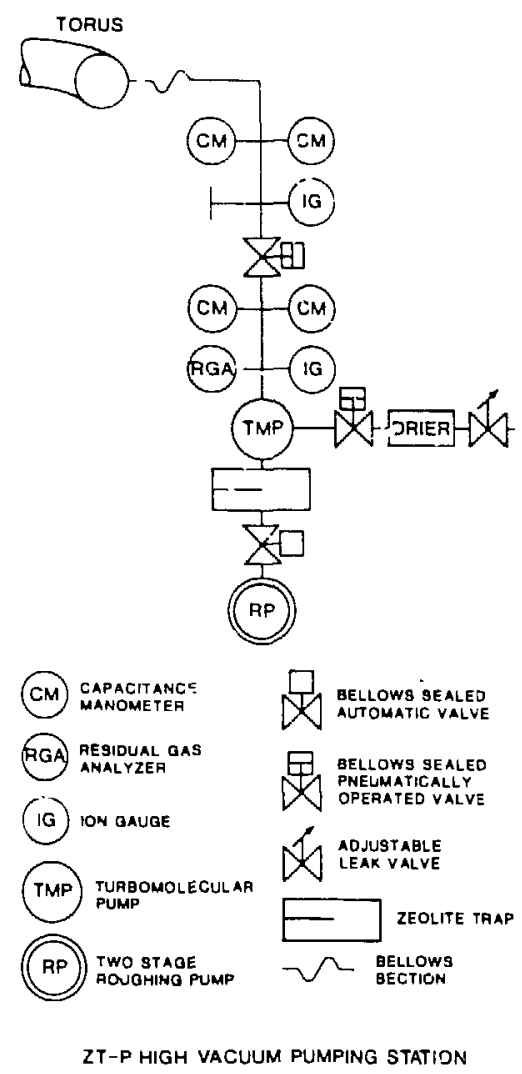

Fig. VI.2.

ZT-P vacuum system schematic. 
Gas Fill System

The gas fill system is capable of supplying high purity (99.9\%) hydrogen or deuterium for the gas injection system and for discharge cleaning procedures. Provision is also made for supplying dry impurity gases for injection. The initial fill pressure in the torus, prior to discharges, is adjustable over the range of $0.1-2.0 \times 10^{-2}$ mbar and reproducible to $\pm 2 \%$. During the discharge, gas injection rates of 100 torr-l/s are available with the provision for programming the injection pulse shape.

The gas fill system has its own roughing system, and the valves are fully interlocked to protect the torus during a malfunction. A schematic drawing of the gas fill system is shown in Fig. VI.3. Palladium purifiers are used to insure the purity of the $\mathrm{H}_{2}$ and $\mathrm{D}_{2}$ gases, and a drying system is supplied for the impurity gas. The content of the gas systems is monitored by the torus residual gas analyzer ( $R G A)$.

The gas injection system consists of two gas supply lines. During normal operation, one of the lines is used to supply a continuous flow of gas and the other is used to supply gas to an injection valve (PV-10). To achieve the high flow rates necessary for gas injection during the discharge, the injection line operates at 10-100 psi. Each gas supply line has its pressure regulated automatically by a feedback-controlled servo valve, and each supply line is electrically isolated by a high-voltage insulating break.

\section{Control System}

The design of the high vacuum system, roughing system, and gas fill system employs a fail-to-safe control philosophy. An LSI-11 subsystem control computer is used as interlock protection to prevent improper operation and to sequence events associated with the discharge and discharge cleaning procedures. Digital signals from limit switches on valves, pressure sensors, temperature sensors, power sensors, and the RGA are utilized in the interlock sequences. Both the analog and digital signals are transmitted to the controller over fiber optic links to provide electrical isolation. 


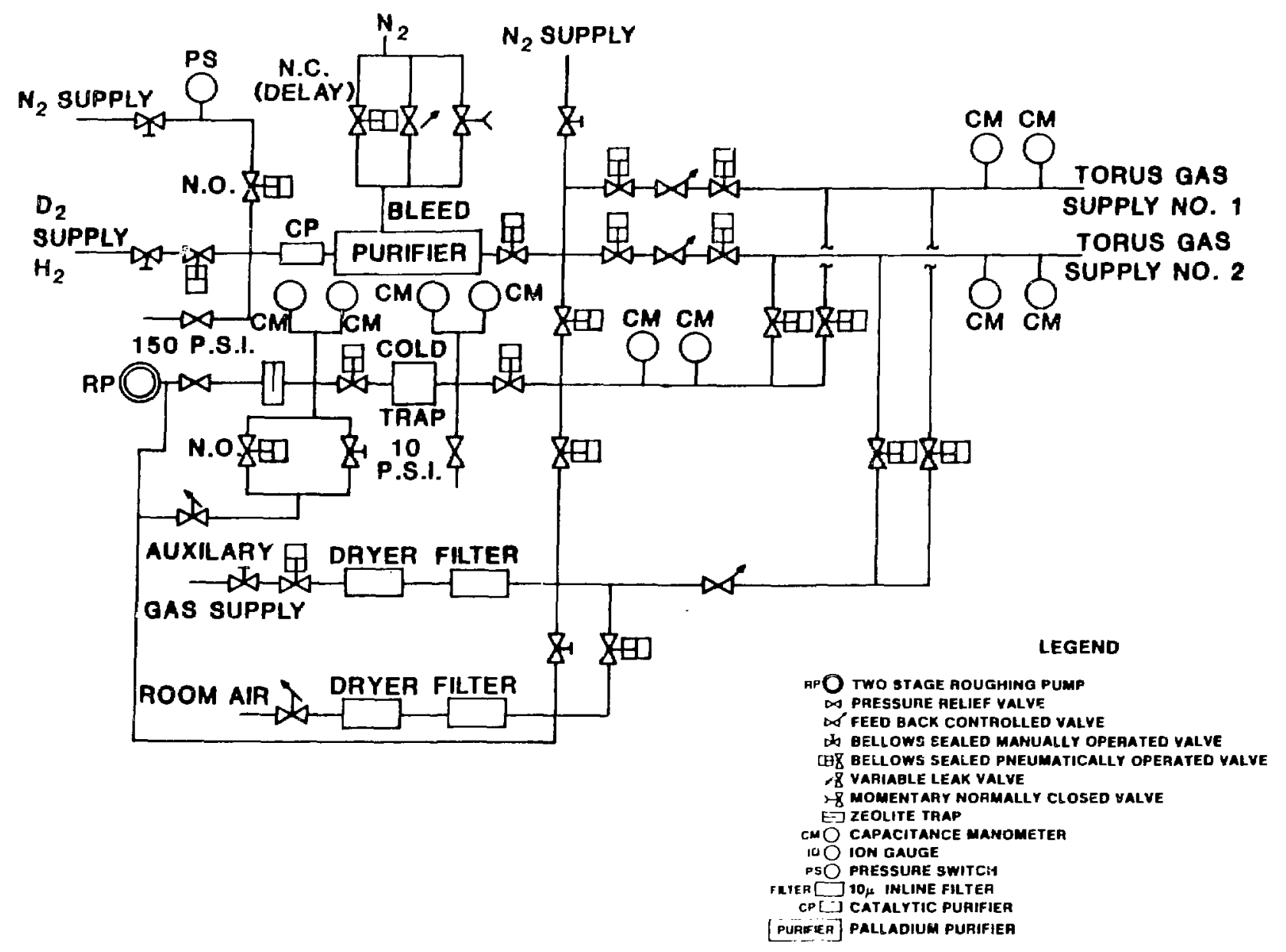

FIg. VI, 3,

Gas fill system schematic. 


\section{DIAGNOSTICS}

\section{Introduction}

The physics diagnostics on $3 T-P$ provide measurements of discharge conditions, basic plasma parameters, and properties of particular interest to the ZT-P program, nameiy plasma equilibrium and stability. The discharge conditions are measured with electrical diagnostics; that is, Rogowski coils for the toroidal current, partial Rogowski coils for the toroidal fieid, and loops for the toroidal voltage and toroidal flux. The equilibrium is determined by means of radial flux loops at the poloidal gap, and by comparing signals obtained from the toroidal voltage loops. Magnetohydrodynamic (MHD) modes are detected by means of pickup coils arranged around the minor circumference of the liner at several toroidal locations. The locations of these electrical diagnostius are shown in Fig. VII.1(a). Other electrical diagnostics are sensors to measure the current in the poloidal field windings, and loops to measure the radial flux escaping at the shell discontinuities. In addition to the electrical diagnostics, small thermocouples are used to determine equilibrium as well as plasma properties. Several sets of thermocouples are provided on the ZT-P liner as shown in Fig. VII.1(c).

The diagnostics used to determine plasma properties are a 3-chord interferometer to measure the plasma density, single-point Thomson scattering to measure the central electron temperature, and surface barrier diodes, visible and VWV spectroscopy, and a calorimeter to measure plasma radiation over a wide spectral range. The locations of these diagnostics are shown in Fig. VII.1(b). Portable diagnostics include a filter monochromator to measure line and continuum radiation in the visible, and a hard $x$-ray diagnostic for biological safety. The diagnostics enumerated in this introduction are discussed in more detail below.

\section{Electrical Diagnostics}

Most of the electrical diagnostics used for physics measurements are installed in the interspace between the shell and the liner. The distance between the outer radius of the liner and the inner radius of the shell is only $5 \mathrm{~mm}$. Since part of that space is taken by electrical insulation, the sensors must be thinner than $3 \mathrm{~mm}$. Insulation between the liner, shell and 


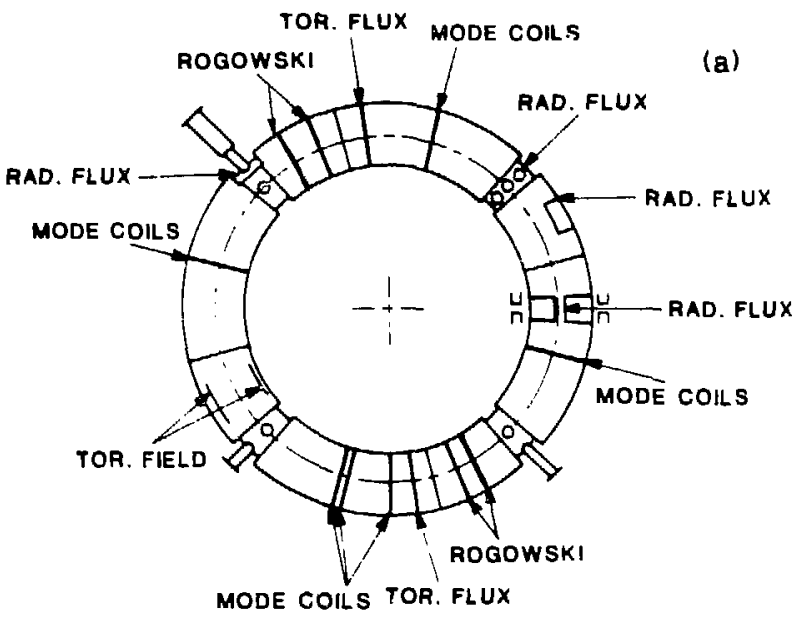

ELECTRICAL DIAGNOSTICS
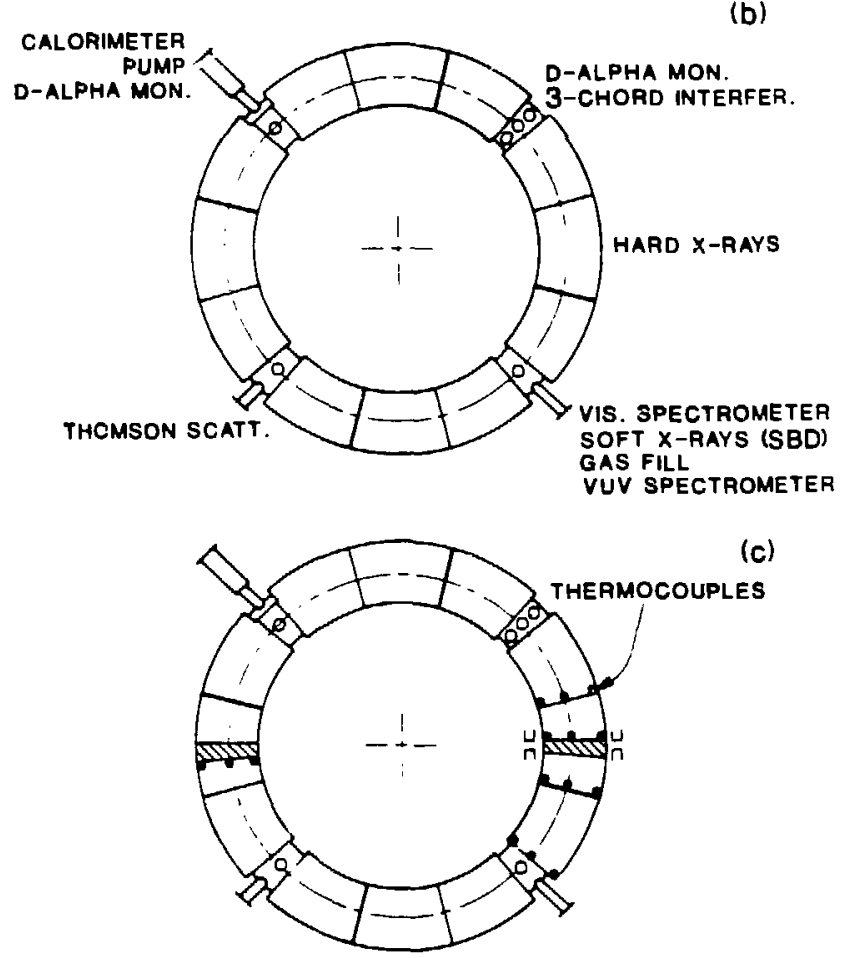

ZT-P diagnostics layout: Fig. VII.1. diagnostics, (c) Thermocouples. (a) Electrical diagnostics, (b) Optical 
diagnostics is rated at $5 \mathrm{kV}$, and all sensors are designed to withstand a baking temperature of $150^{\circ} \mathrm{C}$.

As shown in Figs. VII.1 and VII.2, some of the electrical sensors are attached directly to tre iner. Two toroidal flux loops are mounted at the bottom of liner corrugations and are positioned 180 degrees apart toroidally. The diameter of these loops is $139.3 \mathrm{~mm}$. There are 4 toroidal voltage loops at poloidal locations $0,90,180$, and 270 degrees. * Loop pairs are connected to signal conditioners, which produce average and time-integrated difference signals. These signals are used to determine the loop voltage and the global equilibrium position of the discharge.

The other interspace diagnostics are attached to the shell as shown in Fig. VII.2. There are three Rogowski coils used to measure the sum of the liner and plasma toroidal currents. Each Rogowski coil is made up of two half coils, which are taped against the inner minor circumference of the upper and lower half shells. The signals from the two half coils are combined in a resistive network at the signal conditioner. Two Rogowski coils are constructed using a printed-circuit technique, as described below. The third coil uses a conventional winding technique.

There are two so-called partial Rogowski coils, identical to the printed circuit half-coils, that are used to measure the local toroidal field. These sensors are installed near the inner and outer midplane of the torus, at the same toroidal location. These coils provide a measurement of the toroidal field in the interspace, averaged over a toroidal length of $260 \mathrm{~mm}$.

Five sets of mode coils measure the toroidal component of the magnetic field at toroidal locations 59, 149, 239, 329 and 224 degrees. There is also a set of coils measuring the poloidal component of e field at 239 degrees. Mode coils are pickup coils positioned around the minor circumference, to measure spatial Fourier components of the toroidal or poloidal field at the wall. Each mode coil set consists of four pickup coils located poloidally at 45, 135, 225, and 315 degrees. The mode coil signals are combined in resistive networks to produce sine and cosine components of possible $m=1$ MHD modes in the plasma. By changing the network resistances, one can also

\footnotetext{
"The following convention is used when referring to a location on the torus. The toroidal angle is measured from the pump port, moving clockwise around the machine as viewed from the top. The poloidal angle is measured from the outer midplane of the torus, going upward.
} 

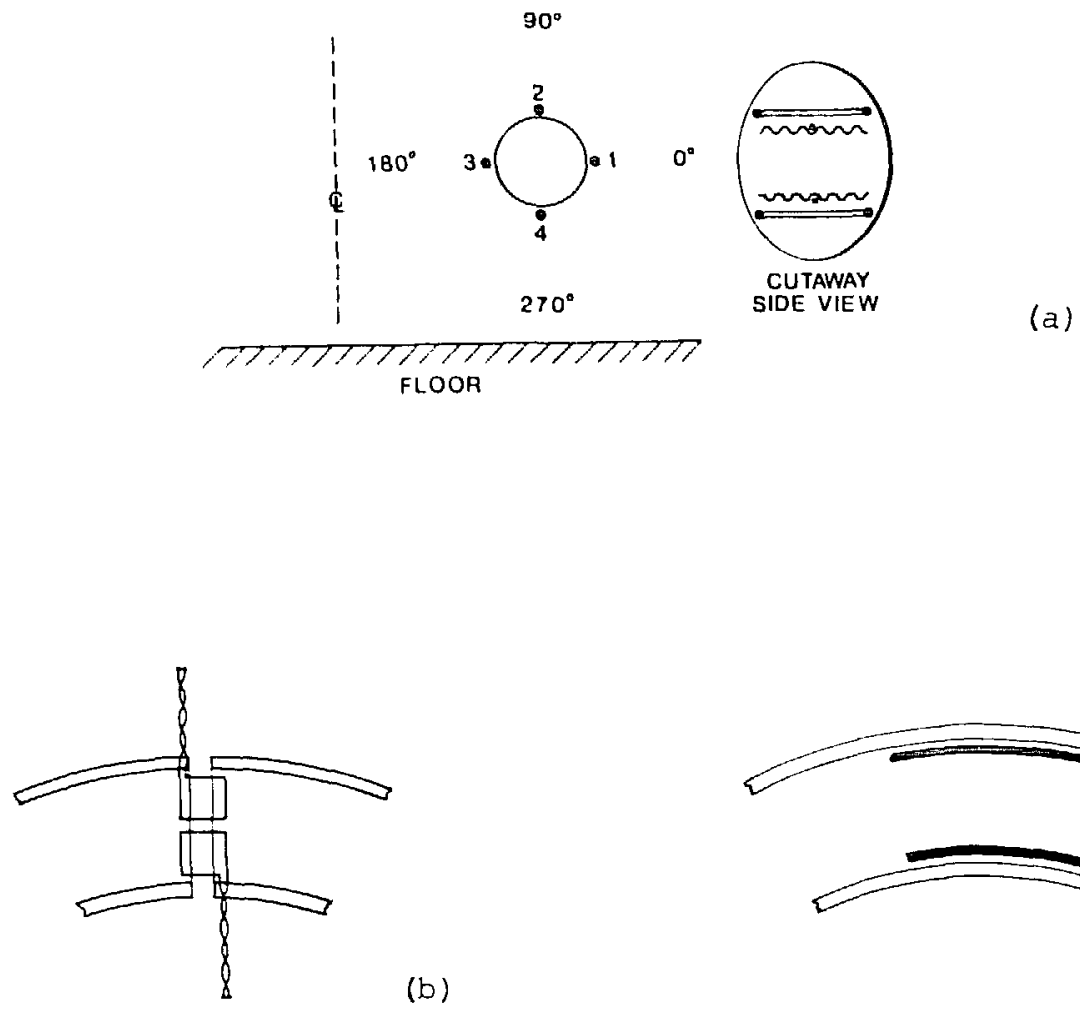

(b)

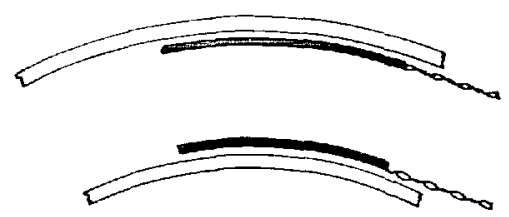

(c)
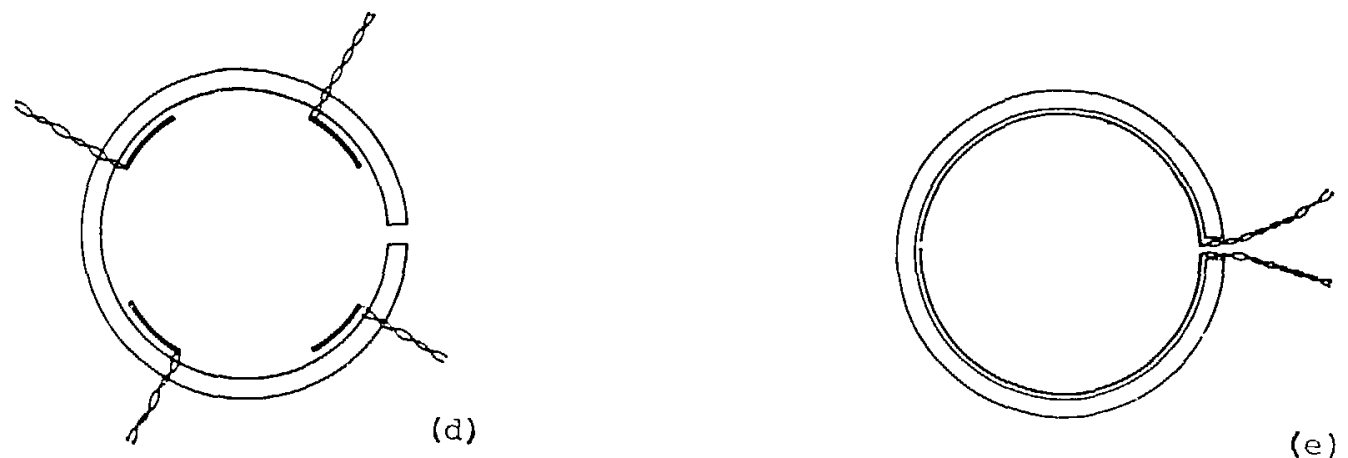

Fig. VII.2.

ZT-P interspace diagnostics: a) Toroidal voltage loops at $0,90,180$ and 270 degrees; b) Radial flux loops at the shell poloidal gap; c) Partial Rogowski coils; d) Mode coils; e) Rogowski coil. 
measure $m=0$ modes. Toroidal mode numbers of up to twelve can be observed with this system.

There are several loops designed to measure the radial flux escaping from the shell. Four rectangular loops are installed at the poloidal gap in the shell. They cover four quadrants of the minor circumference, with the first loop extending from the midplane to the top of the torus, and so on. Signals from diametrically opposite loops are combined before going to a signal conditioner. Outputs from the two signal conditioners are used to determine the plasma displacement at the gap. Other radial flux loops are located at the toroidal gap and at the pump and diagnostic ports, to sample the flux escaping from these discontinuities in the shell. External to the machine, six Pearson-type current sensors measure the currents in the poloidal field coils. This measurement is useful for plasma equilibrium calculations.

Printed-circuit coils were also selected for the mode coils. Printed circuits are, in principle, reproducible, and easy to manufacture. These coils, however, are subject to considerable bending to match the compound curvature of the ZT-P shell. This stress has resulted in discontinuities in the electrical conductance of plated-through holes used to connect one face of the windings to the other. The problem has been remedied by bridging the plated holes with soldered wires.

The Rogowski and mode coils are electrostatically shielded. The shielding is connected to a center tap on the coils. There is no shielding or center tap on the flux loops. All electrical diagnostics use shielded twisted pairs for their connections to the signal conditioners. The shielding is connected to the center tap of the sensor, if a tap is available.

\section{Liner Thermocouples}

Information about the discharge-induced local liner temperature is a useful guide for determining the discharje currents and pulse lengths that avoid significant liner damage. Such a diagnostic is important on ZT-F because of the anticipated high current densities, and because very limited access for internal plasma diagnostics is planned for future vacuum liners, in order to avoid magnetic field errors due to diagnostic holes in the liner and shell. Poloidal arrays of four thermocouples (top, outside, bottom, inside) are attached from the outside to the bellows valleys of the liner at five toroidal locations. The arrays are located in convolutions just upstream 
(relative to the electron flow direction at the wall) of the graphite ring limiters that protect the liner at the welds and at the poloidal gap.

The technique used for installation was as follows:

1. Since the liner is $0.25 \mathrm{~mm}$ thick, very fine thermocouple wire $(\sim 0.075-\mathrm{mm}$ diameter) was used to minimize thermal loading of the wall at the location of the thermocouple.

2. Chromel-Constantan junctions were used to eliminate magnetic material and provide high sensitivity $\left(64.4 \mu \mathrm{V} /{ }^{\circ} \mathrm{C}\right)$.

3. The junctions were formed using a miniature spot-welder.

4. The junctions were all calibrated in an oven and found to check well with the specified sensitivity. The resistance of the junctions was checked and found to be negligible.

5. Since the convolutions are closely spaced on ZT-P, it was necessary to apply the soldering heat from the inside of the bellows (before the bellows were welded together) using a clean soldering iron (the tip was covered with clean copper foil).

6. Soft silver solder (tin-silver, melting point $240^{\circ} \mathrm{C}$ ) and acid flux were used to appiy the junction to the convolution valley.

7. By applying the minimum amount of flux and solder for tinning to the convolution and to the junction before joining them, it was usually unnecessary to remove excess solder later. Small tweezers and dental tools were used to apply the solder, the flux, and to manipulate the junction in the convolution valley before the joining. The depth of solder was judged by observing how much of the thermocouple junction, pressed against the bottom of the convolution, was visible above the level of the solder. Generally, it was not necessary to apply additional solder and flux for the final joining following the thorough cleaning of the tinned parts with water and freon.

8. About a 2 -meter length of twisted pair of the fine thermocouple wire was used to bring thermocouple signals out to a lower magnetic field environment, where transition to heavier extension wire (also twisted) was made. 
A typical thermocouple signal is shown in Fig. VII.3. (A X200 gain differential input, light-link amplifier, described in Sec. VIII, and a LeCroy 8212A 32-channel data-logger are used for signal-processing.) The thermocouple reaches its equilibrium temperature about $75 \mathrm{~ms}$ after the shot, so that discharge-induced pickup on the thermocouple leacis is not a problem. The total peak-to-peak noise on the trace is about $0.5^{\circ} \mathrm{C}$. By averaging the data over a 50-100 ms period near the peak, temperature changes to within $\pm 0.1^{\circ} \mathrm{C}$ accuracy are measurable.

\section{Thermocouple Radiation Calorimeter}

To provide a reliable measurement of the total radiation from ZT-P, a time-integrated calorimeter, using a small thermocouple, was constructed. Because of the large time-varying magnetic fields near the torus, this was the only approach which could be guaranteed to work, since its measurement is made 10-1000 ms after the discharge has been terminated. A time-resolved radiation detector, which can function in the time-varying fields, will be developed in the future.

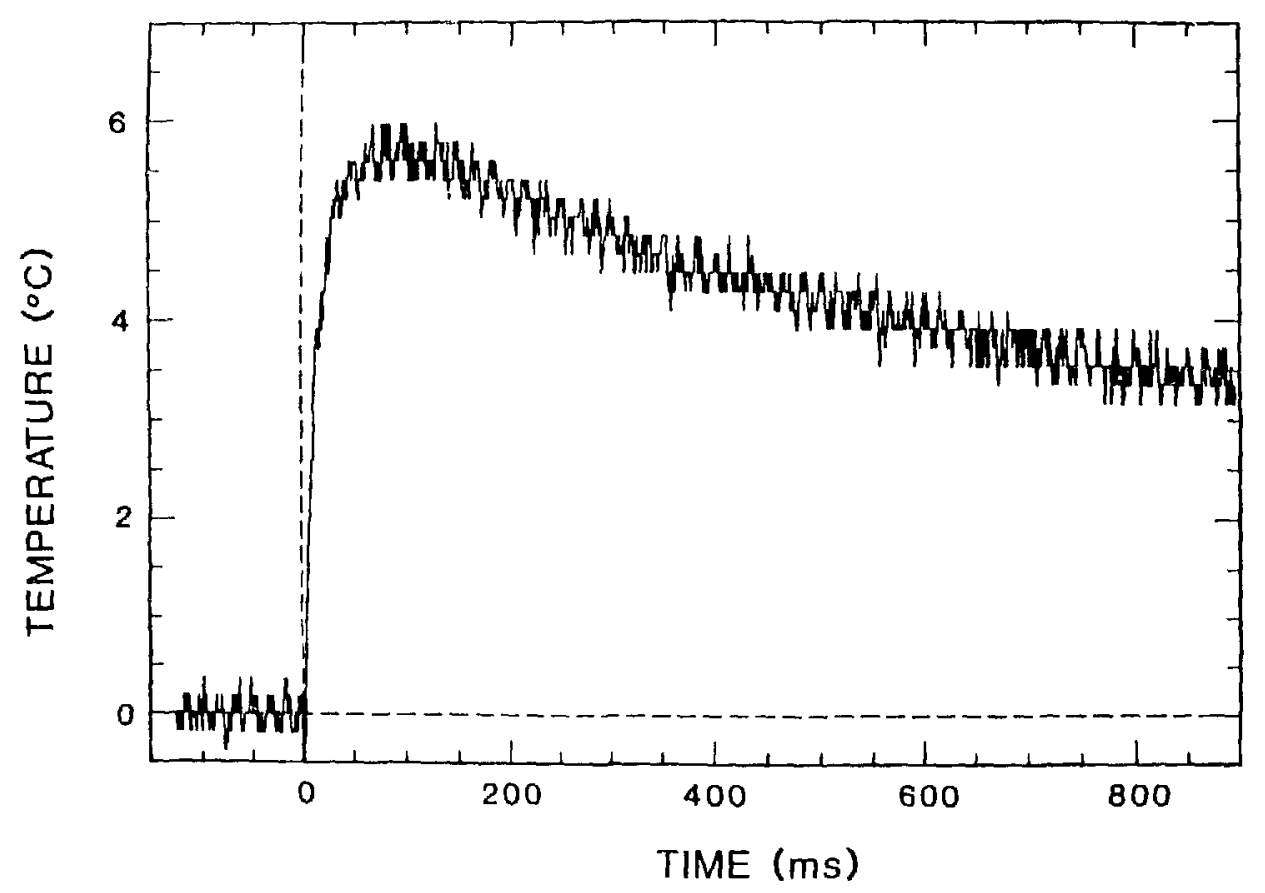

Fig. VII.3.

Temperature of a ZT-P liner thermocouple as a function of time. The plasma discharge of about 1 ms duration occurs at time zero. 
As shown in Fig. VII.4, the calorimeter consists of a $0.05-\mathrm{mm}$ stainless steel foil mounted on a tube $20 \mathrm{~mm}$ back from the end of the disgnostic tubulation. The diameter of the exposed foil disk is $2.2 \mathrm{~cm}$. To the back of the foil is soldered a Chromel-Constantan thermocouple junction (0.025-mm wire) using a minimum amount of tin-silver solder (melting point $240^{\circ} \mathrm{C}$ ). The $0.025-\mathrm{mm}$ thermocouple leads are soldered to $0.075-\mathrm{mm}$ twisted pair thermocouple wire which is supported by a Teflon pedestal and clamp. The soldering techniques used are similar to those described in the thermocouple section. Since tin has a lower vapor pressure than silver, this solder was judged suitable for use in the vacuum.

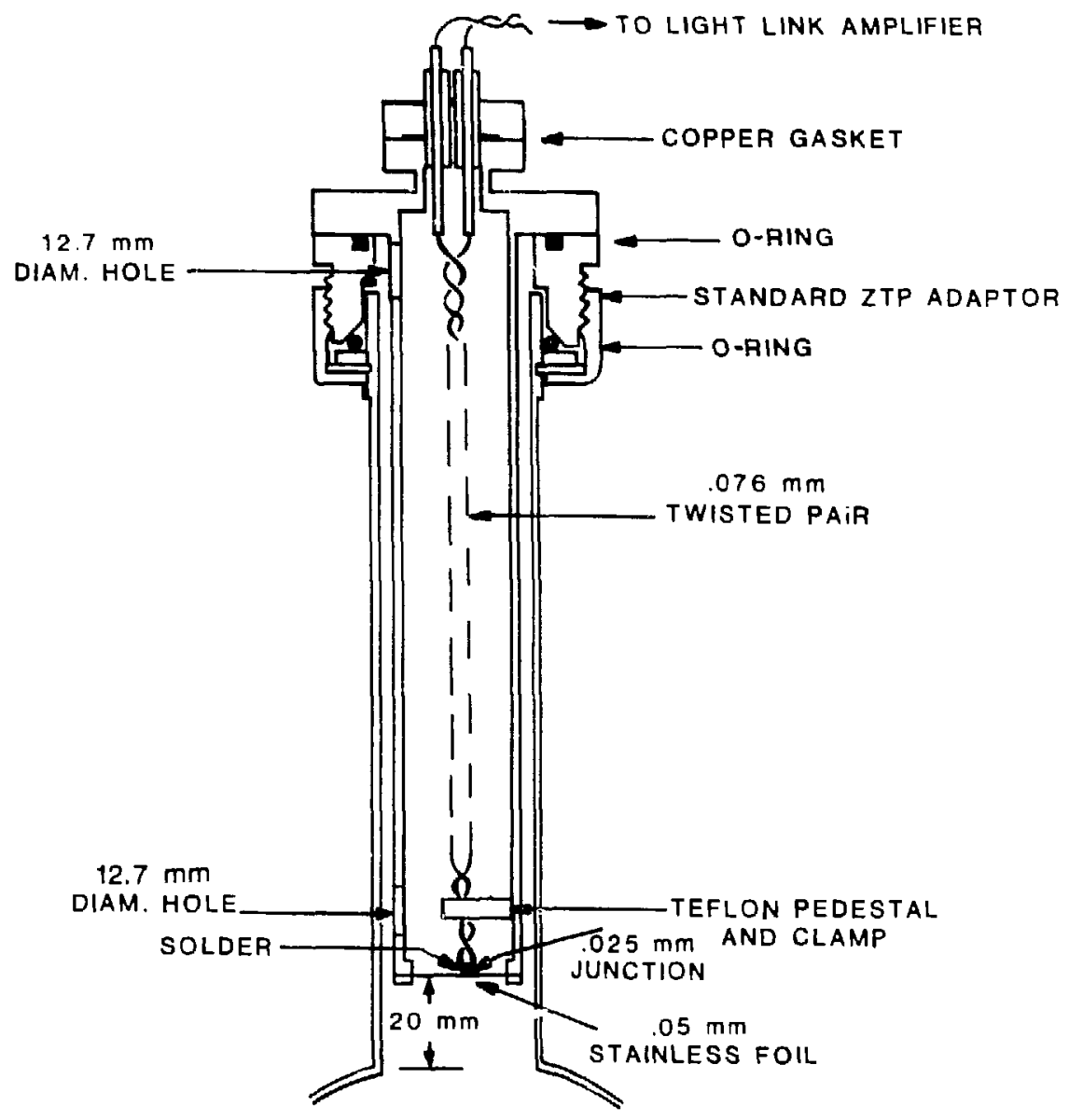

Fig. VII.4.

Schematic diagram of the thermocouple radiation calorimeter. The calorimeter absorption foil is shown recessed $20 \mathrm{~mm}$ back in the tubulation from the plasma edge. 
Two 12.7-mm diameter access holes are drilled in the support tube at a $90^{\circ}$ orientation opposite to the Teflon pedestal, and also adjacent to the vacuum feedthroughs. The twin vacuum feedthroughs are mounted in a vertical plane perpendicular to the minor axis to minimize magnetic pickup from the poloidal and radial fields.

The sensitivity of this detector is estimated to be adequate. If $20 \%$ of the energy dissipation $(=1.7 \mathrm{~kJ}$ ) of a low-current, 20-kA discharge is emitted as radiation, this would give an energy flux at the calorimeter foil of $0.011 \mathrm{~J} / \mathrm{cm}^{2}$ which results in a temperature rise of $0.55^{\circ} \mathrm{C}$. This is easily measurable using the thermocouple approach.

Visible and VUV Spectroscopy

Spectroscopic diagnostics on ZT-P are designed to measure the impurity content of the plasma, provide information on the electron temperature, and determine the recycling rate of deuterium. The low $Z$ impurity content is obtained by measuring the absolute intensity of the appropriate resonance lines of oxygen, carbon and nitrogen using a $0.2-\mathrm{m}$ vacuum ultra-violet (VUV) Acton Research Corporation spectrometer. Figure VII.5 shows the system: light emitted along a vertical diameter of ZT-P is focused by a concave osmium coated mirror onto the entrance slit of the spectrometer. The absolute calibration is established using a deuterium lamp from 350 to $121 \mathrm{~nm}$, with a lower wavelength sensitivity established by cross-calibration to a $0.4-m$ absolutely calibrated spectrometer on loan from the Johns Hopkins University.8 Branching ratio techniques for calibration can also readily be used, since an identical spectrometer, absolutely calibrated in the near $U V$ and visible, gathers light from the same vertical chord, but using the upper diagnostic port. The brightness of a measured line is related to the densities by:

$$
B_{i}=(1 / 4 \pi) \int n_{i} n_{e} Q_{i}^{e x} d l
$$

$\because$ re $\mathrm{n}_{e}$ and $\mathrm{n}_{i}$ are the electron and ion densities, respectively, and $\mathrm{Q}_{i}$ ex is the electron impact excitation rate. Assuming $0_{i}$ ex to be approximately constant, and assuming no radial dependence to tile ion density, the brightness, normalized by the diameter-averaged electron density (from the interferometer) yields the ion density. This information is then used to 


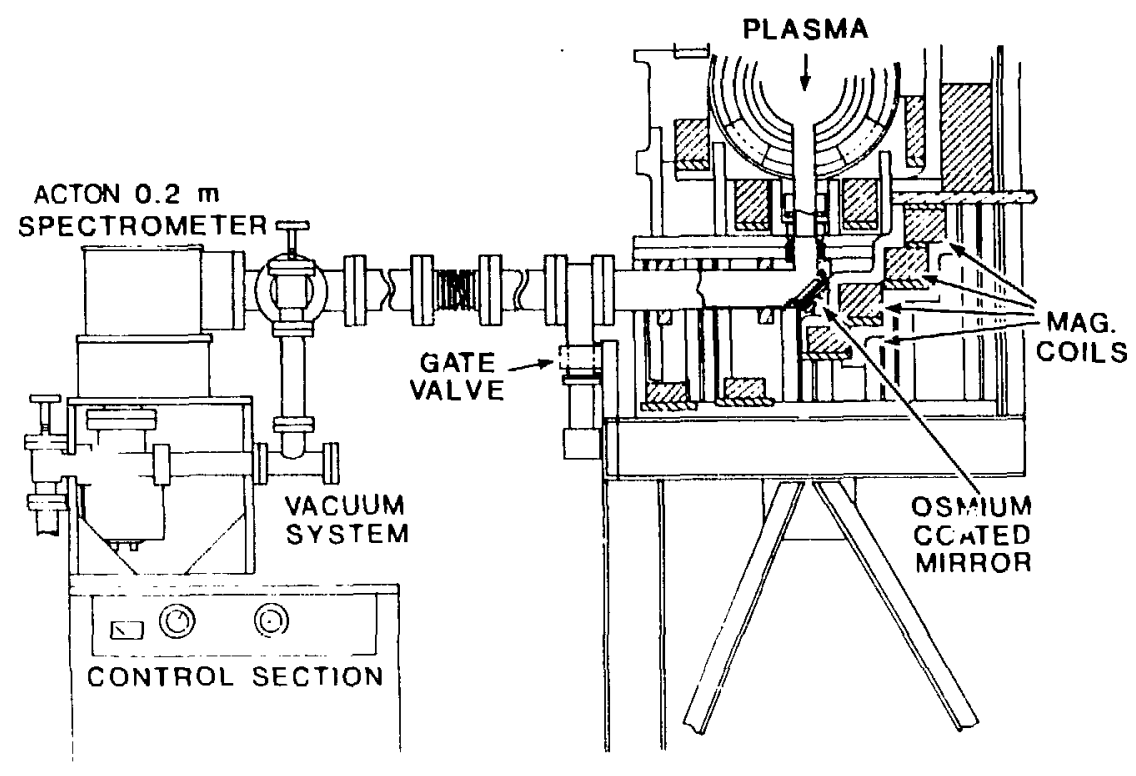

Fig. VII.5.

ZT-P VUV spectrometer system.

determine the power radiated and contribution to $\mathrm{Z}_{\text {eff }}$ by that species, using appropriate simulation codes.

The Zeff itself may be measured from the continuum radiation, using a photomultiplier with a filter centered on a line-free region in the visible spectrum. Such a diagnostic is in use on ZT-40M9 and, provided an appropriate spectral region is identified, will be used on ZT-P.

The intensity of the line radiation from helium-like species is a strong function of the electron temperature. For example, the $\mathrm{CV}$ line emission at 227- $\mathrm{nm}$ may be used to estimate the electron temperature in the 30- to $150-\mathrm{eV}$ regime,10 with similar states of heavier ions (NVI and OVII) being useful at somewhat higher temperatures. Thus one can augment the Thomson scattering $\mathrm{T}_{\mathrm{e}}(0)$ data using the $0.2-\mathrm{m}$ spectrometers.

Deuterium recycling is examined by absolutely calibrated Balmer-alpha line monitors. These detectors are photomultipliers fitted with 1-nm FWHM filters centered at $656 \mathrm{~nm}$. Fiber optics are used to couple the plasma light to these monitors, with highly collimated (1.7-degree) acceptance angles set by apertures. This measurement is therefore highly localized, and correlates well with local plasma-wall interactions. The $D_{\alpha}$ emission is related to the 
ionization rate,11 and the particle confinement time derived by normalization to the electron density, with reasonable assumftions on the density and temperature profiles.

\section{Surface-Barrier Diode Array}

A three-chord SBD (surface-barrier diode) system for the measurement of soft $\mathrm{x}$-rays, similar to a diagnostic previously constructed for $\mathrm{ZT}-40 \mathrm{M}, 12$ has been designed and implemented on ZT-P. Wide-band fiber-optic links relay the signals to the data acquisition system. The array allows central and off-axis measurements of both the soft $x$-ray flux and fluctuations, with a frequency response of up to $150 \mathrm{kHz}$.

If used without blocking filters, the SBDs respond to radiation extending from the visible to $10 \mathrm{keV},{ }^{13}$ with the high-energy limit primarily determined by the thickness $(100 \mu \mathrm{m})$ of the depletion layer in the SBD. Typically, 5- $\mu \mathrm{m}-$ thick aluminum or 12.5- $\mu \mathrm{m}$-thick beryllium foils are used as filters, giving 7 lower energy cutoff of $600 \mathrm{eV}$. This provides a diode signal of a few $\mu \mathrm{A}$ if the plasma temperature is greater than about $100 \mathrm{eV}$. For cold discharges, the SBDs can perform as wideband radiation monitors, when used witnout filters.

Figure VII.6 shows a cross section of ZT-P at the diagnostics section used by the SBDs, and by the visible and VUV spectrometers. The two off-axis tubes allow detection of $m=1$ MHD activity, while the horizontal side tubulation can be configured either with an SBD detector, or with a fast gas-puff valve. Since this station is $160^{\circ}$ away from the pump stand, it also includes the main gas feed for the continuous flow-fill system from two tubes mounted in the side of the SBD access flange.

\section{Multi-Chord Interferometer}

The three-chord infrared heterodyne interferometer is shown in Fig. VII.7. Because of the intense and rapidly changing external magnetic fields of the experiment, the interferometer must be constructed on a fiber glass or graphite-fiber optical table. Large metallic objects such as lasers are mounted far from the machine center to avoid the high ambient magnetic fields. The plasma density measurement is made at $10.6 \mathrm{\mu m}$ using a waveguide $\mathrm{CO}_{2}$ laser and room temperature $\mathrm{Hg} \mathrm{Cd} \mathrm{Te}$ detectors. A second wavelength option $(3.39 \mu \mathrm{m})$ to eliminate the effects of room vibrations may be added as conditions warrant using a germanium Bragg cell of special design. The 


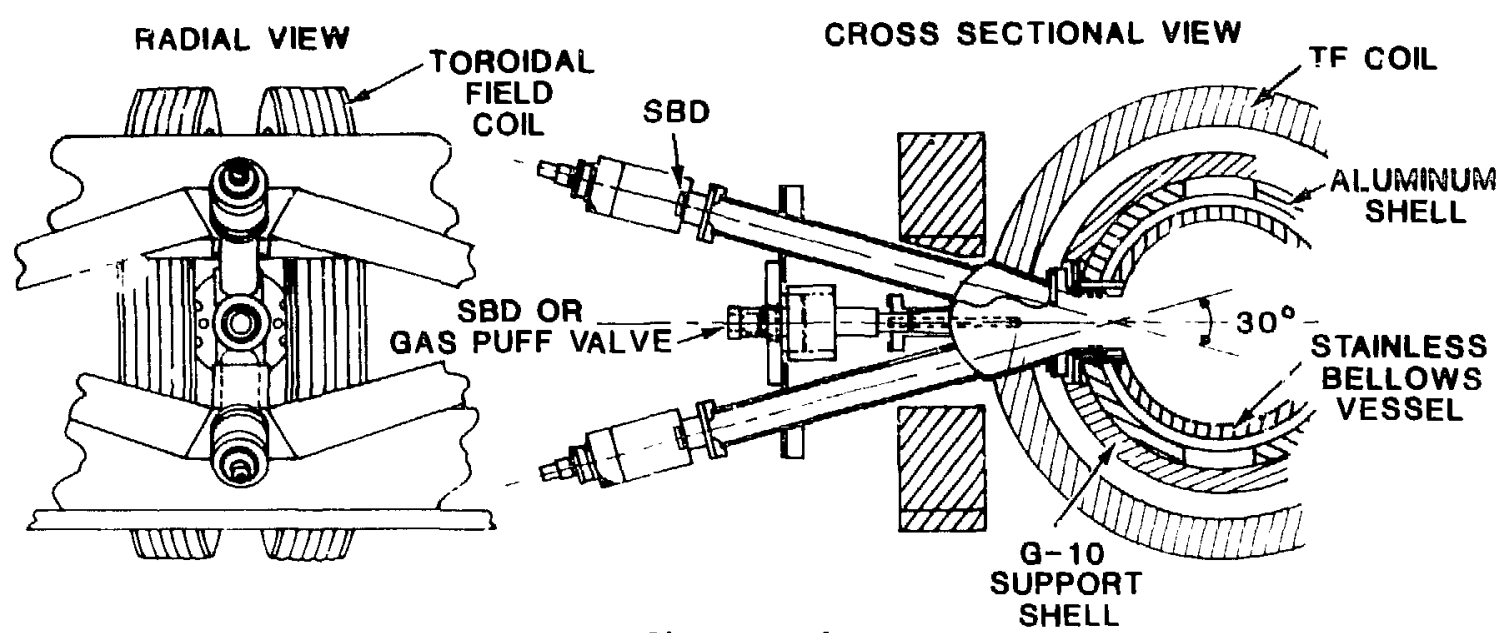

Fig. VII. 6 .

ZT-P surface barrier diode access.

interferometer design employs matched scene and reference beam path lengths and near-normal incidence beam splitters to minimize vibrational effects that cannot be corrected with a two wavelength system. Plasma density is measured as a real-time analog signal using phase detectors.

Thomson Scattering

The ZT-P Thomson scattering system is a single-pulse, single-point diagnostic. The design is similar to that of the ZT-40 system. ${ }^{14}$ The optical part of the diagnostic is drawn in Fig. VII.8.

The ruby laser and the scattered light detector are located in the experimental room. The power supply for the laser and the data acquisition system are installed in a separate area. Laser operation is controlled from the ZT-P control room by means of an optical link.

The pulsed 694.3-nm light source is a 2-stage Korad $\mathrm{K} 1500$ ruby laser. The 3/4"-diameter output beam has a nominal energy of up to $10 \mathrm{~J}$, and a rated divergence of 1.3 mradian. Measurements with a $50-\mathrm{cm}$ beam focusing leris show that $80 \%$ of the beam can pass through a 0.5 -mm-diameter pin hole.

The horizontal beam is aimed at a $90^{\circ}$ turning prismi located under the toroidal discharge chamber. The prism deflects the beam upward, and a lens focuses the laser on the major axis of the experiment. The beam enters and leaves the discharge tude through Brewster-angle windows. After leaving the torus, the beam is absorbed in a beam dump containing two blue glass plates. 


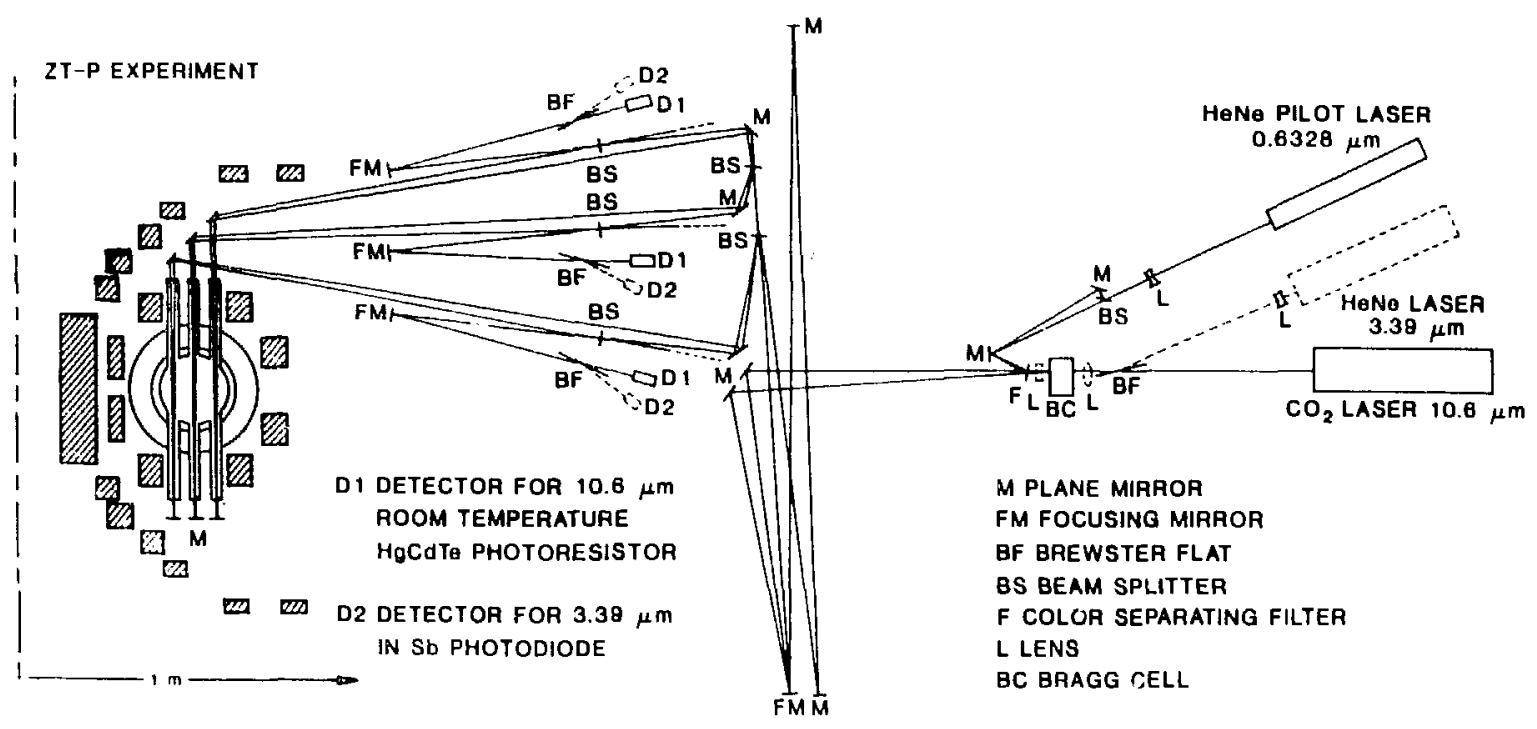

Fig. VII. 7 .

ZT-P three-chord infrared interferometer.

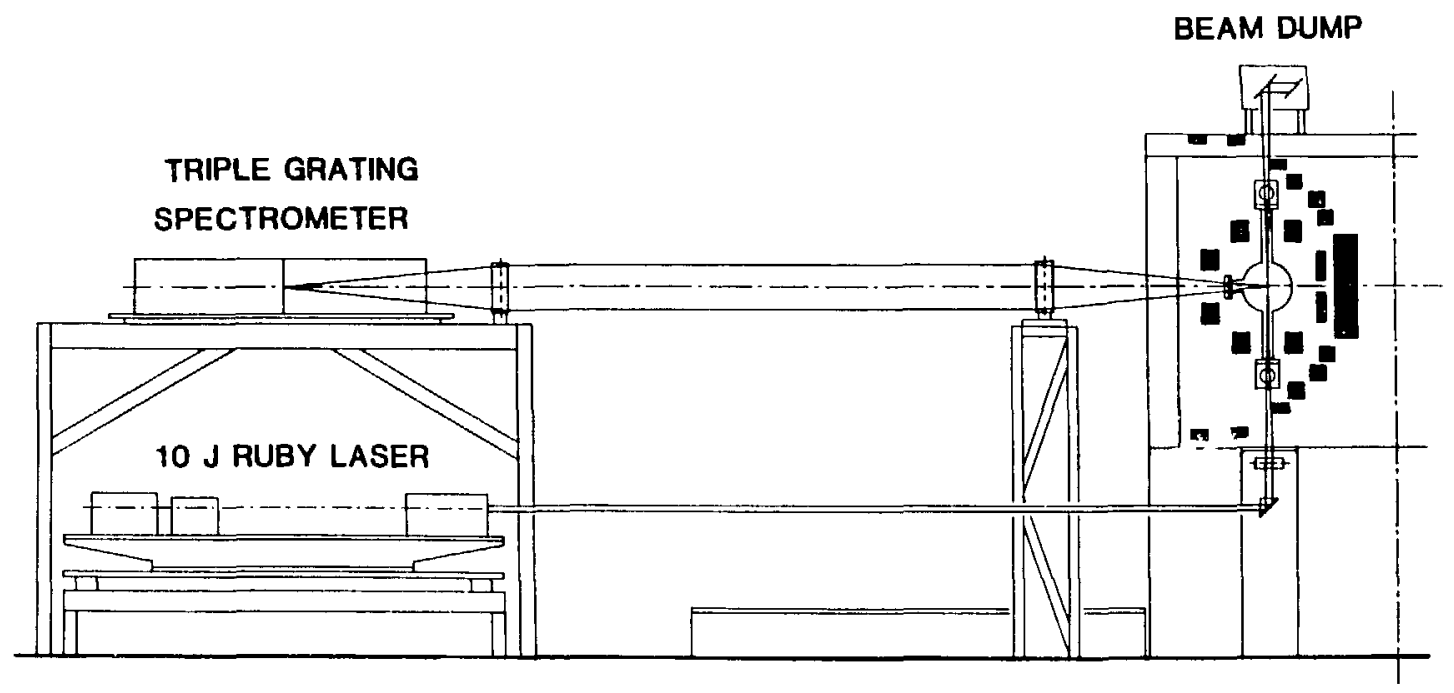

Fig. VII.8.

ZT-P Thomson scattering system. 
The scattered light is observed through a side window in the liner. The light is collimaied and focused on the entrance slit of a polychromator by two 16-cm-diameter lenses with focal lengths of $76.2 \mathrm{~cm}$.

The spectrometer is a triple-grating instrument allowing effective elimination of $694.3-\mathrm{nm}$ scattered ruby light and $656.1-\mathrm{nm} \mathrm{D}_{\alpha}$ radiation. The dispersion of this $\mathrm{f} / 5$ instrument is $2 \mathrm{~nm} / \mathrm{mm}$. The spectrally resolved light is transmitted from the exit plane to a set of up to 10 photomultipliers by means of quartz fiber guides. The photomultipliers are RCA 8852's with a rated quantum efficiency of $4.5 \%$ at $694.3 \mathrm{~nm}$. The system also accepts end-on galium arsenide tubes with a quantum efficiency of $\sim 10 \%$.

The photomultiplier signals are displayed on four dual-beam oscilloscopes during calibration and trouble-shooting. They are recorded by three LeCroy 2250L 12-channel charge-sensitive digitizers during normal operation. The signals to the digitizers are delayed by $150 \mathrm{~ns}$. The first digitizer is activated during the laser pulse, the second unit $150 \mathrm{~ns}$ after the pulse, and the third digitizer 300 ns after the pulse. The first and third digitizers provide the information necessary to subtract plasma light from the scattering signal recorded by the second digitizer.

As on ZT-40, the data are analyzed by fitting the scattering signals to a Maxwellian distribution which includes a relativistic correction. The program takes the wavelength response of the photomultiplier channels into account by performing a convolution with the calculated distribution. The ZT-P computer displays the measured electron temperature and relative electron density shortly after the plasma shot.

VIII. DATA ACOUISITION SYSTEM

Introduction

The data acquisition system collects the data produced by the ZT-P diagnostics, digitizes the signals, and makes the results available on a CAMAC highway for further processing by the ZT-P computer. Diagnostics, transient recorders and computers are located in different experimental areas. The various elements of the system are linked by analog and digital data ways.

One of the design criteria of ZT-P is that there be no hardwired connection between the machine room where the data are acquired, and the control room where data are recorded. This condition is met by using two 
different data link systems. One reason for this approach is to test different concepts for future RFP designs.

In the first system, data are transmitted from the experiment to the control room by means of optical analog links. The digitizers are located in CAMAC crates, which communicate with the ZT-P computer by means of an optical serial highway. To optimize the signal-to-noise ratio, signal conditioners are installed at the transinitter end of the analog links. This system is well suited for diagnostics such as the liner thermorouples which require electrical isolation.

In the second system, the data are conditioned and recorded at a remote CAMAC station located close to the machine. This station communicates with the ZT-P computer by means of optical serial highway. The remote CAMAC scheme is appropriate for diagnostics signals requiring a wide frequency response. Examples are the Thomson scattering and the hard x-ray signals.

Maps of the analog link and remote CAMAC installations are shown in Figs. VIII.1(a) and VIII.1(b). The analog link system handles 62 physics and engineering data channels originating in the machine room, and 19 engineering data channels from the power crowbar room. The remote CAMAC system handles 15 physics data lines from the machine room. The number of channels, in both cases, can easily be changed.

\section{Magnetic Field Considerations}

Being an air-core machine, ZT-P produces a substantial magnetic field in the experimental area. A plot of the calculated poloidal field, for a plasma current of $315 \mathrm{kA}$, is shown in Fig. VIII.2. The presence of this field affects the design of the data acquisition system in the following ways.

The magnetic field diagnostics are carefully balanced to avoid sensitivity to unwanted field components. Electronic circuitr $r_{j}=$ designed to minimize the number and size of loops that might pick up a magnetic field. Field-sensitive components, such as photomultipliers, are protected by magnetic shields. Connections to the diagnostics use shielded twisted pairs, rather than single craxial cables. The optical transmitters and associated signal conditioners are custom designed. They use differential inputs, are compact, and exhibit minimal loop areas. The locations of the optical transmitters are chosen to minimize magnetic field interference with the diagnostic leads and with the transmitter electronics. 


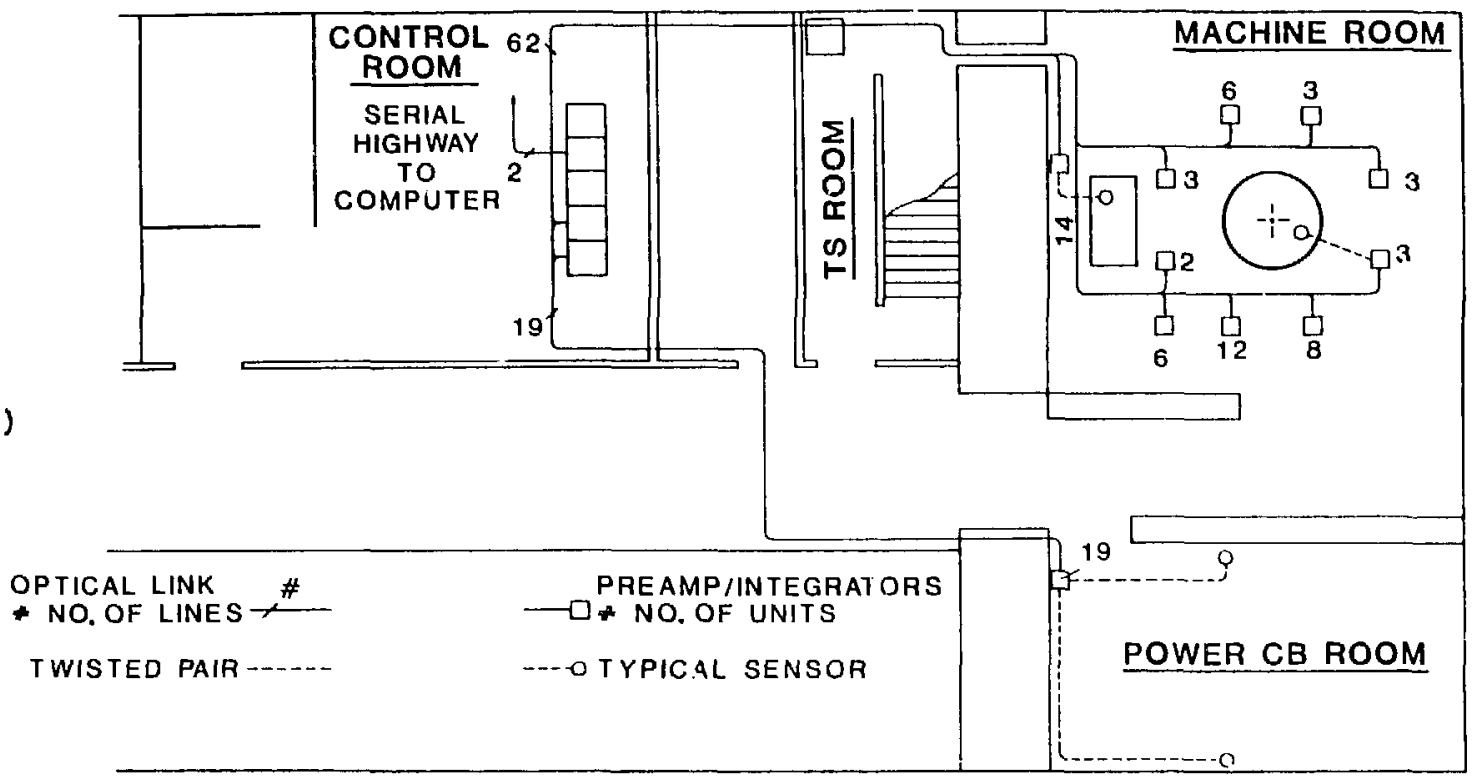

(a)

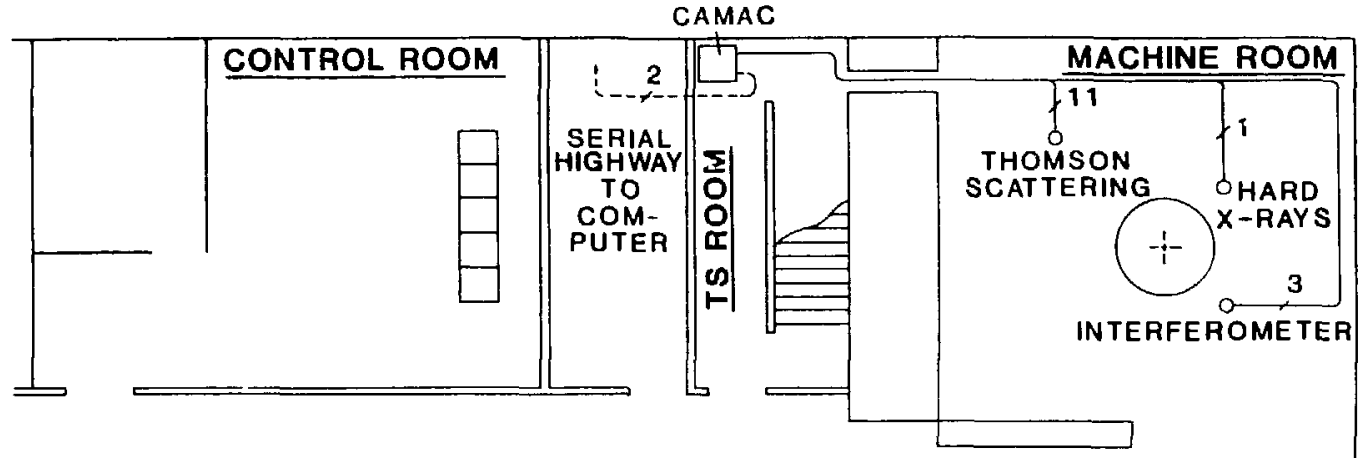

(b)

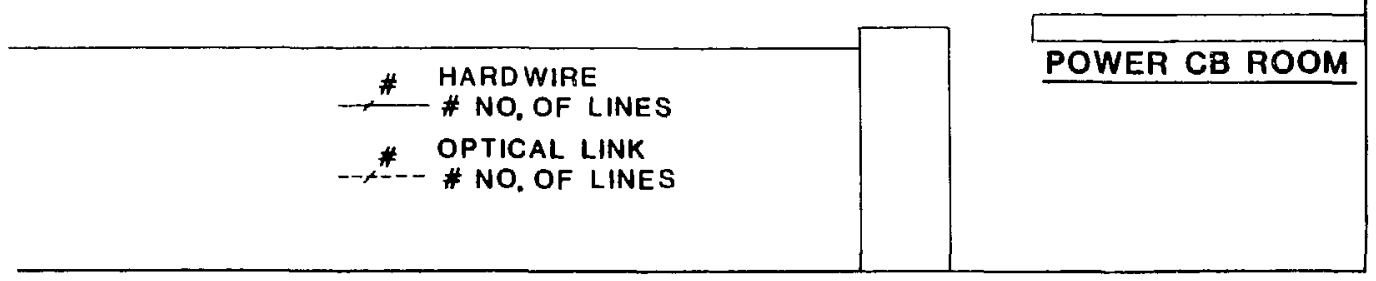

Fig. VIII. 1.

(a) ZT-P analog link system; (b) Remote CAMAC system. 


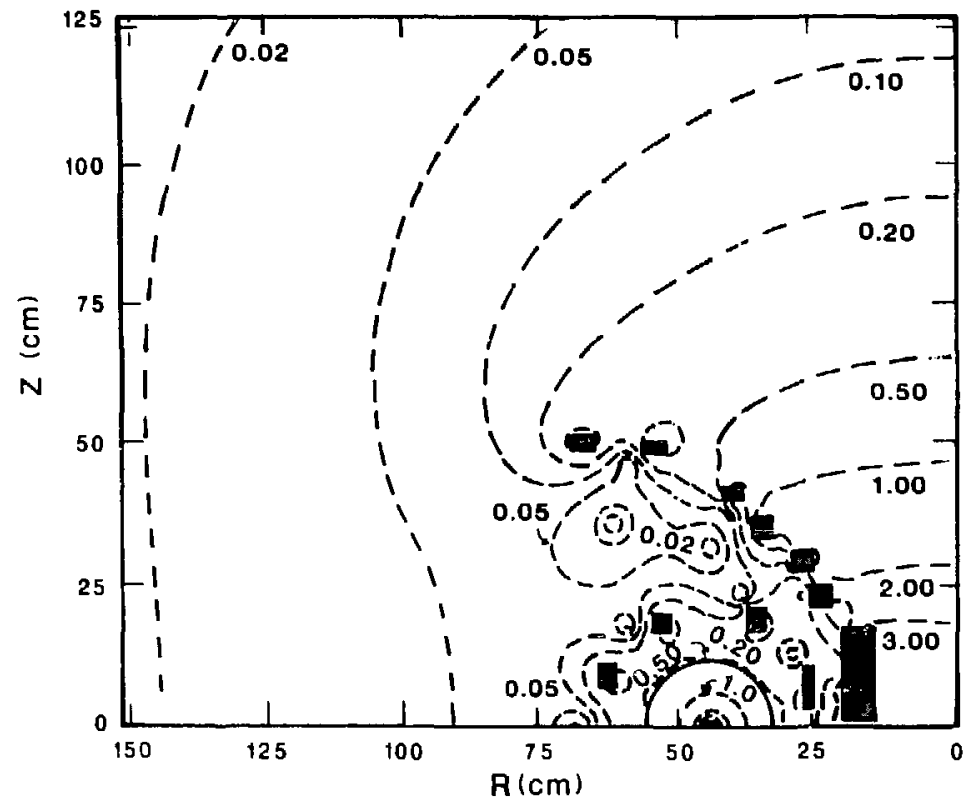

Fig. VIII.2.

Plot of Mod B contours (in Tesla) in the ZT-P experiment room for $315 \mathrm{kA}$ operation.

The remote CAMAC station uses off-the-shelf commercial components and a type of signal conditioners previously designed for a less severe environment. To reduce magnetic pick up, the remote station is locaced outside the high ambient field environment.

Care is taken with the grounding system to avoid looping stray poloidal magnetic fields. Grounding of the machine to the building ground follows the $\mathrm{AC}$ power line to the pumping station, which is in electrical contact with the vacuum vessel. As shown in Fig. VIII.3, copper bars extending radially from the center of the machine, provide grounding for the diagnostics. The potential of these bars matches the potential of the liner at corresponding toroidal locations. AC and DC supplies for the diagnostics come from wall-mounted isolation transformers and power supplies. The power leads follow the paths of the grounding system. 


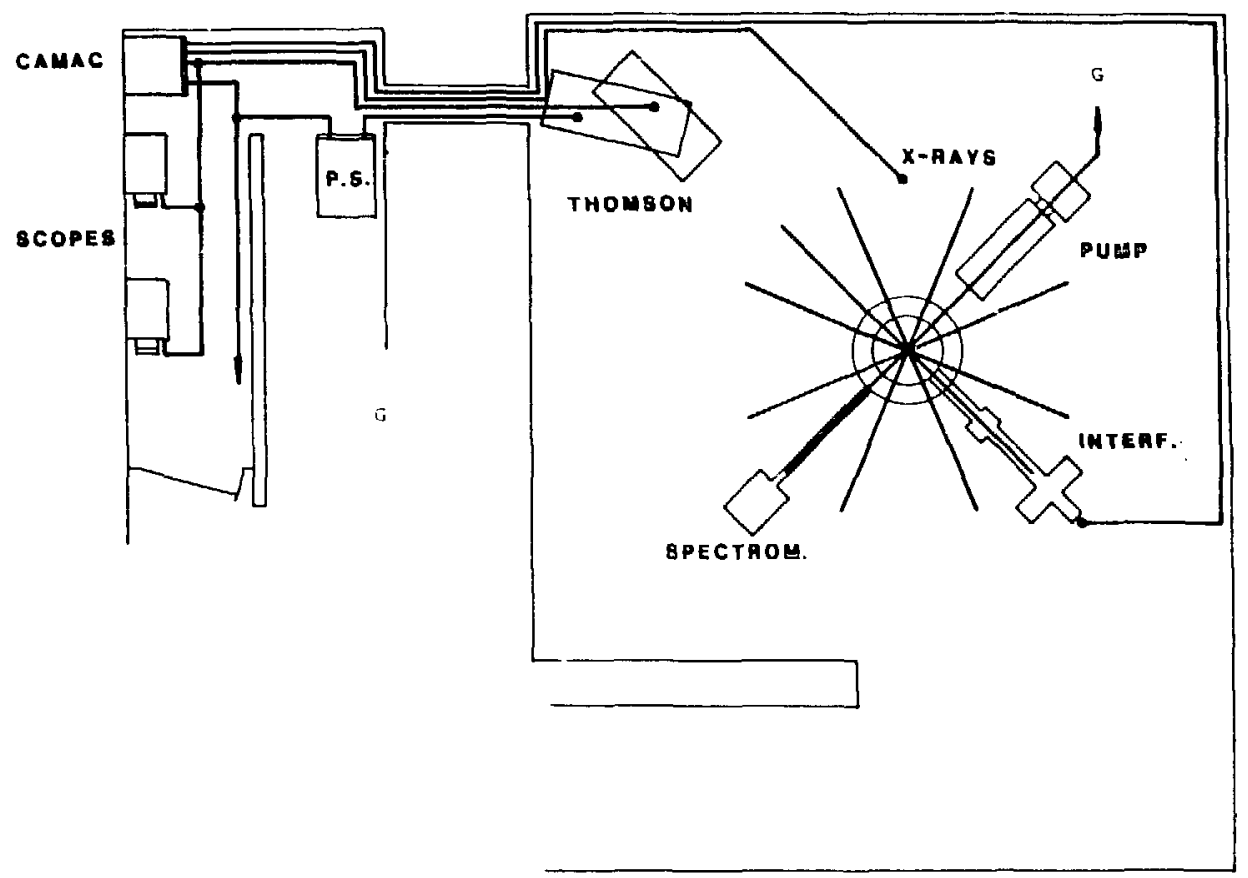

Fig. VIII. 3 .

ZT-P diagnostics grounding schenatic.

\section{Remote CAMAC System}

The remote CAMAC station is located $8 \mathrm{~m}$ away from the experiment, in the Thomson scattering power supply room. At this location, the ZT-P driven ambient magnetic field is insignificant. The $A C$ power is isolated and filtered, but there is no special electromagnetic shielding enclosure. The station includes a CAMAC crate and a NIM bin. The crate contains three LeCroy 2250L 12-channeI charge-sensitive A/D converters for Thomson scattering, and a LeCroy 8210 4-channel digitizer for the other diagnostics. The NIM bin contains signal conditioners of the type used on ZT-40 and the logic for the Thomson scattering data acquisition.

\section{Analog Link System}

Each channel of the analog link system consists of a signal conditioner, an optical transmitter, a length of optical fiber, and an optical receiver followed by a transient digitizer. The signal conditioner and optical transmitter are designed as replaceable plug-in units. They are mounted in small aluminum enclosures with $\mathrm{DC} / \mathrm{DC}$ converters supplying power to the 
cirauits. The units handling the front-end diagnostics are located under a subfloor, at a radius of $1.8 \mathrm{~m}$ from the center of the machine. The other units are mounted on the walls of the ZT-P and power crowbar rooms.

The fiberoptic cables are Eotec 471001 cables with $0.2-m m$-diameter clad glass core. The length of each cable is approximately $30 \mathrm{~m}$. The optical receivers are mounted in groups of four in CAMAC plug-in units. The receivers and transient digitizers are installed in CAMAC crates located in the ZT-P control room.

The converters are 4-channel 10-bit LeCroy 8210 s with a bandwidth of $5 \mathrm{MHz}$, and a 32-channel 12-bit LeCroy 8212 with a bandwidth of 50-kHz, used for the slow thermocouple and calorimeter signals. A listing of the ZT-P diagnostic data links is given in Table 2. The signal conditioner input configurations are discussed at the end of this section.

Analog Link Design

The analog link design is based on an in-house development by D. Trudell. The links are frequency modulated, and the carrier frequency is $500 \mathrm{kHz}$. The analog bandwidth is 0 to $22 \mathrm{kHz}$. Specifications are listed in Table 3 .

The transmitter includes a Burr-Brown VFC62 V/F (voltage-frequency) converter, and a Burr-Brown FOT110 optical transmitter. The receiver includes a Burr-Brown FOR110 optical receiver, and a VFC62 used as an F/V converter. The carrier frequency is removed with a passive 6-pole Butterworth low-pass filter. A 50- $\Omega$ line driver generates an output signals compatible with the input capability of the transient recorders. The time-delay of $20 \mu \mathrm{s}$ produced by the low-pass filter must ve kept in mind when comparing signals transmitted through the light links with signals recorded at the remote CAMAC station.

The Burr-Brown optical components have recently been discontinued. Hewlett-Packard type HFBR-1202 and HFBR-2204 transmitter-receivers are being presently used, with no apparent change in performance. The DC/DC converter, which makes the unit electrically floating, is a Burr-Brown 722 with a nominal isolation voltage of $5 \mathrm{kV}$. The converter is located in a separate compartment of the transmitter enclosure. 
TABLE 2

Typical Data Link Configuration

Diagnostic

Zink

Type

Conditioner

Number of

Type

Channels

Physics:

Tor. Voltage Loops, aver.

Tor. Voltage Loops, dift.

Tor. Crt. Rogowski

Tor. Flux Loops

Tor. Field Part. Rogowskis

Rad. Flux ai Poloidal Gap

Rad. Flux at Toroidal Gap

Rad. Flux at Tubulation

Tor. Field Sine-Cosine

Pol. Field Sine-Cosine

Pol. Coil Current Sensors

Soft X-Rays

Spectroscopy

Thermocouples

Calorimeter

Optical

Optical

Optical

Optical

optical

optical

Optical

optical

Optical

Optical

Optical

Optical (*) none 3

Optical

Optical

Optical

Hard X-Rays

Interferometer

CAMAC

CAMAC

Thomson Scattering

CAMAC

$\begin{array}{ll}2 & 2 \\ 2 & 2 \\ 1 & 1 \\ 1 & 2 \\ 1 & 2 \\ 1 & 2 \\ 1 & 0 \\ 1 & 0 \\ 3 & 8 \\ 3 & 0 \\ 1 & 6\end{array}$

Engineering :

Roving

Optical

1

8

Tor. Field Current

Optical

Optical

1

5

Tor. Field Voltage

Optical

Pol. Field Current

Pol. Field Voltage

Optical

$\mathrm{ZT}-40$

none

none

3

11

Link Development:

Roving

Optical

1

1

(*) The soft $x$-ray diagnostic uses a special link with a $6-\mathrm{Hz}$ to $6-\mathrm{MHz}$ bandwidth. This AM link, developed by R. Hall, contains an Optelecom transmitter/receiver pair. 
TABLE 3

\section{Analog Optical Link Specifications}

$\begin{array}{lll}\text { Gain } & 1.0 & \\ \text { Bandwidth } & 0-22 \mathrm{kHz} & \mathrm{V}_{\text {in }}=0.1,1 \text { or } 10 \mathrm{Vpp} \\ \text { Rise time } & 15 \mathrm{\mu s} & \mathrm{R}_{\mathrm{L}}=50 \Omega \text { or } 1 \mathrm{M} \Omega \\ \text { Delay } & 20 \mathrm{\mu s} & \\ \mathrm{V}_{\text {out }} & 10 \mathrm{Vpp} & \begin{array}{l}\text { offset } \pm 5 \mathrm{~V} \\ \mathrm{R}_{\mathrm{L}}=50 \Omega\end{array} \\ \mathrm{R}_{\text {in }} & -50 \mathrm{k} \Omega & \\ \mathrm{R}_{\text {out }} & <100 \mathrm{~m} \Omega & \\ \text { Harmonics } & <0.5 \% & \begin{array}{l}\text { static measurement } \\ \mathrm{V}_{\text {out }}=10 \mathrm{Vpp}\end{array}\end{array}$

\section{Signal Conditioner Design}

The signal conditioner contains two 0p-Amp stages. The gain of the second stage can be varied from one to eleven by means of a 10-position switch. The first stage is normally wired as an amplifier with a gain of 1 or 100 , or as an integrator with an RC time constant of 0.2 or $1.0 \mathrm{~ms}$, and a DC gain of 100. These values are typical, and can easily be changed at the request of the user. The offset of the output voltage of the preamplifier can be varied to make best use of the dynamic range of the optical link. The input of the conditioner is differential, and normally includes an RC low-pass filter with a cut-off frequency of $63 \mathrm{kHz}$. The unit is assembled on a printed circuit board which plugs in the master board of the optical transmitter. Specifications are listed in Table 4.

The conditioner uses one of three input configurations. The type-1 configuration accommodates a single input channel, and is used for most electrical and optical diagnostics. The type-2 configuration is used for the measurement of the toroidal loop voltage. It consists of a resistive network and of two preamplifiers and two transmitters. The two outputs correspond to the average and difference of two loop voltages. The difference signal is time-integrated, and is used to determine the global equilibrium position of 
TABLE 4

Signal Conditioner Specifications ${ }^{a}$

$v_{\text {out }}$

$\pm 12 \mathrm{~V}$

RMS output noise

$0.02 \mathrm{mV}$

1st stage gain of 1

$5 \mathrm{mV}$

100

$0.2 \mathrm{mV}$

$0.05 \mathrm{nIV}$

$\underset{\text { 1st stage }}{\mathrm{RC}}$ of $\underset{1}{0.2 \mathrm{~ms}}$

Common mode

rejection ratio

$120 \mathrm{~dB} / 50 \mathrm{~Hz}$

$92 \mathrm{~dB} / 20 \mathrm{kHz}$

1st stage gain of 1

$86 \mathrm{~dB} / 50 \mathrm{~Hz}$

1st stage gain of 100

$78 \mathrm{~dB} / 20 \mathrm{kHz}$

$94 \mathrm{~dB} / 50 \mathrm{~Hz}$

1st stage RC of $0.2 \mathrm{~ms}$

$52 \mathrm{~dB} / 20 \mathrm{kHz}$

$79 \mathrm{~dB} / 50 \mathrm{~Hz}$

$68 \mathrm{~dB} / 20 \mathrm{kHz}$

1st stage $\mathrm{RC}$ of $1.0 \mathrm{~ms}$

$\overline{{ }^{a} \text { Second stage gain }}$ set at 10 , in all above cases.

the discharge. A circuit diagram of the mixing network is shown in Fig. VIII.4.

The type- 3 configuration is used to handle the mode coil signals. These coils are used to measure the $m=0$ and $m=1$ components of the poloidal distribution of the magnetic field at the inner surface of the shell. Signals from four mode coils are combined resistively before being applied to two preamplifiers, where they are time-integrated and sent to two optical transmitters. The two output channels give the sine and cosine components of the field distribution. The $m=0$ or $m=1$ mode is selected by changing the resistors in the network shown in Fig. VIII.5. 


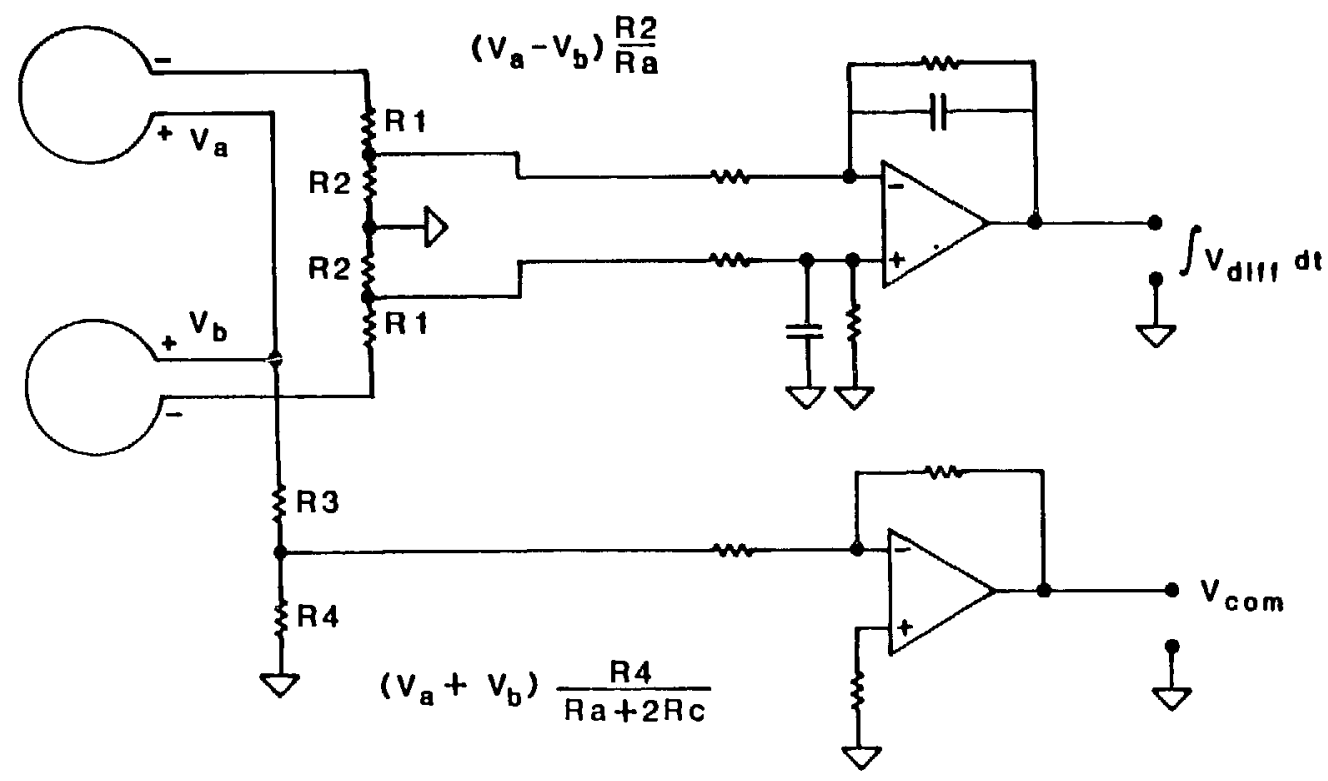

$\mathrm{Ra}=\mathrm{R} 1+\mathrm{R} 2 ; \mathrm{Rc}=\mathrm{R3}+\mathrm{R4}$

Fig. VIII.4.

Voltage loop mixing network for ZT-P. The signal sum is used to compute the loop voltage, whereas the time-integrated signal difference provides the global equilibrium position of the discharge.

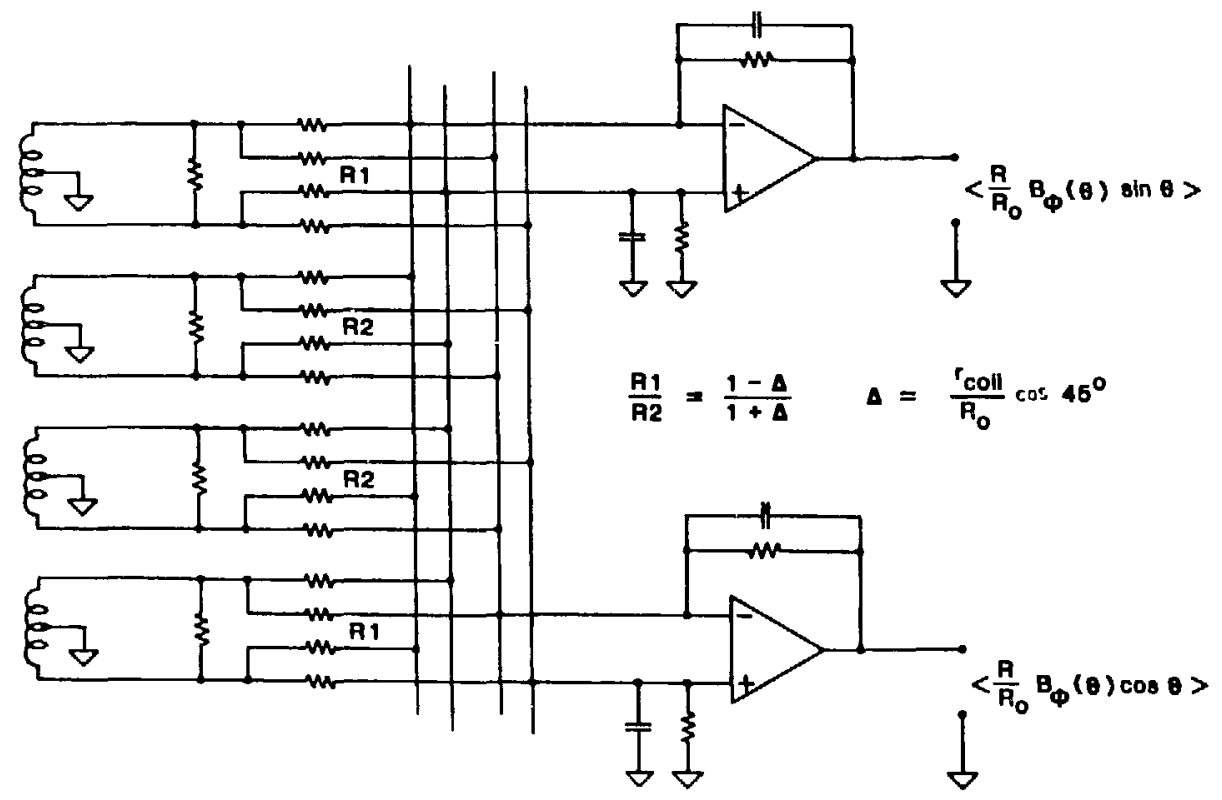

Fig. VIII.5.

Mode coil mixing network for ZT-P. 
IX. CONTROL SYSTEM

A photograph of the ZT-P control center is shown in Fig. IX.1. ZT-P uses - . a fiber optically coupled system for machine control. The control computer is linked to the control center via a fiber-optic byte-serial CAMAC highway and the control signals are fiber optically coupled to the machine transducers. This arrangement isolates electrically noisy machine areas from the sensitive electronics in CAMAC and the control computer. A schematic of the ZT-P control system is illustrated in Fig. IX.2.

The ZT-P control computer is a Prime 400 with 1 megabyte of main memory. Control hardware consists of a BIRA Branch Driver and a parallel CAMAC highway which has access to the two byte-serial CAMAC highways that span the 120 meters from the computer screen room to the ZT-P control area. The computer exercises control on the charge, vacuum, interlock, and timing systems of the machine. The charge and vacuum systems are controlled by four classes of signals: digital-out, digital-in, analog-out, and analog-in. The interlock systems consists of digital-in signals, a watchdog timer, the control panel, and an interlock chassis. The timing system consists of programmable delay generators, laser trigger chassis, and confirm circuits which notify the digital-in modules.

The digital-out class of signal (Fig. IX.3) originates in a BIRA 3222 CAMAC module. This low-true digital signal is coupled from the control CAMAC module via a 40 conductor cable to the optics CAMAC module, where it is converted to an optical signal. This signal activates a solid state relay which controls the machine component. The solid state relay is a Teledyne model $611-2$, which is a 25 -amp, 240-volt relay modified to accept an optical input.

The digital-in class of signal (Fig. IX.4) originates in an optical source CAMAC module. This optical signal is routed through the optic machine switch and sent to the digital-in optics module. The signal is now converted to a low-true digital signal and coupled via a 40 conductor cable to a Standard Engineering model PR604 digital-in module. The signal, which reflects a machine state, is read from the controlled CAMAC crate by the control computer. 


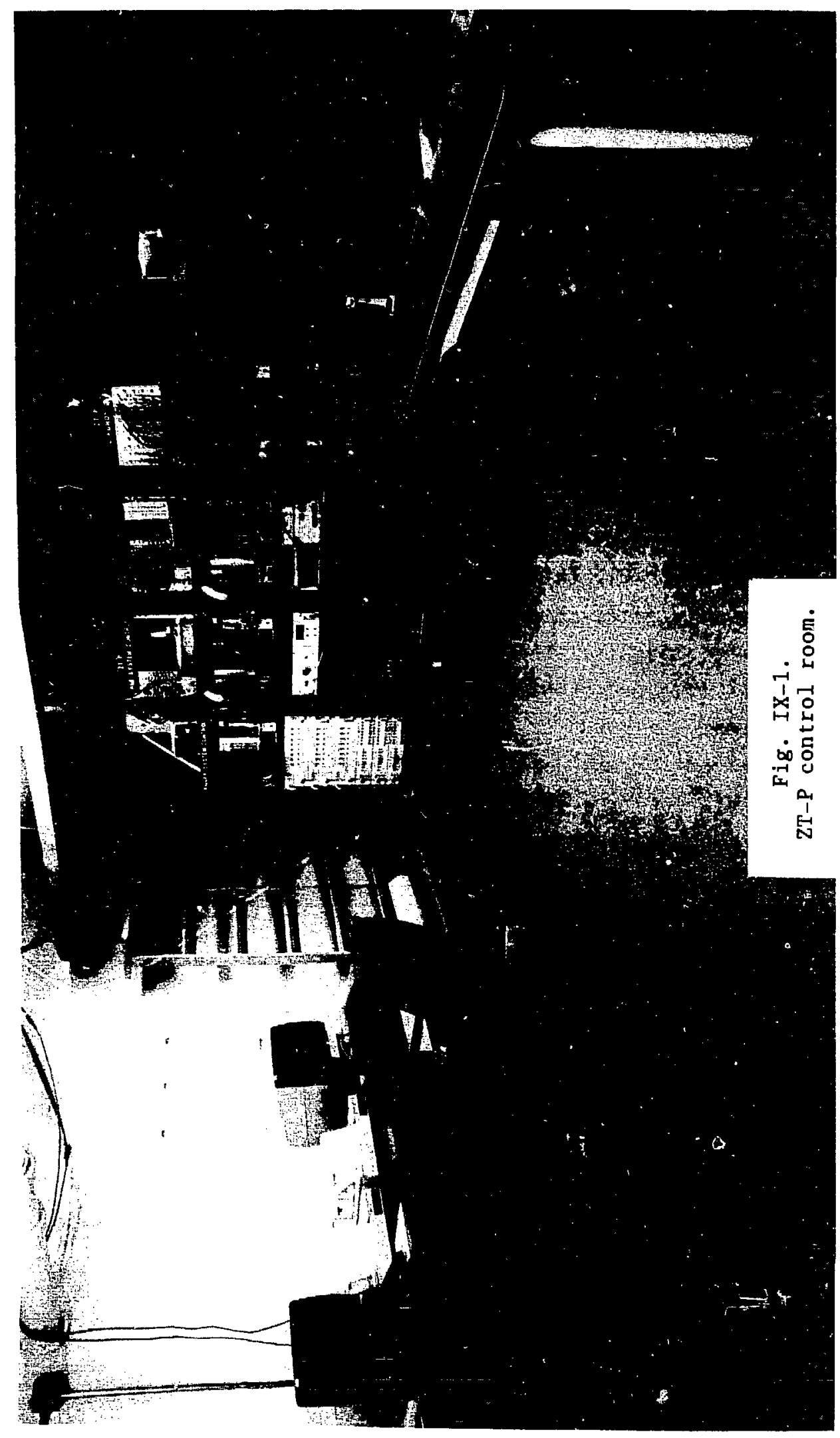




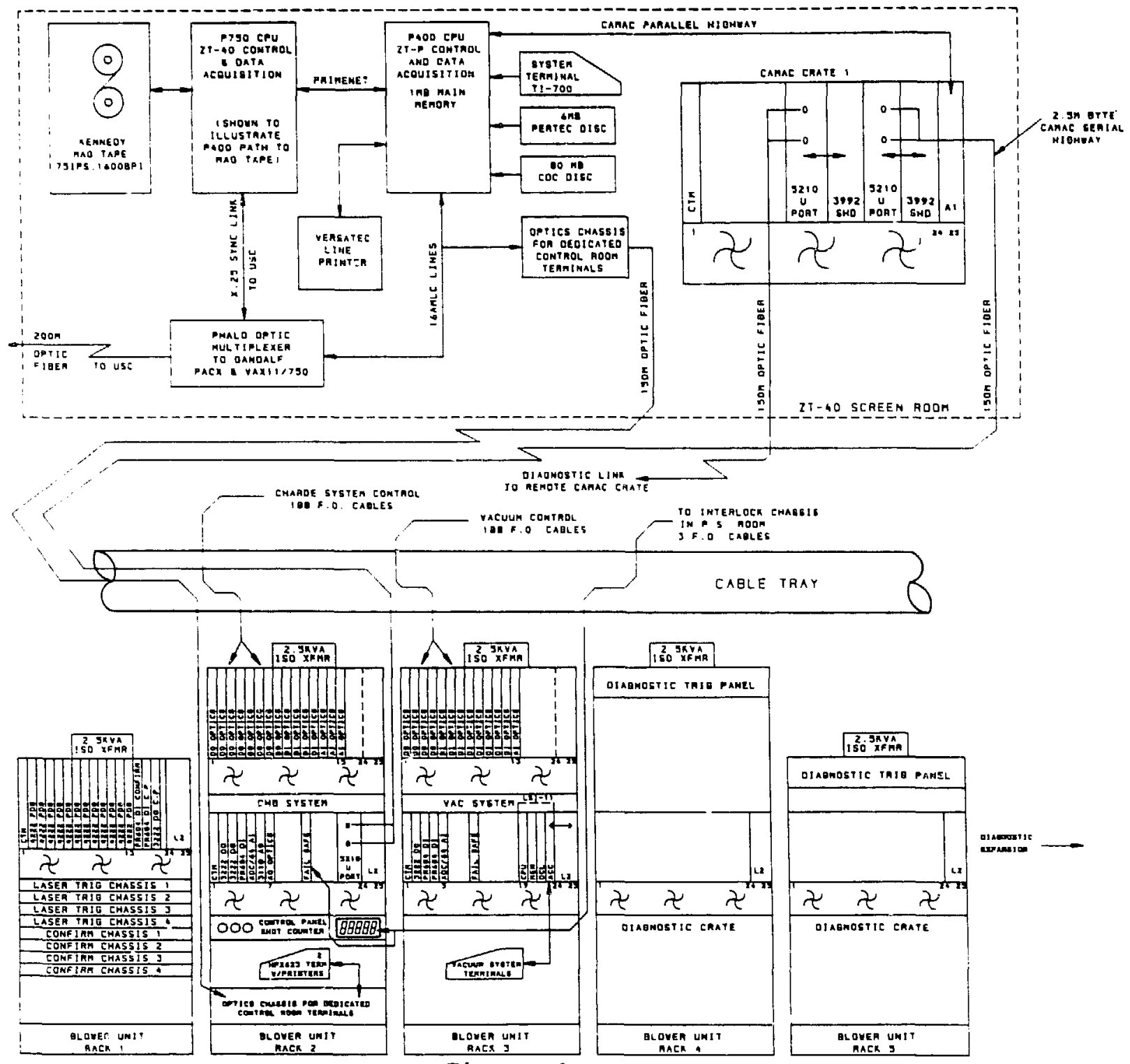

Fig. IX. 2.

ZT-P control system schematic.

The analog-out class of signal (Fig. IX.5) originates in a Kinetic Systems modei 3110 digital to analog converter. The output has a 10-bit resolution and a settling time of $1 \mathrm{~ms}$. The output signal of $0-10$ volts is routed to the analog output optics module, where it is converted to an optical pulse train of varying f.requency and shipped to the analog-out receiver over a fiber-optic cable. A pulse train of $1 \mathrm{kHz}$ represents zero volts and a train of $100-\mathrm{kHz}$ pulses represents a full scale reading of $10-\mathrm{V}$. The analog-out 


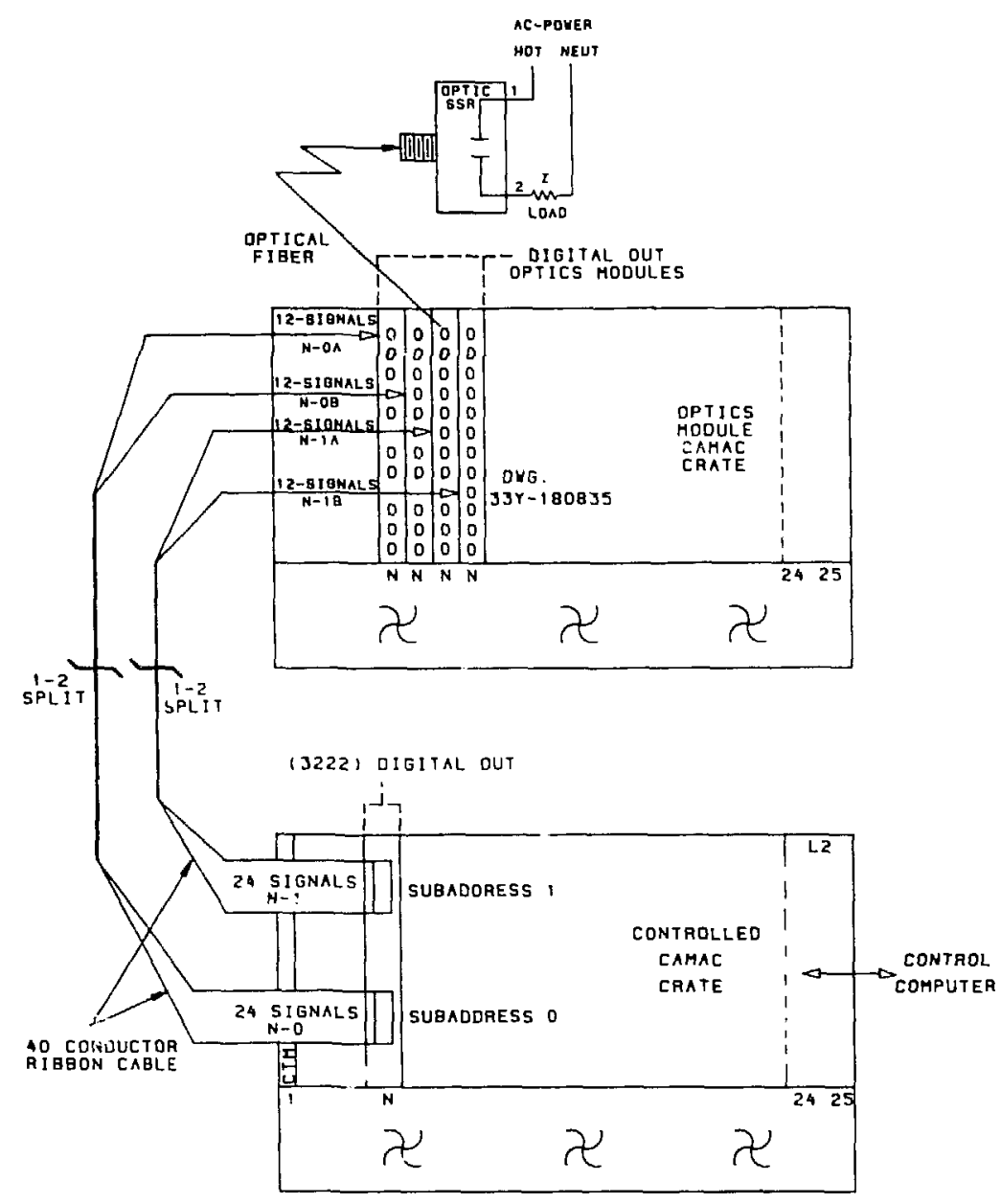

Fig. IX. 3.

Digital-out module schematic.

receiver performs frequency-to-voltage conversion and the 0-10 volt analog signal is available for machine actuators.

The analog-in class of signal (Fig. IX.6) originates at the machine as a 0-1 ma signal. The signal is converted to an optical pulse train of varying frequency and shipped to the analog-in optics module. Frequency-to-voltage conversion is performed on the signal and the signal is routed to an Interface Standards ADC/64 analog-to-digital converter. The ADC/64 performs 12-bit conversion on the $0-10$ volt analog signal and allows the control computer to read the machine signal from the controlled CAMAC crate. 


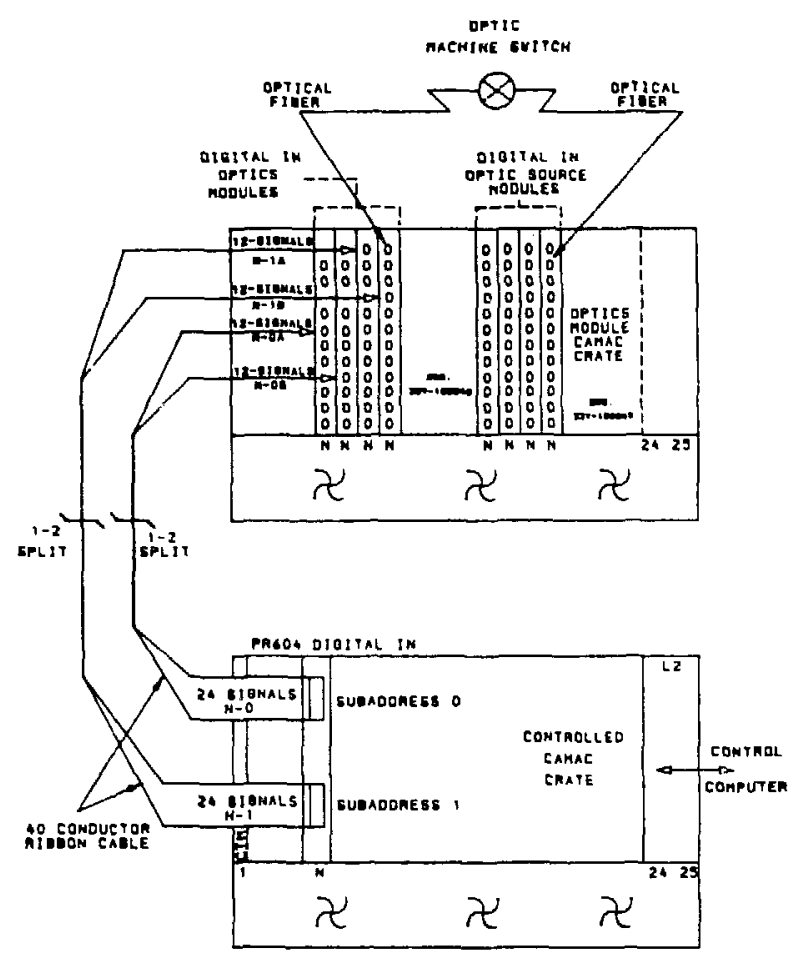

Fig. IX.4.

Digital-in module schematic.

The interlock system analyzes critical machine signals of the digital-in class. These signals are typically doors, safety chains, pressure switches, scrams, and fail-safe modules which reflect possible personnel or machine safety hazards. The hardware that performs this function consists of a digital-in system, which allows the computer to read the status; an interlock chassis, which interrupts power if any signal in the critical chain is broken; an operator control panel, which has access to the critical chain of signals; and a fail-safe module, which contains a watchdog timer updated by the control computer.

Machine timing signals are generated by the trigger system which consists of 24 channels of LRS 4222 programmable delay generators. These generators are programmable for delays up to $16.7 \mathrm{~ms}$ in one ns steps. The control computer sets up the delay channels and initiates a machine "shot" by issuing a timing pulse. The delay channels pulse the laser trigger chassis and an optical pulse fires the high-voltage trigger system on the machine. Confirm 


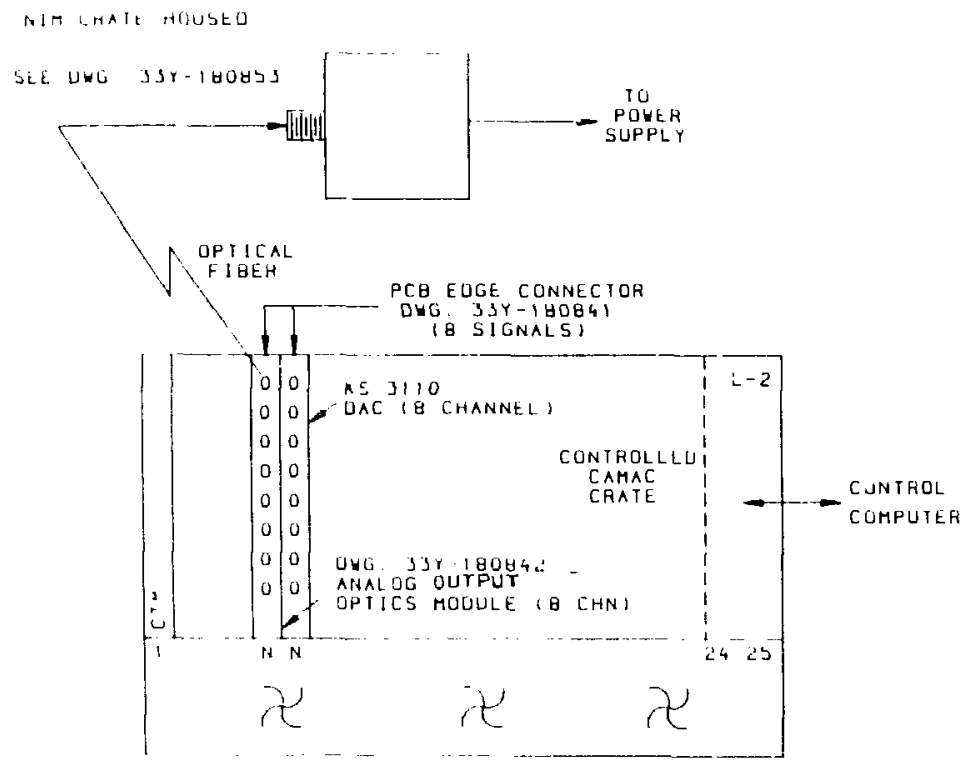

Fig. IX. 5.

Analog-out module schematic.

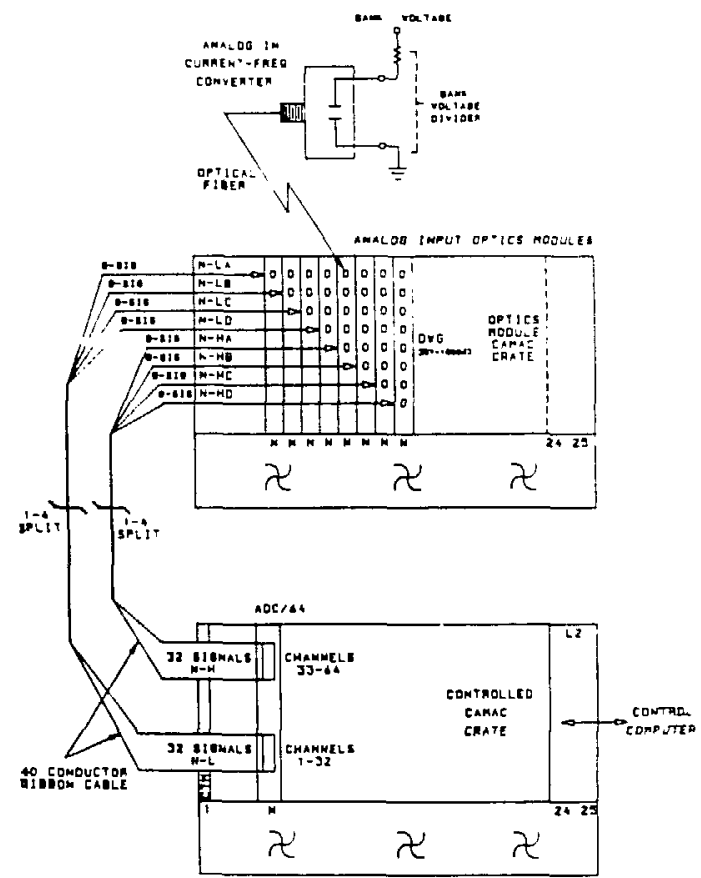

Fig. IX. 6.

Analog-in module schematic. 
chassis detect the high-voltage trigger and notify the computer via a digital-in module.

The ZT-P computer control design has several advantages in addition to the isolation afforded by the fiber-optic coupling. Since only fiber optic cable enters the control room, no hazardous voltages can be encountered due to a miswire or machine fault condition. The compact design of the fiber-optic control system allows approximately 520 signals for ZT-P's four CAMAC crate/two equipment rack implementation (Fig. IX.1). This maximum capacity is about three times the number of signals, presently required to control the ZT-P machine. Per-channel costs for the fiber optic design are approximately the same as per-channel costs for a wire design. Since the per-channel costs are dominated by the fiber optic cable, cost reductions will be observed as less expensive fiber opric cable becomes available.

\section{ZT-P PERFORMANCE - PRELIMINARY RESULTS}

This section illustrates several examples of initial ZT-P operation with and without power crowbar sustainment. Its purpose is to demonstrate that the machine design previously described produces reasonable RFP discharges, and is therefore capable of executing its programmatic research. A report of detailed engineering and physics measurements is left for future work.

At the time the data discussed in this section were obtained, all diagnostics were operaticnal with the exception of Thomson scattering and interferometry. In addition, the equilibrium feedback amplifiers were still under construction and hence the applied equilibrium vertical field was controlled by passive programming. The lack of dynamic equilibrium contrul ultimately limited the achievable discharge lifetime.

\section{Round-Top 0peration}

Figure X.1 displays data from a 60-kA round-top discharge. Following breakdown, which is facilitated by a thermionic tungsten filsment, the current rises to $60 \mathrm{kA}$ in $400 \mu \mathrm{s}$ with a total duration of $650 \mu \mathrm{s}$. The Fig. X.1 discharge employed a ramped startup technique where a small toroidal bias field was initially applied before the discharge initiation. After initiation, the current in the toroidal field coils was rapidly reversed, which formed a low current (15 kA) RFP. The RFP was then ramped up in current by a steady application of toroidal voltage. The mean toroidal field 
[Fig. X.1(c)] also increased by a factor of 5 during the ramp, which demonstrated the existence of a robust plasma dynamo.15 Ramped startup is an important technique for future large RFP experiments because it potentially reduces the single turn voltage and startup equilibrium power requirements over other startup techniques.

During a discharge, global equilibrium in ZT-P is provided by the thick aluminum shell. However, an improperly applied vertical field due to an incorrect current distribution in the equilibrium windings will result in a substantial field error at the shell's poloidal flux gap. This error manifests itself as a local displacement of the outer plasma flux surfaces in the region of the gap. ${ }^{16}$ Figures $X .1(\mathrm{~g})$ and $X .1(\mathrm{~h})$ plot these radial and vertical displacements. Preliminary data show a strong correlation between discharge termination and a large local displacement. Discharge lifetime is, therefore, dependent on good equilibrium control at the gap.
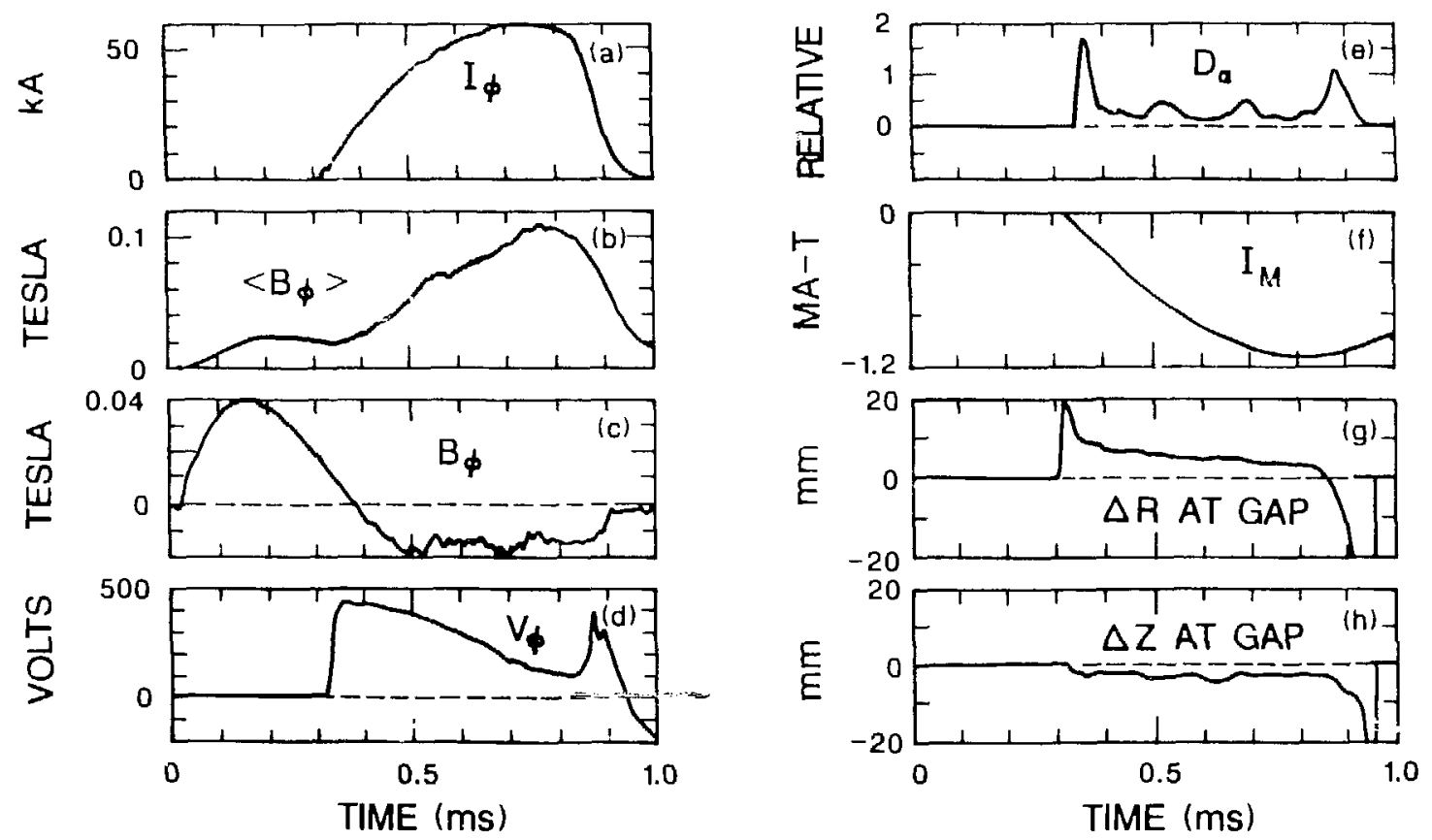

Fig. X.1.

Condensed parameters for a 60-kA round-top discharge: (a) Toroidal plasma current, (b) Volume averaged toroidal magnetic field, (c) Toroidal magnetic field at the plasma edge, (d) Toroidal loop voltage, (e) $D_{\alpha}$ emission, (f) Magnetizing current in MA-turns, (g) outer flux surface radial displacement at the gap, (h) Vertical displizement at the gap. 
Figure X.2 plots the time history of the discharge in terms of the dimensionless parameters $F\left[F=B_{z}(a, t) /\left\langle B_{z}\right\rangle\right]$ and $\theta\left[\theta \equiv B_{\theta}(a, t) /\left\langle B_{z}\right\rangle\right]$ where a denotes the minor radius of the discharge chamber, $B_{\theta}(r, t)$ is the poloidal magnetic field, $B_{z}(r, t)$ is the toroidal magnetic field, and 〈 denotes a volume average. The trajectory shows characteristic RFP behavior and closely follows that predicted by the Modified Bessel Function Model.2,17

Spectroscopic observations of round-top discharges indicate that $D_{\alpha}$ emission [Fig. X.1(e)], which is an indicator of gas recycling and hence plasma-wall interaction, is dependent on the local equilibrium control at the gap. Carbon emission, presumably from the 32 graphite ring limiters, is also a function of equilibrium control, with minimum emission correlating with minimum equilibrium displacement. Emission of OIII through oVII impurity lines shcws the proper time sequencing and plasma current dependence to indicate that the oxygen radiation barrier has been surpassed. In addition, the existence of the OVII line even at moderate currents (Fig. X.3) indicates electron temperatures of at least $70 \mathrm{eV}$.

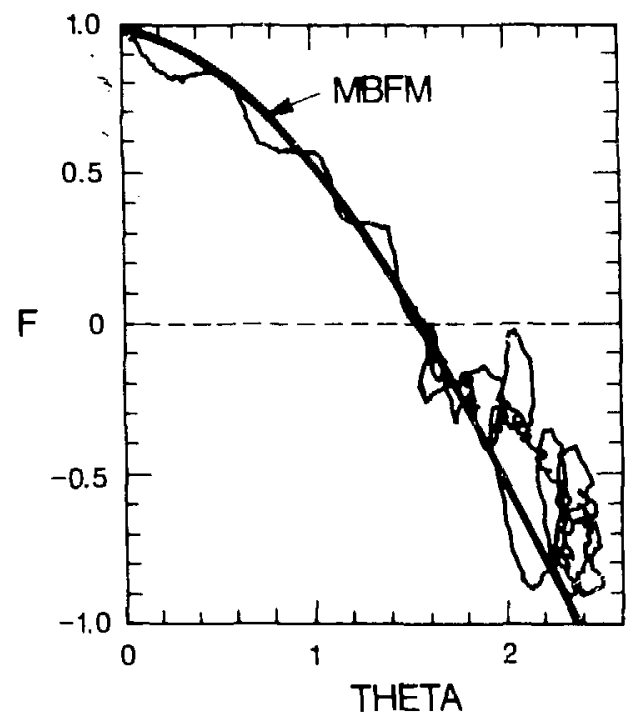

Fig. X. 2 .

F- $\theta$ trajectory for a $60-\mathrm{kA}$ round-top discharge. The bold curve denotes the Modified Bessel Function Model (MBFM) prediction. 


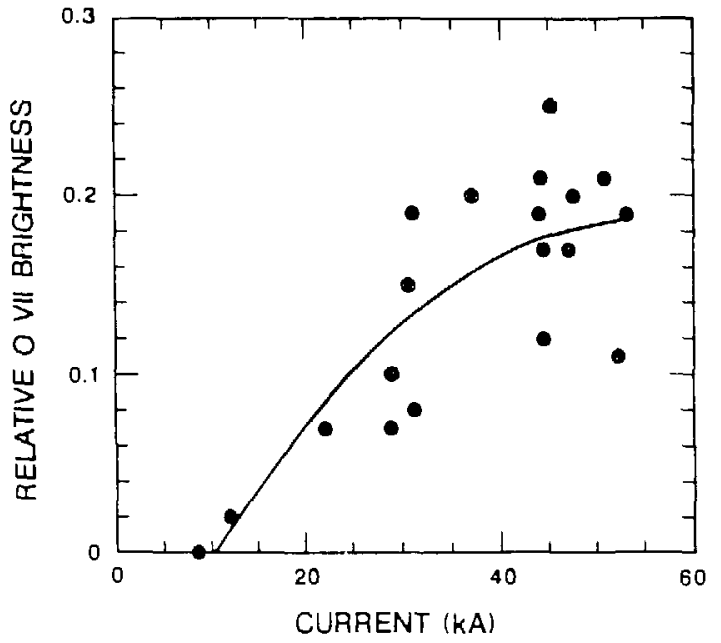

Fig. X. 3 .

Relative OVII brightness versus current for a series of round-top discharges.

The 70-eV or greater electron temperature, inferred spectroscopically, agrees with spatially-averaged conductivity temperature estimates based on plasma resistance assuming a $Z_{\text {eff }}$ of approximately 2.5 . Figure X.4 plots the temporal dependence of plasma resistance. Here, $R_{p}$ is defined in terms of the total power balance (Poynting's Theorem):

$$
R_{p}=\frac{1}{I_{\phi}^{2}}\left\{-\int E \times B \cdot d S-\frac{d W_{M}}{d t}\right\},
$$

where - $\int E \times B \cdot d S$ is the power input to the plasma, $W_{M}$ is the total magnetic energy in the plasma, and $I_{\phi}$ is the toroidal plasma current. 


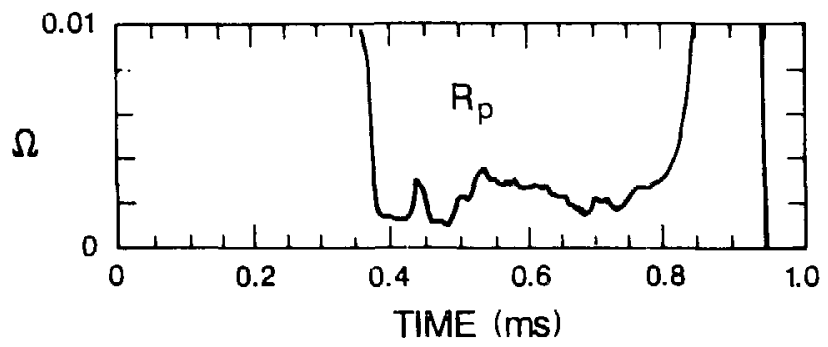

Fig. X. 4 .

Temporal dependence of plasma resistance (based on power balance) for a 60-kA round-top discharge.

\section{Flat Top Discharges}

Figures X.5 through X.7 display data from a 35-kA flat-topped discharge. In this discharge, both the toroidal and poloidal magnetic fields were power crowbarred, yielding a total duration of 1.2 ms with a $0.6-m s$ flat-top. The discharge exhibits the same basic F- $\Theta$ behavior and resistance as seen in comparable current round-top discharges with the addition that all parameters are held constant during the sustainment. Despite the application of power crowbars, the discharge terminates when the local equilibrium at the gap deteriorates, as was the case for round-topped discharges. 

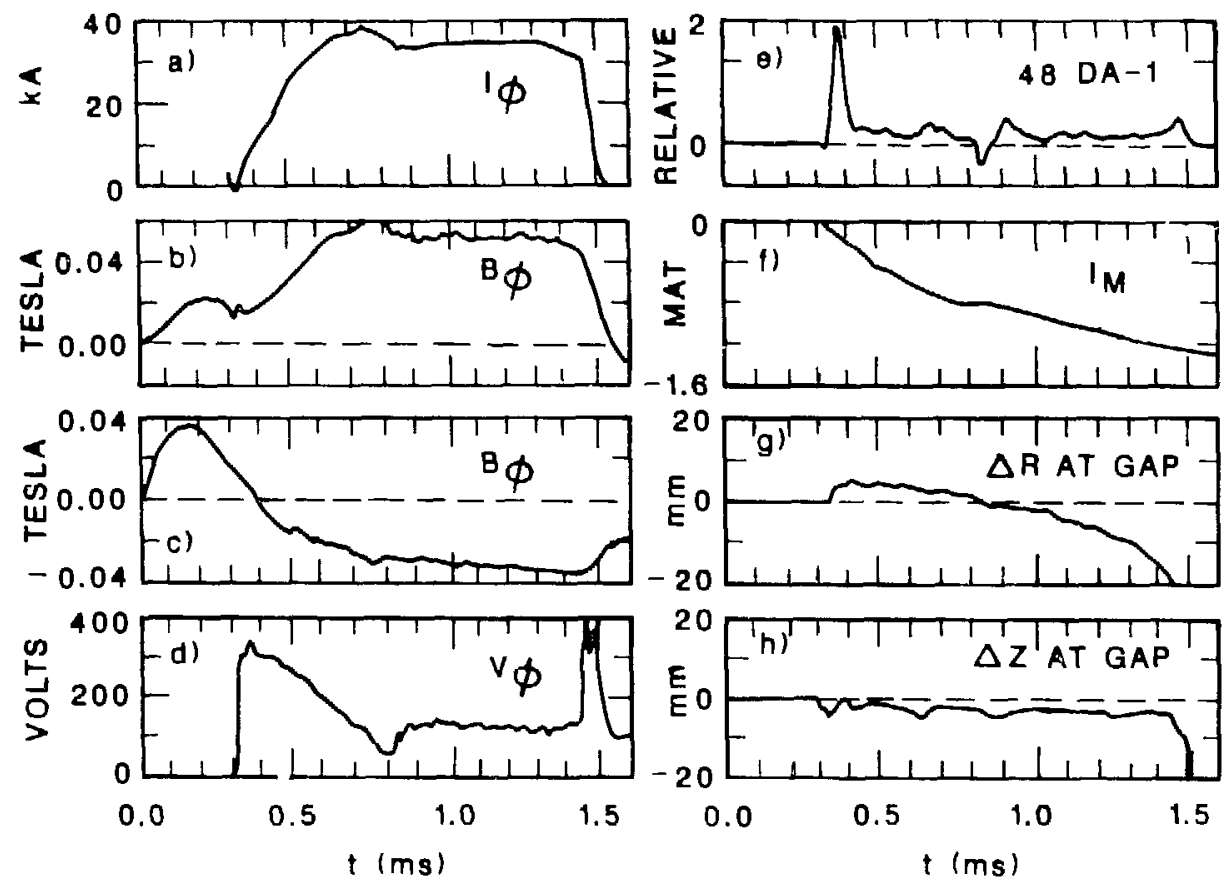

Fig. X.5.

Condensed parameters for a 35-kA flat-topped discharge: (a) Toroidal plasma current, (b) Volume averaged toroidal magnetic field, (c) Toroidal magnetic field at the plasma edge, (d) Toroidal loop voltage, (e) $\mathrm{D}_{\alpha}$ emission, (f) Magnetizing current in MA-turns, (g) Outer flux surface radial displacement at the gap, (h) Vertical displacement at the gap. 


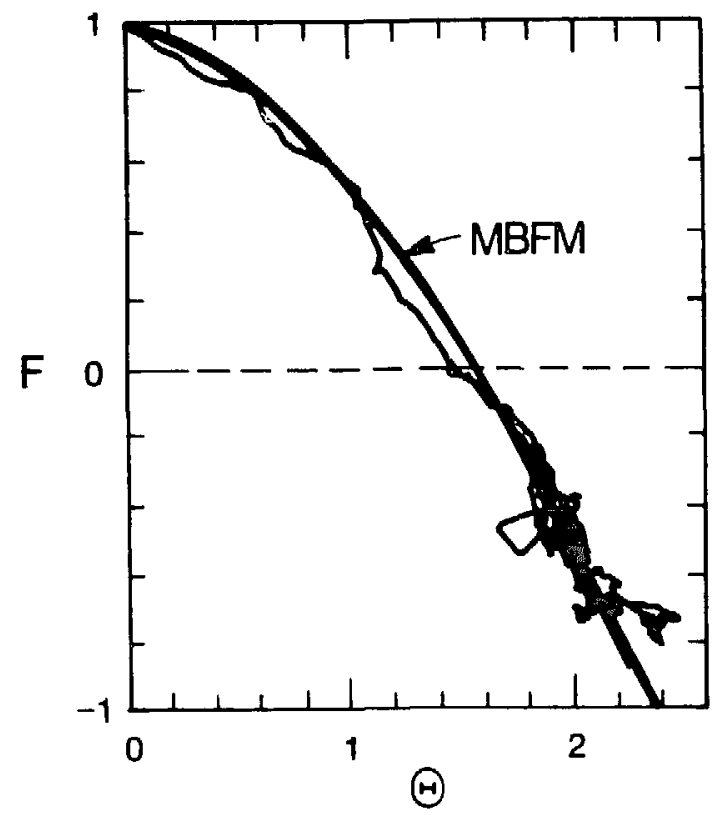

Fig. X.6.

F-0 trajectory for a 35-kA flat-topped discharge. The bold curve denotes the MBFM prediction.

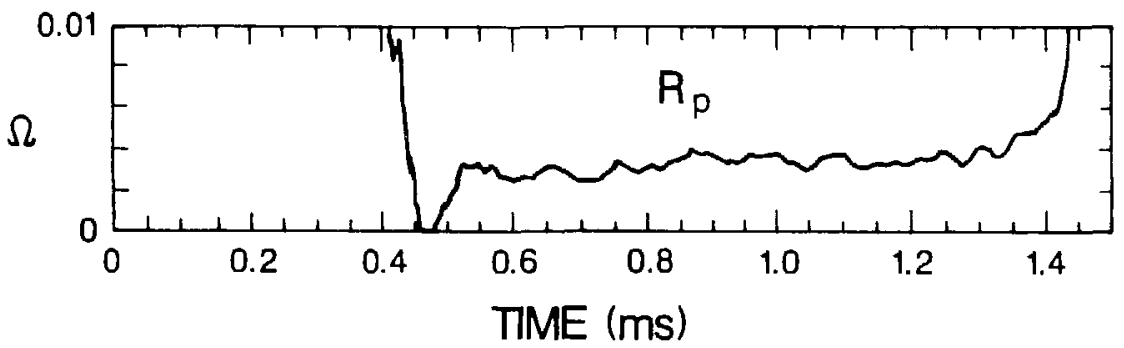

Fig. X.7.

Plasma resistance for a $35-\mathrm{kA}$ flat-topped discharge. 
The effect of equilibrium control on discharge duration is illustrated in Fig. X.8. Here, two discharges were run with all operational parameters (i.e., applied voltage, circuit timing, gas fill pressure, etc.) held fixed except the applied vertical field. In discharge (e), the vertical field was overdriven. This caused the radial equilibrium displacement at the gap [Fig. $X .8(\mathrm{~g})$ ] to move inward (towards the major axis o $\bar{i}$ the torus) after discharge initiation and ultimately resulted in a premature termination. Although the physical mechanism of termination and its quantitative dependence on equilibrium control is not yet understood, it is expected that discharge duration will increase with feedback control of the equilibrium vertical field.
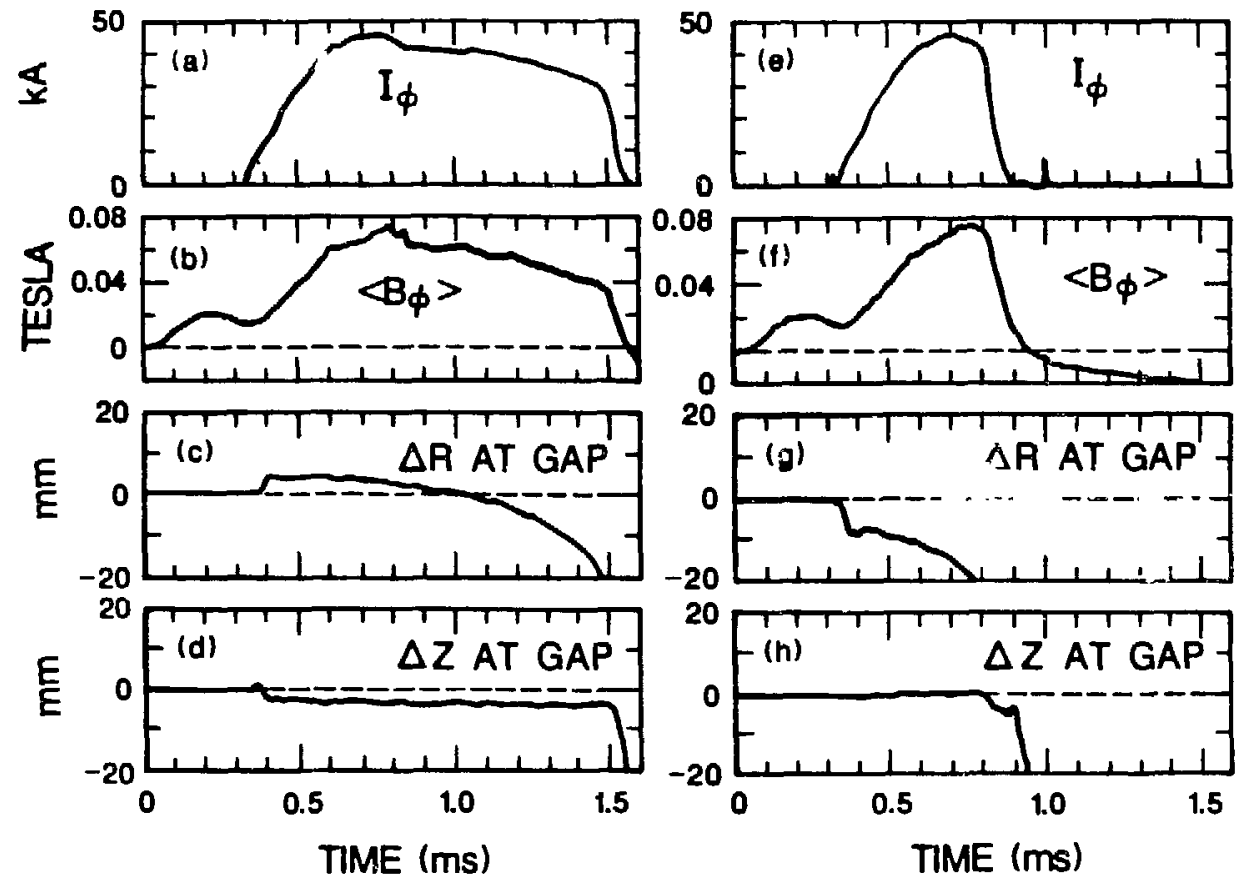

Fig. X. 8 .

Effect of equilibrium position at the gap on termination. All parameters held fixed except (a) through (d) have a smailer vertical field to plasma current ratio than (e) through (h).

\section{ACKNOWLEDGEMENT}

We wish to thank Mabel Marquez for her valuable assistance in the preparation of this manuscript. 


\section{REFERENCES}

1. R. L. Hagensoin and R. A. Krakowski, "Compact Reversed-Field Pinch Reactor (CRFPR) Sensitivity and Design Point Determination," Los Alamos National Laboratory report LA-9389-MS (1982).

2. K. F. Schoenberg, R. F. Gribble, and J. A. Phillips, "Zero-Dimensional Simulations of Reversed Field Pinch Experiments," Nucl. Fusion 22, 1433 (1982).

3. K. F. Schoenberg, R. F. Gribble, and D. A. Baker, J. Appl. Phys. 56 (9), 2521 (1984).

4. D. A. Baker, L. W. Mann, and K. F. Schoenberg, "Equilibrium Poloidal Field Distributions in Axisymmetric RFP Toroidal Discharges," Nucl. Fusion 23, 380 (1982).

5. W. A. Reass, J. G. Melton, and R. F. Gribble, "Proceedings of the 10 th Symposium on Fusion Engineering," Philadelphia, PA, 1512, (1983).

6. J. N. Downing, R. A. Gordon, K. S. Thomas, and R. G. Watt, "Limiter Study," Los Alamos National Laboratory report LA-9774-MS (1983).

7. J. N. Downing, C. J. Buchenauer, J. C. Ingraham, R. A. Krakowski, J. A. Phillips, and R. G. Watt, "An Evaluation of Limiter Configurations in ZT-40M," J. Nucl. Mater. 128 \& 129, 51?-523 (1984).

8. H. W. Moos, K. I. Chen, J. L. Terry, and W. G. Fastie, Appl. Optics 18, 1209 (1979).

9. P. G. Weber, Phys. Fluids 28, 3136 (1985).

10. J. T. Hogan, "The Effects of Low Energy Electron Capture Collisions $\left(\mathrm{H}_{0}+\mathrm{C}^{\mathrm{nt}}\right)$ on the Particle and Energy Balance of Tokamak Plasmas" in Physics of Electronic and Atomic Collisions, S. Datz (Ed.), North Holland Publishing Co., (1982).

11. L. Johnson and E. Hinnov, J. Quant. Spectros. Radiat. Transfer 13, 333 (1973).

12. G. A. Wurden, Phys. Fluids 27(3), 551 (1984).

13. M. Malacarne and I. Hutchinson, "Soft X-Ray Imaging Diagnostic on the HBTX 1A Reversed Field Pinch," Culham Laboratory Report CLM-R227, 1982.

14. D. A. Baker, C. J. Buchenauer, L. C. Burkhardt, J. N. Downing,

K. B. Freese, R. F. Gribble, A. Haberstich, R. B. Howell, A. R. Jacobson,

K. A. Klare, G. Miller, J. A. Phillips, K. S. Thomas, R. G. Watt, and

P. G. Weber, "Four-Feedplate Operation of ZT-40 With Passive Crowbars," Los Alamos National Laboratory report LA-8752-MS (1982). 
15. E. J. Caramana and D. A. Baker, Nucl. Fusion 24, 423 (1984).

16. R. S. Massey, C. J. Buchenauer, L. C. Burkhardt, A. R. Jacobson, J. G. Melton, G. Miller, R. W. Moses, K. F. Schoenberg, and R. G. Wat t, "Preliminary Equilibrium and Field Error Studies on ZT-40M," Los Alamos National Laboratory report LA-9567-MS (February 1983).

17. K. F. Schoenberg, R. W. Moses, Jr., and R. A. Hagenson, Phys. Fluids 27 , 1671 (1984). 